\title{
Synthesis of Substituted Quinolines by Electrophilic Cyclization of $N$-(2-
}

\section{Alkynyl)anilines}

\author{
Xiaoxia Zhang, Marino A. Campo, Tuanli Yao, and Richard C. Larock* \\ Department of Chemistry, Iowa State University, Ames, Iowa 50011
}

\section{Supporting Information}

General. The ${ }^{1} \mathrm{H}$ and ${ }^{13} \mathrm{C}$ NMR spectra were recorded at 300 and $75.5 \mathrm{MHz}$ or 400 and $100 \mathrm{MHz}$ respectively. All melting points are uncorrected. High resolution mass spectra were recorded on a Kratos MS50TC double focusing magnetic sector mass spectrometer using $\mathrm{EI}$ at $70 \mathrm{eV}$. All reagents were used directly as obtained commercially unless otherwise noted.

Synthesis of Starting Materials. Compounds 1, 3, 6, 8, and 22 were prepared by the Sonogashiro reaction of $N$-(2-propynyl)aniline with the corresponding aryl halide.

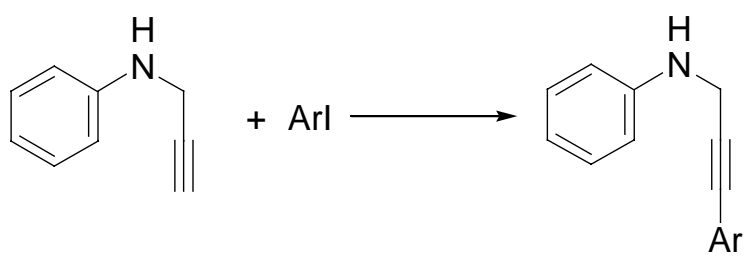

A typical reaction is described below. To a solution of $\mathrm{Et}_{3} \mathrm{~N}(30 \mathrm{~mL}), \mathrm{PdCl}_{2}\left(\mathrm{PPh}_{3}\right)_{2}(2$ mol \%), $3.0 \mathrm{mmol}$ of $\mathrm{N}$-(2-propynyl)aniline, and 1.2 equiv of aryl iodide (stirring for 3 min beforehand) was added $\mathrm{CuI}(1 \mathrm{~mol} \%)$. The reaction mixture was flushed with $\mathrm{Ar}$ and the flask was then sealed. The mixture was stirred at room temperature and was monitored by TLC to establish completion of the reaction. The resulting solution was filtered, washed with a satd aq $\mathrm{NaCl}$ solution, and extracted with diethyl ether. The 
combined ether fractions were dried over $\mathrm{MgSO}_{4}$ and concentrated under vacuum to yield the crude product, which was purified by flash chromatography on silica gel.

$N$-(3-Phenyl-2-propynyl)aniline (1). The indicated compound was prepared in a $75 \%$ yield as a light yellow oil from the coupling of $N$-(2-propynyl)aniline with iodobenzene. The reaction mixture was chromatographed using 5:1 hexane/EtOAc. The spectral properties were identical with those previously reported. ${ }^{1}$

$N$-[3-(4-Methoxyphenyl)prop-2-ynyl]- $N$-phenylamine (3). The indicated compound was prepared as a yellow oil from $p$-iodoanisole in a $70 \%$ yield. The reaction mixture was chromatographed using 5:1 hexane/EtOAc. ${ }^{1} \mathrm{H} \mathrm{NMR}\left(\mathrm{CDCl}_{3}, 400 \mathrm{MHz}\right) \delta$ $3.67(\mathrm{~s}, 3 \mathrm{H}), 3.92(\mathrm{~s}, 1 \mathrm{H}), 4.04(\mathrm{~s}, 2 \mathrm{H}), 6.66(\mathrm{~d}, J=11.6 \mathrm{~Hz}, 2 \mathrm{H}), 6.72-6.76(\mathrm{~m}, 3 \mathrm{H})$, 7.15-7.21 (m, 2H), $7.31(\mathrm{~d}, J=11.6 \mathrm{~Hz}, 2 \mathrm{H}) ;{ }^{13} \mathrm{C} \mathrm{NMR}\left(\mathrm{CDCl}_{3}, 100 \mathrm{MHz}\right) \delta 34.66$, $55.36,83.29,85.28,113.69,114.03,114.08,115.14,118.47,129.41,133.32,147.44$, 159.68; IR $\left(\mathrm{CHCl}_{3}\right)$ 3398, 2929, 2833, 1603, $1504 \mathrm{~cm}^{-1}$; HRMS m/z 237.1157 (calcd for $\left.\mathrm{C}_{16} \mathrm{H}_{15} \mathrm{NO}, 237.1154\right)$.

$N$-[3-(4-Fluorophenyl)prop-2-ynyl]- $N$-phenylamine (6). The indicated compound was prepared as a light orange oil from 4-fluoro-1-iodobenzene in an $81 \%$ yield. The reaction mixture was chromatographed using 5:1 hexane/EtOAc. ${ }^{1} \mathrm{H}$ NMR $\left(\mathrm{CDCl}_{3}, 300 \mathrm{MHz}\right) \delta 3.98(\mathrm{~s}, 1 \mathrm{H}), 4.16(\mathrm{~s}, 2 \mathrm{H}), 6.76(\mathrm{~d}, J=9.3 \mathrm{~Hz}, 2 \mathrm{H}), 6.81-6.85(\mathrm{~m}$, 1H), 6.97-7.03 (m, 2H), 7.24-7.29 (m, 2H), 7.37-7.42 (m, 2H); ${ }^{13} \mathrm{C} \mathrm{NMR}\left(\mathrm{CDCl}_{3}, 75\right.$ MHz) $\delta 34.73,82.48,86.38,86.40,113.83,115.64,115.93,118.78,119.19,119.23$, $129.52,133.80,133.91,147.36,161.05,164.36$ (the extra peaks are due to ${ }^{19} \mathrm{~F}$ splitting); IR $\left(\mathrm{CHCl}_{3}\right) 3407,1605 \mathrm{~cm}^{-1}$; HRMS m/z, 225.0957 (calcd for $\left.\mathrm{C}_{15} \mathrm{H}_{12} \mathrm{FN}, 225.0954\right)$. 
1-[4-(3-Phenylaminoprop-1-ynyl)phenyl]ethanone (8). The indicated compound was prepared as a pale yellow solid from 4-iodoacetophenone in a $78 \%$ yield. The reaction mixture was chromatographed using 3:1 hexane/EtOAc: mp $95-96{ }^{\circ} \mathrm{C} ;{ }^{1} \mathrm{H}$ NMR $\left(\mathrm{CDCl}_{3}, 300 \mathrm{MHz}\right) \delta 2.58(\mathrm{~s}, 3 \mathrm{H}), 3.9-4.1$ (br s, 1H), $4.18(\mathrm{~s}, 2 \mathrm{H}), 6.73-6.83(\mathrm{~m}$, $3 \mathrm{H}), 7.21-7.27(\mathrm{~m}, 2 \mathrm{H}), 7.47(\mathrm{~d}, J=6.9 \mathrm{~Hz}, 2 \mathrm{H}), 7.87(\mathrm{~d}, J=6.6 \mathrm{~Hz}, 2 \mathrm{H}) ;{ }^{13} \mathrm{C} \mathrm{NMR}$ $\left(\mathrm{CDCl}_{3}, 75 \mathrm{MHz}\right) \delta 26.85,34.80,82.79,90.17,113.83,118.90,128.01,128.40,129.51$, 132.07, 136.48, 147.15, 197.54; IR $\left(\mathrm{CHCl}_{3}\right) 3375,2826,1676,1602,1513 \mathrm{~cm}^{-1}$; HRMS $m / z$ 249.1156 (calcd for $\mathrm{C}_{17} \mathrm{H}_{15} \mathrm{NO}, 249.1154$ ).

2-(3-Phenylaminoprop-1-ynyl)aniline (22). The indicated compound was prepared as a light red oil from 2-iodoaniline in a $45 \%$ yield. The reaction mixture was chromatographed using 3:1 hexane/EtOAc. ${ }^{1} \mathrm{H} \mathrm{NMR}\left(\mathrm{CDCl}_{3}, 300 \mathrm{MHz}\right) \delta 3.96(\mathrm{~s}, 3 \mathrm{H})$, $4.14(\mathrm{~s}, 2 \mathrm{H}), 6.56-6.70(\mathrm{~m}, 2 \mathrm{H}), 6.71-6.80(\mathrm{~m}, 3 \mathrm{H}), 7.02-7.08(\mathrm{~m}, 1 \mathrm{H}), 7.10-7.19(\mathrm{~m}$, 1H), 7.21-7.23 (m, 2H); ${ }^{13} \mathrm{C} \mathrm{NMR}\left(\mathrm{CDCl}_{3}, 75 \mathrm{MHz}\right) \delta 35.03,80.32,92.18,114.20$, $114.55,115.40,118.01,118.94,129.58,129.89,132.32,147.38,148.40 ; \mathrm{IR}\left(\mathrm{CHCl}_{3}\right)$ 3374, 3051, 2848 1602, $1500 \mathrm{~cm}^{-1}$; HRMS m/z 222.1161 (calcd for $\mathrm{C}_{15} \mathrm{H}_{14} \mathrm{~N}_{2}, 222.1157$ ).

Compounds $10,12,17$, and 20 were prepared by the reaction of the appropriate aniline ( 2 equiv) and the corresponding propargylic mesylate at room temperature in $\mathrm{CH}_{3} \mathrm{CN}^{2}$

$N$-(2-Heptynyl)aniline (10). To a solution of the methanesulfonate ${ }^{3}$ of 2-heptyn1-ol (950 mg, $5.0 \mathrm{mmol})$ in $50 \mathrm{~mL}$ of $\mathrm{CH}_{3} \mathrm{CN}$ was added aniline (930 mg, $10 \mathrm{mmol}$ ). After being stirred for $20 \mathrm{~h}$ under $\mathrm{N}_{2}$, the reaction was quenched by adding brine. The reaction mixture was extracted with $\mathrm{Et}_{2} \mathrm{O}(2 \times 30 \mathrm{~mL})$. The extracts were dried over $\mathrm{MgSO}_{4}$ and the solvent was removed under reduced pressure. The residue was purified 
by flash chromatography (10:1 hexane/EtOAc) on silica gel to afford $589 \mathrm{mg}$ of the product in a $63 \%$ yield as a light yellow oil. The spectra properties were identical with those previously reported. ${ }^{4}$

$N$-(3-Cyclohex-1-en-1-yl-1-methylprop-2-ynyl)aniline (12). Using the procedure used to prepare aniline 10, aniline and the methanesulfonate of 4-(1cyclohexen-1-yl)-3-butyn-2-ol were employed to afford the indicated compound in a 35 $\%$ yield as a colorless oil. The reaction mixture was chromatographed using 10:1 hexane/EtOAc. ${ }^{1} \mathrm{H}$ NMR $\left(\mathrm{CDCl}_{3}, 300 \mathrm{MHz}\right) \delta 1.47-1.58(\mathrm{~m}, 7 \mathrm{H}), 2.00-2.03(\mathrm{~m}, 4 \mathrm{H})$, $3.69(\mathrm{~s}, 1 \mathrm{H}), 4.28-4.30(\mathrm{q}, J=6.6 \mathrm{~Hz}, 1 \mathrm{H}), 5.99-6.01(\mathrm{~m}, 1 \mathrm{H}), 6.65-6.74(\mathrm{~m}, 3 \mathrm{H}), 7.14-$ $7.19(\mathrm{~m} 2 \mathrm{H}) ;{ }^{13} \mathrm{C} \mathrm{NMR}\left(\mathrm{CDCl}_{3}, 75 \mathrm{MHz}\right) \delta 21.82,22.61,22.92,25.88,29.60,41.71$, 84.19, 88.55, 114.32, 118.42, 120.67, 129.39, 134.83, 147.05; IR $\left(\mathrm{CHCl}_{3}\right) 3397,2933$, $1605,1505 \mathrm{~cm}^{-1}$; HRMS m/z 225.1520 (calcd for $\mathrm{C}_{16} \mathrm{H}_{19} \mathrm{~N}, 225.1518$ ).

$N$-(3-Phenylprop-2-ynyl)-3-nitroaniline (17). Using the procedure used to prepare aniline 10, 3-nitroaniline and the methanesulfonate of 3-phenyl-2-propyn-1-ol were employed to afford the indicated compound in a $55 \%$ yield as an orange oil. The reaction mixture was chromatographed using 10:1 hexane/EtOAc. ${ }^{1} \mathrm{H}$ NMR $\left(\mathrm{CDCl}_{3}, 400\right.$ MHz) $\delta 4.20$ (s, 2H), 4.50-5.50 (br s, 1H), 6.96-7.00 (m, 1H), 7.25-7.40 (m, 6H), 7.54$7.61(\mathrm{~m}, 2 \mathrm{H}) ;{ }^{13} \mathrm{C} \mathrm{NMR}\left(\mathrm{CDCl}_{3}, 100 \mathrm{MHz}\right) \delta 34.33,84.05,84.97,107.39,113.04$, 119.53, 122.48, 128.40, 128.57, 129.82, 131.80; IR $\left(\mathrm{CHCl}_{3}\right)$ 3060, 1686, 1622, 1528, $1348 \mathrm{~cm}^{-1}$; HRMS $m / z 252.0902$ (calcd for $\mathrm{C}_{15} \mathrm{H}_{12} \mathrm{~N}_{2} \mathrm{O}_{2}, 252.0899$ ).

$N$-(2-Naphthyl)- $N$-(3-phenylprop-2-ynyl)amine (20). Using the procedure used to prepare aniline 10,2-aminonaphthalene and the methanesulfonate of 3-phenyl-2propyn-1-ol were employed to afford the indicated compound in a $50 \%$ yield as an light 
red oil. The reaction mixture was chromatographed using 10:1 hexane/EtOAc. ${ }^{1} \mathrm{H}$ NMR $\left(\mathrm{CDCl}_{3}, 300 \mathrm{MHz}\right) \delta 4.02(\mathrm{~s}, 1 \mathrm{H}), 4.13(\mathrm{~s}, 2 \mathrm{H}), 6.82-6.91(\mathrm{~m}, 2 \mathrm{H}), 7.18-7.24(\mathrm{~m}, 4 \mathrm{H})$, 7.33-7.40 (m, 3H), 7.59-7.67 (m, 3H); ${ }^{13} \mathrm{C} \mathrm{NMR}\left(\mathrm{CDCl}_{3}, 75 \mathrm{MHz}\right) \delta 34.86,83.83,86.68$, $106.05,118.45,122.83,123.22,126.61,126.75,128.06,128.32,128.65,128.67,129.33$, 132.10, 135.37, 145.14; IR ( $\left.\mathrm{CHCl}_{3}\right) 3409,3054,1632,1604,1519,1488 \mathrm{~cm}^{-1}$; HRMS $m / z, 257.1204$ (calcd for $\mathrm{C}_{19} \mathrm{H}_{15} \mathrm{~N}, 257.1209$ ).

Ethyl 4-[(3-phenylprop-2-ynyl)amino]benzoate (14). To a solution of ethyl 4aminobenzoate $(639 \mathrm{mg}, 3.0 \mathrm{mmol})$ and $\mathrm{K}_{2} \mathrm{CO}_{3}(621 \mathrm{mg}, 4.5 \mathrm{mmol})$ in $30 \mathrm{~mL}$ of DMF was added 3-bromo-1-phenylpropyne $(1.17 \mathrm{~g}, 6.0 \mathrm{mmol})$. The resulting solution was heated at $80{ }^{\circ} \mathrm{C}$ for $24 \mathrm{~h}$. The reaction mixture was quenched with brine and extracted with ethyl acetate. The organic extracts were washed with brine to remove the DMF. The solvent was removed under reduced pressure and the residue was chromatographed using 3:1 hexane/EtOAc. The indicated compound was obtained as a light yellow solid in a $56 \%$ yield: $\mathrm{mp} 104-105{ }^{\circ} \mathrm{C} ;{ }^{1} \mathrm{H} \mathrm{NMR}\left(\mathrm{CDCl}_{3}, 300 \mathrm{MHz}\right) \delta 1.34-1.39(\mathrm{t}, J=7.2 \mathrm{~Hz}$, $3 \mathrm{H}), 4.19(\mathrm{~s}, 2 \mathrm{H}), 4.32-4.36(\mathrm{q}, J=7.2 \mathrm{~Hz}, 2 \mathrm{H}), 4.46(\mathrm{~s}, 1 \mathrm{H}), 6.68(\mathrm{~d}, J=8.7 \mathrm{~Hz}, 2 \mathrm{H})$, 7.26-7.31 (m, 3H), 7.38-7.41 (m, 2H), $7.93(\mathrm{~d}, J=6.9 \mathrm{~Hz}, 2 \mathrm{H}) ;{ }^{13} \mathrm{C} \mathrm{NMR}\left(\mathrm{CDCl}_{3}, 75\right.$ MHz) $\delta 14.68,34.22,60.55,83.91,85.53,112.43,120.08,122.80,128.54,128.65$, 131.65, 131.95, 151.02, 167.05; IR $\left(\mathrm{CHCl}_{3}\right) 3372,2982,1693,1602,1527,1280 \mathrm{~cm}^{-1}$; HRMS $m / z 279.1263$ (calcd for $\mathrm{C}_{18} \mathrm{H}_{11} \mathrm{NO}_{2}, 279.1259$ ).

General Procedure for the Electrophilic Cyclization of $N$-(2-Alkynyl)anilines by $\mathbf{I}_{2 .} 0.3 \mathrm{Mmol}$ of the propargylic aniline, 3 equiv of $\mathrm{I}_{2}, 2$ equiv of $\mathrm{NaHCO}_{3}$, and $3 \mathrm{~mL}$ of $\mathrm{CH}_{3} \mathrm{CN}$ were placed in a vial. The reaction mixture was stirred at room temperature, and the reaction was monitored by TLC to establish completion. When finely ground 
iodine powder was employed, all reactions were complete in $0.5 \mathrm{~h}$. The reaction mixture was then diluted with $25 \mathrm{~mL}$ of ether, and washed with $20 \mathrm{~mL}$ of satd aq $\mathrm{Na}_{2} \mathrm{~S}_{2} \mathrm{O}_{3}$. The organic layer was separated and the aqueous layer was extracted with another $25 \mathrm{~mL}$ of ether. The combined organic layers were dried over $\mathrm{MgSO}_{4}$ and filtered. The solvent was evaporated under reduced pressure and the product was isolated by chromatography on a silica gel column.

\section{General Procedure for the Electrophilic Cyclization of $N$-(2-Alkynyl)anilines}

by ICl and PhSeBr. $0.3 \mathrm{Mmol}$ of the propargylic aniline, 2 equiv of $\mathrm{NaHCO}_{3}$ and $2 \mathrm{~mL}$ of $\mathrm{CH}_{3} \mathrm{CN}$ were placed in a vial. 2 Equiv of $\mathrm{ICl}$ or $\mathrm{PhSeBr}$ in $1 \mathrm{~mL}$ of $\mathrm{CH}_{3} \mathrm{CN}$ were added dropwise to the vial. The reaction mixture was stirred at room temperature for 5 min. The reaction mixture was then diluted with $25 \mathrm{~mL}$ of ether, and washed with $20 \mathrm{~mL}$ of satd aq $\mathrm{Na}_{2} \mathrm{~S}_{2} \mathrm{O}_{3}$ (for the reaction of $\mathrm{ICl}$ ) or satd aq $\mathrm{NaCl}$ (for the reaction of $\mathrm{PhSeBr}$ ). The organic layer was separated and the aqueous layer was extracted with another $25 \mathrm{~mL}$ of ether. The combined organic layers were dried over $\mathrm{MgSO}_{4}$ and filtered. The solvent was evaporated under reduced pressure and the product was isolated by chromatography on a silica gel column.

3-Iodo-4-phenylquinoline (2). The reaction mixture was chromatographed using 5:1 hexane/EtOAc to afford $75 \mathrm{mg}(76 \%)$ or $82 \mathrm{mg}(83 \%)$ of the product as a white solid from $\mathrm{I}_{2}$ or $\mathrm{ICl}$, respectively: $\mathrm{mp} 131-132{ }^{\circ} \mathrm{C} ;{ }^{1} \mathrm{H} \mathrm{NMR}\left(\mathrm{CDCl}_{3}\right) \delta$ 7.25-7.28 (m, 2H), 7.42-7.48 (m, 2H), 7.52-7.55 (m, 3H), 7.69-7.74 (m, 1H), 8.12 (d, J = 8.8 Hz, 1H), 9.24 $(\mathrm{s}, 1 \mathrm{H}) ;{ }^{13} \mathrm{C} \mathrm{NMR}\left(\mathrm{CDCl}_{3}\right) \delta 96.4,126.8,127.4,128.7,129.0,129.1,129.5,140.4,147.2$, 152.4, 156.6 (one $\mathrm{sp}^{2}$ carbon missing due to overlap); IR $\left(\mathrm{CHCl}_{3}\right) 3061,2918,1566$, 1501, $1485 \mathrm{~cm}^{-1}$; HRMS m/z 330.9864 (calcd for $\mathrm{C}_{15} \mathrm{H}_{10} \mathrm{IN}, 330.9858$ ). 
3-Iodo-4-(4-methoxyphenyl)quinoline (4). The reaction mixture was chromatographed using 5:1 hexane/EtOAc to afford $77 \mathrm{mg}(71 \%)$ or $81 \mathrm{mg}(73 \%)$ of the product as a light yellow solid from $\mathrm{I}_{2}$ or $\mathrm{ICl}$, respectively: mp $127-128{ }^{\circ} \mathrm{C} ;{ }^{1} \mathrm{H}$ NMR $\left(\mathrm{CDCl}_{3}, 300 \mathrm{MHz}\right) \delta 3.92(\mathrm{~s}, 3 \mathrm{H}), 7.08(\mathrm{~d}, J=8.7 \mathrm{~Hz}, 2 \mathrm{H}), 7.21(\mathrm{~d}, J=8.7 \mathrm{~Hz}, 2 \mathrm{H}), 7.42-$ $7.45(\mathrm{~m}, 1 \mathrm{H}), 7.52(\mathrm{~d}, J=8.4 \mathrm{~Hz}, 1 \mathrm{H}), 7.68-7.73(\mathrm{~m}, 1 \mathrm{H}), 8.11(\mathrm{~d}, J=8.4 \mathrm{~Hz}, 1 \mathrm{H}), 9.23$ $(\mathrm{s}, 1 \mathrm{H}) ;{ }^{13} \mathrm{C} \mathrm{NMR}\left(\mathrm{CDCl}_{3}, 75 \mathrm{MHz}\right) \delta 55.58,97.29,114.22,127.03,127.52,129.54$, 129.70, 129.85, 130.66, 132.80, 147.49, 152.40, 156.87, 159.99; IR $\left(\mathrm{CHCl}_{3}\right) 2837,1613$, 1513, 1483, $1246 \mathrm{~cm}^{-1}$; HRMS m/z 360.9972 (calcd for $\mathrm{C}_{16} \mathrm{H}_{12} \mathrm{INO}, 360.9964$ ).

4-(4-Methoxyphenyl)-3-(phenylseleno)quinoline (5). The reaction mixture was chromatographed using 3:1 hexane/EtOAc to afford $86 \mathrm{mg}(74 \%)$ of the product as a yellow solid: $\mathrm{mp} 93-94{ }^{\circ} \mathrm{C} ;{ }^{1} \mathrm{H} \mathrm{NMR}\left(\mathrm{CDCl}_{3}, 300 \mathrm{MHz}\right) \delta 3.91(\mathrm{~s}, 3 \mathrm{H}), 7.06(\mathrm{~d}, J=6.0$ $\mathrm{Hz}, 2 \mathrm{H}), 7.26-7.32(\mathrm{~m}, 5 \mathrm{H}), 7.43-7.57(\mathrm{~m}, 3 \mathrm{H}), 7.63-7.68(\mathrm{~m}, 1 \mathrm{H}), 7.68-7.73(\mathrm{~m}, 1 \mathrm{H})$, $8.05(\mathrm{~d}, J=8.4 \mathrm{~Hz}, 1 \mathrm{H}), 8.67(\mathrm{~s}, 1 \mathrm{H}) ;{ }^{13} \mathrm{C} \mathrm{NMR}\left(\mathrm{CDCl}_{3}, 75 \mathrm{MHz}\right) \delta 55.57,114.25$, $126.23,127.22,127.34,128.44,128.47,129.16,129.61,129.70,129.74,129.85,130.87$, 134.81, 147.08, 148.27, 152.43, 159.99; IR $\left(\mathrm{CHCl}_{3}\right)$ 2923, 2851, 1736, $1250 \mathrm{~cm}^{-1}$; HRMS m/z 391.0482 (calcd for $\mathrm{C}_{22} \mathrm{H}_{17} \mathrm{NOSe}, 391.0475$ ).

4-(4-Fluorophenyl)-3-iodoquinoline (7). The reaction mixture was chromatographed using 5:1 hexane/EtOAc to afford $82 \mathrm{mg}(78 \%)$ of the product as a white solid: $\mathrm{mp} 108-109{ }^{\circ} \mathrm{C} ;{ }^{1} \mathrm{H}$ NMR $\left(\mathrm{CDCl}_{3}, 300 \mathrm{MHz}\right) \delta$ 7.24-7.26 (m, 4H), 7.44-7.45 $(\mathrm{m}, 2 \mathrm{H}), 7.70-7.76(\mathrm{~m}, 1 \mathrm{H}), 8.12(\mathrm{~d}, J=8.4 \mathrm{~Hz}, 1 \mathrm{H}), 9.24(\mathrm{~s}, 1 \mathrm{H}) ;{ }^{13} \mathrm{C} \mathrm{NMR}\left(\mathrm{CDCl}_{3}, 75\right.$ MHz) $\delta 96.85,96.86,115.93,116.22,126.65,127.78,129.21,129.81,130.03,131.16$, 131.27, 136.39, 136.43, 147.41, 151.55, 156.82, 161.41, 164.70; IR $\left(\mathrm{CHCl}_{3}\right) 3067,1617$ 1513, $1494 \mathrm{~cm}^{-1}$; HRMS m/z 348.9770 (calcd for $\mathrm{C}_{15} \mathrm{H}_{9} \mathrm{FIN}, 348.9764$ ). 
1-[4-(3-Iodoquinolin-4-yl)phenyl]ethanone (9). The reaction mixture was chromatographed using 3:1 hexane/EtOAc to afford $64 \mathrm{mg}(57 \%)$ of the product as a yellow solid: $\mathrm{mp} 136-137{ }^{\circ} \mathrm{C} ;{ }^{1} \mathrm{H} \mathrm{NMR}\left(\mathrm{CDCl}_{3}, 300 \mathrm{MHz}\right) \delta 2.72(\mathrm{~s}, 3 \mathrm{H}), 7.37-7.47$ (m, 4H), 7.72-7.78 (m, 1H), 8.13-8.18 (m, 3H), $9.26(\mathrm{~s}, 1 \mathrm{H}) ;{ }^{13} \mathrm{C} \mathrm{NMR}\left(\mathrm{CDCl}_{3}, 75 \mathrm{MHz}\right) \delta$ $26.83,95.51,126.25,127.75,128.47,128.78,129.58,129.71,130.00,137.17,144.97$, 147.12, 151.23, 156.59, 197.62; IR $\left(\mathrm{CHCl}_{3}\right) 3067,1683,1268 \mathrm{~cm}^{-1} ; \mathrm{HRMS} \mathrm{m} / z 372.9970$ (calcd for $\mathrm{C}_{17} \mathrm{H}_{12} \mathrm{INO}, 372.9964$ ).

4-Butyl-3-iodoquinoline (11). The reaction mixture was chromatographed using 10:1 hexane/EtOAc to afford $40 \mathrm{mg}(43 \%)$ of the product as a colorless oil: ${ }^{1} \mathrm{H}$ NMR $\left(\mathrm{CDCl}_{3}, 300 \mathrm{MHz}\right) \delta 1.00-1.05(\mathrm{t}, J=7.2 \mathrm{~Hz}, 3 \mathrm{H}), 1.55-1.67(\mathrm{~m}, 4 \mathrm{H}), 3.20-3.26(\mathrm{~m}, 2 \mathrm{H})$, 7.54-7.59 (m, 1H), 7.69-7.74 (m, 1H), 8.03-8.08 (m, 2H), 9.09 (s, 1H); ${ }^{13} \mathrm{C} \mathrm{NMR}\left(\mathrm{CDCl}_{3}\right.$, $75 \mathrm{MHz}) \delta 14.14,23.32,31.65,37.01,97.61,124.23,127.43,128.60,129.62,130.42$, 147.63, 151.41, 157.32; IR $\left(\mathrm{CHCl}_{3}\right) 2956,2926,2870,1505 \mathrm{~cm}^{-1} ; \mathrm{HRMS} \mathrm{m/z} 311.0177$ (calcd for $\mathrm{C}_{13} \mathrm{H}_{14} \mathrm{IN}, 311.0171$ ).

4-(Cyclohex-1-enyl)-3-iodo-2-methylquinoline (13). The reaction mixture was chromatographed using 10:1 hexane/EtOAc to afford $84 \mathrm{mg}(80 \%)$ of the product as a white solid: $\mathrm{mp} 99-100{ }^{\circ} \mathrm{C} ;{ }^{1} \mathrm{H} \mathrm{NMR}\left(\mathrm{CDCl}_{3}, 400 \mathrm{MHz}\right) \delta 1.78-1.93(\mathrm{~m}, 4 \mathrm{H}), 2.00-2.14$ (m, 1H), 2.30-2.34 (m, 3H), $2.98(\mathrm{~s}, 3 \mathrm{H}), 5.63-5.65(\mathrm{~m}, 1 \mathrm{H}), 7.43-7.47(\mathrm{~m}, 1 \mathrm{H}), 7.66-$ $7.70(\mathrm{~m}, 1 \mathrm{H}), 7.86(\mathrm{~d}, J=8.4 \mathrm{~Hz}, 1 \mathrm{H}), 7.98(\mathrm{~d}, J=8.4 \mathrm{~Hz}, 1 \mathrm{H}) ;{ }^{13} \mathrm{C} \mathrm{NMR}\left(\mathrm{CDCl}_{3}, 100\right.$ MHz) $\delta 21.97,22.77,25.25,28.78,31.41,99.43,125.98,126.27,126.33,128.66,128.80$ 129.67, 139.34, 146.99, 155.78, 159.92; IR $\left(\mathrm{CHCl}_{3}\right)$ 2927, 1559, 1490, $1436 \mathrm{~cm}^{-1}$; HRMS $m / z 349.0334$ (calcd for $\mathrm{C}_{16} \mathrm{H}_{16} \mathrm{IN}, 349.0327$ ). 
Ethyl 3-iodo-4-phenylquinoline-6-carboxylate (15). The reaction mixture was chromatographed using 1:1 hexane/EtOAc to afford $106 \mathrm{mg}(88 \%)$ of the product as a yellow solid: $\mathrm{mp} 150-151{ }^{\circ} \mathrm{C} ;{ }^{1} \mathrm{H} \mathrm{NMR}\left(\mathrm{CDCl}_{3}, 400 \mathrm{MHz}\right) \delta 1.33-1.36(\mathrm{t}, J=7.2 \mathrm{~Hz}$, $3 \mathrm{H}), 4.32-4.37(\mathrm{q}, J=7.2 \mathrm{~Hz}, 2 \mathrm{H}), 7.26-7.29(\mathrm{~m}, 2 \mathrm{H}), 7.56-7.28(\mathrm{~m}, 3 \mathrm{H}), 8.14(\mathrm{~d}, J=8.8$

$\mathrm{Hz}, 1 \mathrm{H}), 8.22(\mathrm{~m}, 1 \mathrm{H}), 8.28-8.31(\mathrm{~m}, 1 \mathrm{H}), 9.30(\mathrm{~s}, 1 \mathrm{H}) ;{ }^{13} \mathrm{C} \mathrm{NMR}\left(\mathrm{CDCl}_{3}, 100 \mathrm{MHz}\right) \delta$ $14.28,61.46,97.28,128.22,128.83,129.08,129.23,129.26,129.71,129.84,139.61$, 148.97, 153.67, 158.64, 165.82; IR $\left(\mathrm{CHCl}_{3}\right)$ 2974, $1724 \mathrm{~cm}^{-1} ; \mathrm{HRMS} \mathrm{m/z} 403.0075$ (calcd for $\mathrm{C}_{18} \mathrm{H}_{14} \mathrm{INO}_{2}, 403.0069$ ).

Ethyl 4-phenyl-3-(phenylseleno)quinoline-6-carboxylate (16). The reaction mixture was chromatographed using 1:1 hexane/EtOAc to afford $67 \mathrm{mg}$ (56 \%) of the product as a yellow solid: $\mathrm{mp} 125-127{ }^{\circ} \mathrm{C} ;{ }^{1} \mathrm{H} \mathrm{NMR}\left(\mathrm{CDCl}_{3}, 400 \mathrm{MHz}\right) \delta 1.33-1.37(\mathrm{~m}, J$ $=7.2 \mathrm{~Hz}, 3 \mathrm{H}), 4.32-4.36(\mathrm{~m}, J=7.2 \mathrm{~Hz}, 2 \mathrm{H}), 7.26-7.36(\mathrm{~m}, 5 \mathrm{H}), 7.50-7.57(\mathrm{~m}, 5 \mathrm{H})$, 8.08-8.10 (m, 1H), 8.22-8.26 (m, 2H), $8.70(\mathrm{~s}, 1 \mathrm{H}) ;{ }^{13} \mathrm{C} \mathrm{NMR}\left(\mathrm{CDCl}_{3}, 100 \mathrm{MHz}\right) \delta$ $14.29,61.36,127.22,128.23,128.46,128.63,128.78,128.87,128.95,129.01,129.04$, $129.34,129.82,135.07,136.48,148.50,149.01,153.81,166.14$ (one $\mathrm{sp}^{2}$ carbon missing due to overlap); IR $\left(\mathrm{CHCl}_{3}\right)$ 2974, $1721 \mathrm{~cm}^{-1}$; HRMS m/z 433.0589 (calcd for $\left.\mathrm{C}_{24} \mathrm{H}_{19} \mathrm{NOSe}, 433.0581\right)$.

\section{3-Iodo-7-nitro-4-phenylquinoline (18) and 3-iodo-5-nitro-4-phenylquinoline}

(19). The reaction mixture was chromatographed using 3:1 hexane/EtOAc to afford $9 \mathrm{mg}$ of $18(8 \%)$ and $80 \mathrm{mg}$ of $\mathbf{1 9}(71 \%)$. Quinoline 18 was obtained as a light yellow solid: mp 157-158 ${ }^{\circ} \mathrm{C} ;{ }^{1} \mathrm{H}$ NMR $\left(\mathrm{CDCl}_{3}, 400 \mathrm{MHz}\right) \delta$ 7.26-7.28 (m, 2H), 7.58-7.64 (m, 4H), 8.16-8.19 (m, 1H), $9.02(\mathrm{~m}, 1 \mathrm{H}), 9.41(\mathrm{~s}, 1 \mathrm{H}) ;{ }^{13} \mathrm{C} \mathrm{NMR}\left(\mathrm{CDCl}_{3}, 100 \mathrm{MHz}\right) \delta 100.47$, $120.91,125.86,128.65,128.87,129.07,131.77,139.30,146.50,147.98,159.29$ (one sp ${ }^{2}$ 
carbon missing due to overlap); IR $\left(\mathrm{CHCl}_{3}\right) 2930,1544,1352 \mathrm{~cm}^{-1}$; HRMS m/z 375.9715 (calcd $\mathrm{C}_{15} \mathrm{H}_{9} \mathrm{IN}_{2} \mathrm{O}_{2}, 375.9709$ ). Quinoline 19 was obtained as a light yellow solid: $\mathrm{mp}$ $157-158{ }^{\circ} \mathrm{C} ;{ }^{1} \mathrm{H}$ NMR $\left(\mathrm{CDCl}_{3}, 400 \mathrm{MHz}\right) \delta$ 7.16-7.19 (m, 2H), 7.41-7.48 (m, 3H), 7.67$7.69(\mathrm{~m}, 1 \mathrm{H}), 7.73-7.77(\mathrm{~m}, 1 \mathrm{H}), 8.29-8.32(\mathrm{~m}, 1 \mathrm{H}), 9.36(\mathrm{~s}, 1 \mathrm{H}) ;{ }^{13} \mathrm{C} \mathrm{NMR}\left(\mathrm{CDCl}_{3}, 100\right.$ MHz) $\delta 102.44,120.09,123.86,128.22,128.27,129.46,134.13,139.42,148.00,148.20$, 148.97, 158.56; IR $\left(\mathrm{CHCl}_{3}\right)$ 2922, 2855, $1527 \mathrm{~cm}^{-1}$; HRMS m/z 375.9715 (calcd for $\left.\mathrm{C}_{15} \mathrm{H}_{9} \mathrm{IN}_{2} \mathrm{O}_{2}, 375.9709\right)$.

3-Iodo-4-phenylbenzo[g]quinoline (21). The reaction mixture was chromatographed using 3:1 hexane/EtOAc to afford $86 \mathrm{mg}(75 \%)$ as a white solid: $\mathrm{mp}$ 217-219 ${ }^{\circ} \mathrm{C} ;{ }^{1} \mathrm{H}$ NMR $\left(\mathrm{CDCl}_{3}, 300 \mathrm{MHz}\right) \delta$ 7.13-7.16 (m, $\left.1 \mathrm{H}\right)$, 7.23-7.26 (m, 2H), 7.38 (d, $J=8.7 \mathrm{~Hz}, 1 \mathrm{H}), 7.44-7.47(\mathrm{~m}, 1 \mathrm{H}), 7.58-7.61(\mathrm{~m}, 3 \mathrm{H}), 7.85(\mathrm{~d}, J=9.0 \mathrm{~Hz}, 1 \mathrm{H}), 7.99$ (s, 1H), $9.33(\mathrm{~s}, 1 \mathrm{H}) ;{ }^{13} \mathrm{C} \mathrm{NMR}\left(\mathrm{CDCl}_{3}, 75 \mathrm{MHz}\right) \delta 101.09,126.23,126.40,127.27$, $128.53,128.59,128.79,128.99,129.10,129.60,129.98,132.44,133.31,145.49,149.10$, 151.56, 156.08; IR $\left(\mathrm{CHCl}_{3}\right)$ 3063, 3045, 1629, 1598, $1440 \mathrm{~cm}^{-1}$; HRMS m/z 381.0019 (calcd for $\mathrm{C}_{19} \mathrm{H}_{12} \mathrm{IN}, 381.0015$ ).

4-(2-Aminophenyl)-3-iodoquinoline (23). The reaction mixture was chromatographed using 3:1 hexane/EtOAc to afford $57 \mathrm{mg}$ (55\%) of quinoline 23 as a light yellow solid: mp 145-146 ${ }^{\circ} \mathrm{C} ;{ }^{1} \mathrm{H}$ NMR $\left(\mathrm{CDCl}_{3}, 400 \mathrm{MHz}\right) \delta 3.44$ (s, 2H), 6.88 (d, $J$ $=8.0 \mathrm{~Hz}, 1 \mathrm{H}), 6.94-6.98(\mathrm{~m}, 2 \mathrm{H}), 7.26-7.36(\mathrm{~m}, 1 \mathrm{H}), 7.45-7.47(\mathrm{~m}, 1 \mathrm{H}), 7.51-7.54(\mathrm{~m}$, 1H), 7.71-7.75 (m, 1H), $8.11(\mathrm{~d}, J=8.4 \mathrm{~Hz}, 1 \mathrm{H}), 9.24(\mathrm{~s}, 1 \mathrm{H}) ;{ }^{13} \mathrm{C} \mathrm{NMR}\left(\mathrm{CDCl}_{3}, 100\right.$ MHz) $\delta 97.64,116.05,118.79,125.67,126.48,127.86,128.83,129.69,129.81,130.12$, 130.14, 143.07, 147.38, 150.16, 156.94; IR $\left(\mathrm{CHCl}_{3}\right) 3100,2923,2851,1736,1451 \mathrm{~cm}^{-1}$; HRMS $m / z 345.9974$ (calcd for $\mathrm{C}_{15} \mathrm{H}_{11} \mathrm{IN}_{2}, 345.9967$ ). 


\section{Synthesis of 3-iodo-4-phenylquinoline (2) from $N$-phenyl- $N$-(3-phenyl-2-}

propyn-1-yl)methanesulfonamide (25) and 3-iodo-1-methanesulfonyl-4-phenyl-1,2dihydroquinoline (26). To a solution of $N$-phenylmethanesulfonamide ${ }^{5}(0.513 \mathrm{~g}, 3.0$ $\mathrm{mmol}), \mathrm{PPh}_{3}(1.18 \mathrm{~g}, 4.5 \mathrm{mmol})$ and 3-phenyl-2-propyn-1-ol (0.594 g, $\left.4.5 \mathrm{mmol}\right)$ in anhydrous THF $(30 \mathrm{~mL})$ at $0{ }^{\circ} \mathrm{C}$ was added DEAD $(0.784 \mathrm{~g}, 4.5 \mathrm{mmol})$. The resulting solution was stirred at $0{ }^{\circ} \mathrm{C}$ for $1 \mathrm{~h}$ and an additional $3 \mathrm{~h}$ at room temperature. The mixture was washed with brine $(30 \mathrm{~mL})$ and the organic layer was dried over $\mathrm{Na}_{2} \mathrm{SO}_{4}$, filtered, and the solvent removed under reduced pressure. The residue was purified by chromatography on a silica gel column using 3:1 hexane/ethyl acetate to obtain $0.534 \mathrm{~g}$ ( $63 \%)$ of $N$-phenyl- $N$-(3-phenyl-2-propyn-1-yl)methanesulfonamide (25) as a white solid: mp 76-77 ${ }^{\circ} \mathrm{C} ;{ }^{1} \mathrm{H}$ NMR $\left(\mathrm{CDCl}_{3}\right) \delta 3.08$ (s, 3H), 4.67 (s, 2H), 7.34-7.46 (m, 8H), 7.62-7.66 (m, 2H); ${ }^{13} \mathrm{C}$ NMR $\left(\mathrm{CDCl}_{3}\right) \delta 39.2,42.3,84.4,86.3,122.3,127.7,128.4,128.7,129.1$, 129.7, 131.9, 140.5. To a solution of $\quad N$-phenyl- $N$-(3-phenyl-2-propyn-1yl)methanesulfonamide $(71.2 \mathrm{mg}, 0.25 \mathrm{mmol})$ in $\mathrm{CH}_{2} \mathrm{Cl}_{2}(3.0 \mathrm{~mL})$ at $-78{ }^{\circ} \mathrm{C}$ was added ICl (48.7 mg, $0.3 \mathrm{mmol})$ in $\mathrm{CH}_{2} \mathrm{Cl}_{2}(0.5 \mathrm{~mL})$ and the resulting solution was stirred at this temperature for $1 \mathrm{~h}$. The reaction mixture was washed with satd aq $\mathrm{Na}_{2} \mathrm{~S}_{2} \mathrm{O}_{3}(20 \mathrm{~mL})$ and the organic layer dried over $\mathrm{Na}_{2} \mathrm{SO}_{4}$, filtered and the solvent removed under reduced pressure. The residue was purified by chromatography on a silica gel column using 5:1 hexane/ethyl acetate to obtain $82.2 \mathrm{mg}(80 \%)$ of 3-iodo-1-methanesulfonyl-4-phenyl1,2-dihydroquinoline (26) as a white solid: mp $173-175{ }^{\circ} \mathrm{C} ;{ }^{1} \mathrm{H}$ NMR $\left(\mathrm{CDCl}_{3}\right) \delta 2.89$ (s, $3 \mathrm{H}), 4.82(\mathrm{~s}, 2 \mathrm{H}), 6.80(\mathrm{dd}, J=7.8,1.2 \mathrm{~Hz}, 1 \mathrm{H}), 7.11-7.16(\mathrm{~m}, 3 \mathrm{H}), 7.30-7.33(\mathrm{~m}, 1 \mathrm{H})$, 7.44-7.48 (m, 3H), $7.62(\mathrm{dd}, J=8.1,0.9 \mathrm{~Hz}, 1 \mathrm{H}) ;{ }^{13} \mathrm{C} \mathrm{NMR}\left(\mathrm{CDCl}_{3}\right) \delta 38.6,56.7,92.0$, $126.9,127.2,127.2128 .6,128.8,129.0,129.2,130.7,134.5,140.3,143.9$. A solution of 
3-iodo-1-methanesulfonyl-4-phenyl-1,2-dihydroquinoline (0.103 g, $0.25 \mathrm{mmol})$ and $\mathrm{NaOH}(0.10 \mathrm{~g}, 2.5 \mathrm{mmol})$ in $\mathrm{EtOH}(10 \mathrm{~mL})$ was stirred at $50{ }^{\circ} \mathrm{C}$ under $\mathrm{O}_{2}(1 \mathrm{~atm})$ for 12 h. The reaction mixture was diluted with diethyl ether $(50 \mathrm{~mL})$ and washed with brine $(50 \mathrm{~mL})$. The organic layer was dried $\left(\mathrm{Na}_{2} \mathrm{SO}_{4}\right)$, filtered, and the solvent removed under reduced pressure. The residue was purified by column chromatography on a silica gel column using 5:1 hexanes/ethyl acetate to afford $76.1 \mathrm{mg}(92 \%)$ of the desired compound 2.

4-(4-Fluorophenyl)-3-[(E)-2-phenylethenyl]quinoline (27). To a solution of 4(4-fluorophenyl)-3-iodoquinoline $(0.15 \mathrm{mmol})$ and $\beta$-styreneboronic acid $(0.23 \mathrm{mmol}, 1.5$ equiv) in $10 \mathrm{~mL}$ of $\mathrm{DMF} / \mathrm{H}_{2} \mathrm{O}(\mathrm{V} / \mathrm{V}=4 / 1)$ were added $\mathrm{PdCl}_{2}\left(\mathrm{PPh}_{3}\right)_{2}(2.0 \mathrm{mg}, 5 \mathrm{~mol} \%)$ and $\mathrm{K}_{2} \mathrm{CO}_{3}\left(0.30 \mathrm{mmol}, 2.0\right.$ equiv). The resulting mixture was heated under an $\mathrm{N}_{2}$ atmosphere at $100{ }^{\circ} \mathrm{C}$ for $2 \mathrm{~h}$. The mixture was cooled to room temperature and diluted with $25 \mathrm{ml}$ of ether, washed with $25 \mathrm{~mL}$ of satd aq $\mathrm{NaCl}$, dried over $\mathrm{MgSO}_{4}$ and filtered. The solvent was evaporated under reduced pressure. The reaction mixture was chromatographed using 3:1 hexane/EtOAc to afford $35.4 \mathrm{mg}(73 \%)$ of the product as a light yellow solid: mp $178-180{ }^{\circ} \mathrm{C} ;{ }^{1} \mathrm{H}$ NMR $\left(\mathrm{CDCl}_{3}, 300 \mathrm{MHz}\right) \delta 6.91(\mathrm{~d}, J=16.5 \mathrm{~Hz}$, $1 \mathrm{H}), 7.23-7.38(\mathrm{~m}, 11 \mathrm{H}), 7.45-7.52(\mathrm{~m}, 2 \mathrm{H}), 7.65-7.70(\mathrm{~m}, 1 \mathrm{H}), 8.15(\mathrm{~d}, J=8.4 \mathrm{~Hz}, 1 \mathrm{H})$, 9.33 (s, $1 \mathrm{H}) ;{ }^{13} \mathrm{C} \mathrm{NMR}\left(\mathrm{CDCl}_{3}, 75 \mathrm{MHz}\right) 115.68,115.89,124.38,126.26,126.72,127.10$, $127.45,128.15,128.24,128.80,129.04,129.61,131.44,131.72,131.76,131.94,132.02$, 136.92, 144.02, 147.31, 148.47, 161.49, 163.96; IR $\left(\mathrm{CHCl}_{3}\right) 2922,1605,1514,1499 \mathrm{~cm}^{-}$ ${ }^{1}$; HRMS m/z 325.1272 (calcd for $\mathrm{C}_{23} \mathrm{H}_{16} \mathrm{FN}, 325.1267$ ).

7H-Indolo[2,3-c]quinoline (28). To a solution of 4-(2-aminophenyl)-3iodoquinoline $(0.15 \mathrm{mmol})$ in $4 \mathrm{~mL}$ of toluene/DMF $(\mathrm{V} / \mathrm{V}=3 / 1)$ in a vial was added 
$\mathrm{PdCl}_{2}$ (dppf) (4.9 mg, $\left.5 \mathrm{~mol} \%\right)$, dppf (4.2 $\left.\mathrm{mg}, 5 \mathrm{~mol} \%\right)$, and $t$-BuOK (0.30 mmol, 2.0 equiv). Then the vial was flushed with $\mathrm{N}_{2}$ gas and closed. The reaction mixture was heated to $100{ }^{\circ} \mathrm{C}$ for $36 \mathrm{~h}$, cooled to room temperature, diluted with $25 \mathrm{ml}$ of ether, washed with $25 \mathrm{~mL}$ of satd aq $\mathrm{NaCl}$, dried over $\mathrm{MgSO}_{4}$ and filtered. The solvent was evaporated under reduced pressure. The reaction mixture was chromatographed using 1:1 hexane/EtOAc to afford $21 \mathrm{mg}(65 \%)$ of the product as a light yellow solid: $\mathrm{mp} 247$ $248{ }^{\circ} \mathrm{C}$ (lit. mp $\left.249{ }^{\circ} \mathrm{C}\right) ;{ }^{6}{ }^{1} \mathrm{H}$ NMR $(d$-acetone, $400 \mathrm{MHz}) \delta 7.45-7.48(\mathrm{~m}, 1 \mathrm{H}), 7.60-7.63$ (m, 1H), 7.68-7.72 (m, 2H), 7.75-7.79 (m, 1H), $8.33(\mathrm{~d}, J=8.0 \mathrm{~Hz}, 1 \mathrm{H}), 8.60(\mathrm{~d}, J=8.0$ $\mathrm{Hz}, 1 \mathrm{H}), 8.74(\mathrm{~d}, J=8.0 \mathrm{~Hz}, 1 \mathrm{H}), 9.15(\mathrm{~s}, 1 \mathrm{H}), 9.29(\mathrm{~s}, 1 \mathrm{H}) ;{ }^{13} \mathrm{C}$ NMR $(d$-acetone, 100 MHz) 112.55, 112.60, 120.51, 123.03, 123.32, 124.80, 125.20, 126.72, 126.94, 130.45, 138.63, 138.68, 143.10 (two sp ${ }^{2}$ carbons missing due to overlap); IR $\left(\mathrm{CHCl}_{3}\right) 3135,3075$, 2849, 1922, 1736, $1564 \mathrm{~cm}^{-1}$; HRMS m/z 218.0847 (calcd for $\mathrm{C}_{15} \mathrm{H}_{10} \mathrm{~N}_{2}, 218.0844$ ).

\section{References}

(1) (a) Yoshida, Y.; Tanabe, Y. Synthesis 1997, 533. (b) Barluenga, J.; Campos, P. J.;

Canal, G. Synthesis 1989, 33.

(2) Marshall, J. A.; Wolf, M. A. J. Org. Chem. 1996, 61, 3238.

(3) For the synthesis of propargylic mesylates from propargyl alcohol, see: Tanabe, Y.; Yamamoto, H.; Yoshida, Y.; Miyawaki, T.; Utsumi, N. Bull. Chem. Soc. Jpn. 1995, 68, 297.

(4) Andreev, V. P. J. Org. Chem. USSR (Engl. Transl.) 1979, 15, 414.

(5) Inoue, J.; Cui, Y.-S.; Sakai, O.; Nakamura, Y.; Kogiso, H.; Kador, P. F. Bioorg. Chem. 2000, 8, 2167. 
(6) Clemo, G. R.; Felton, D. G. I. J. Chem. Soc. 1951, 671. 

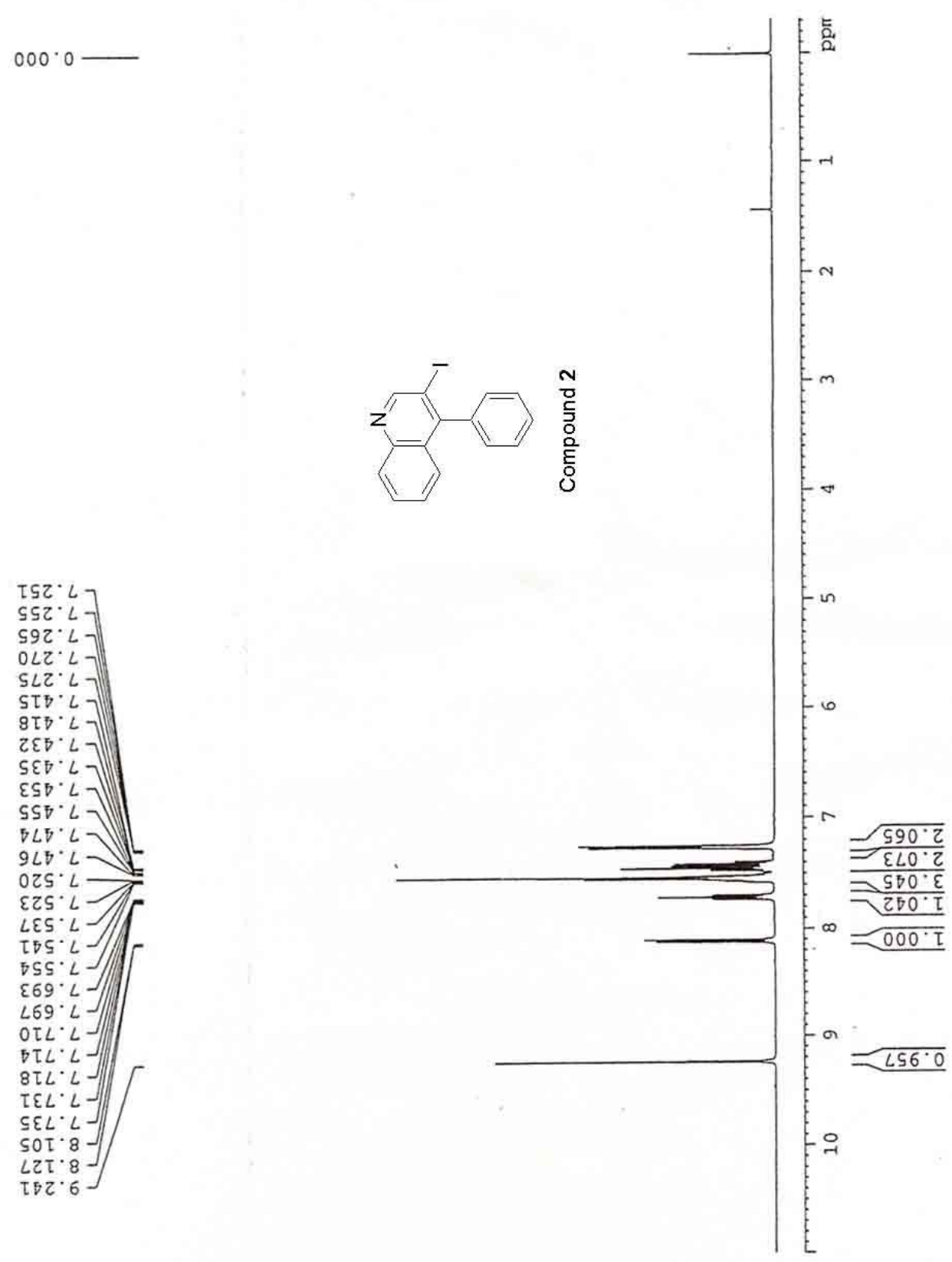


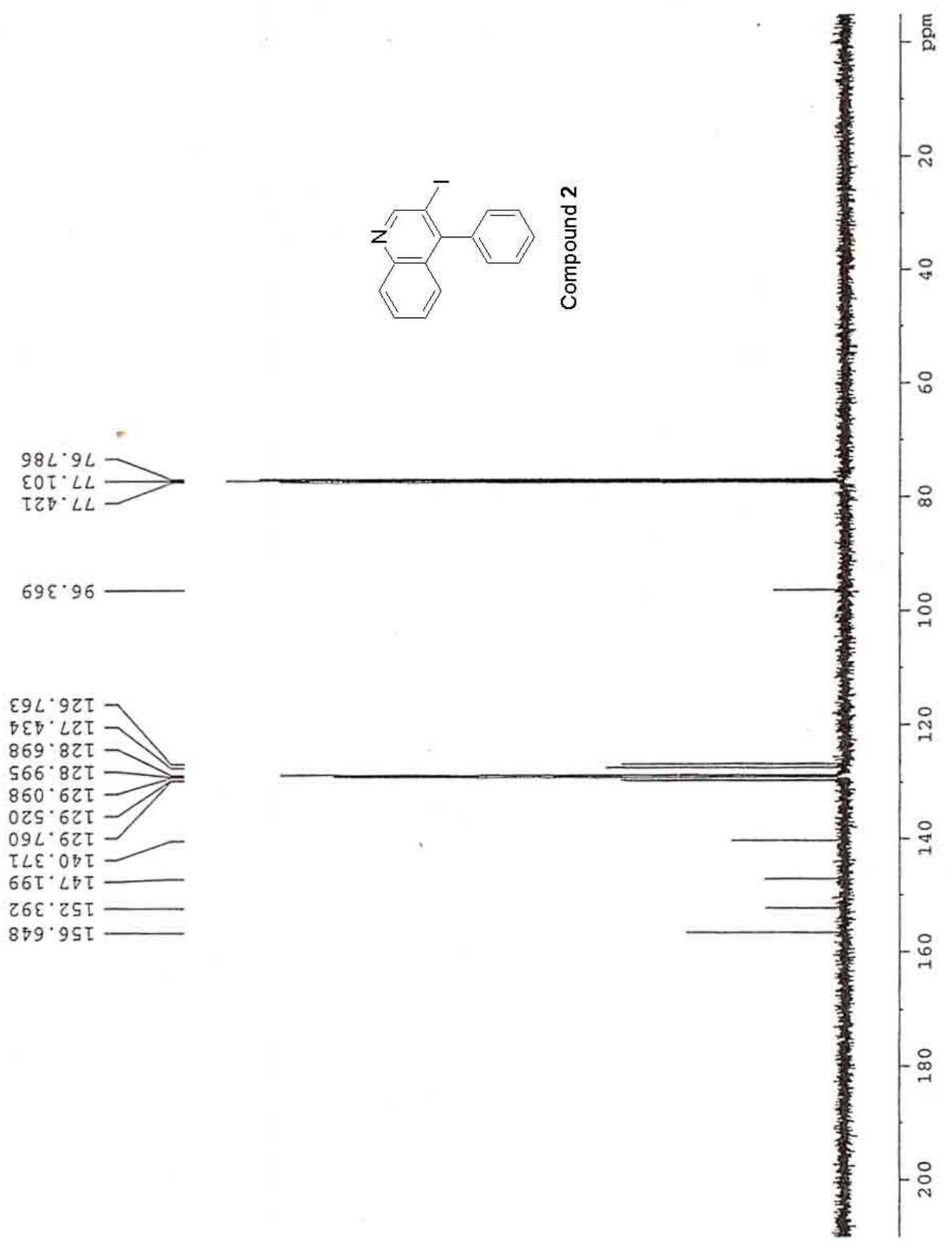




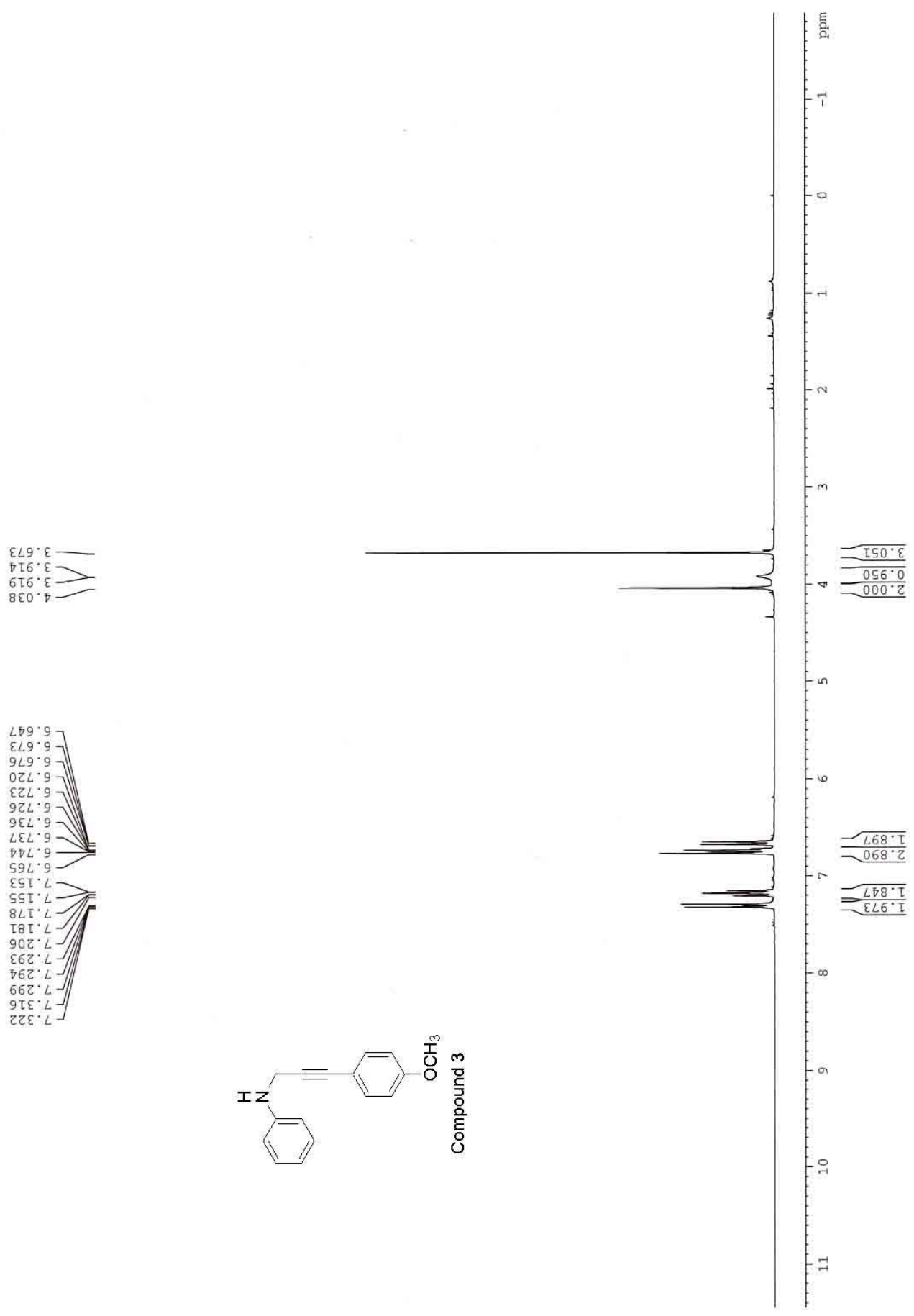



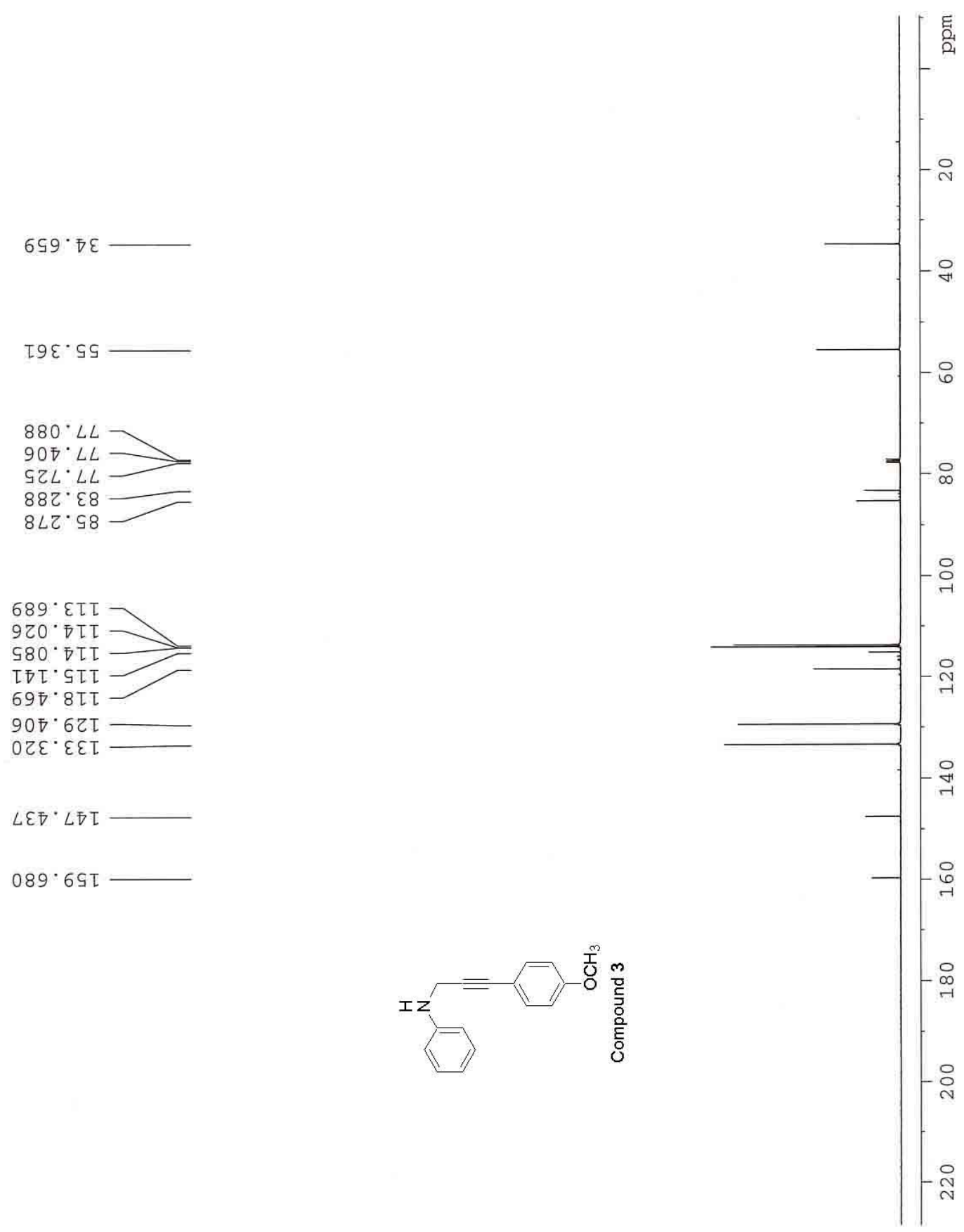


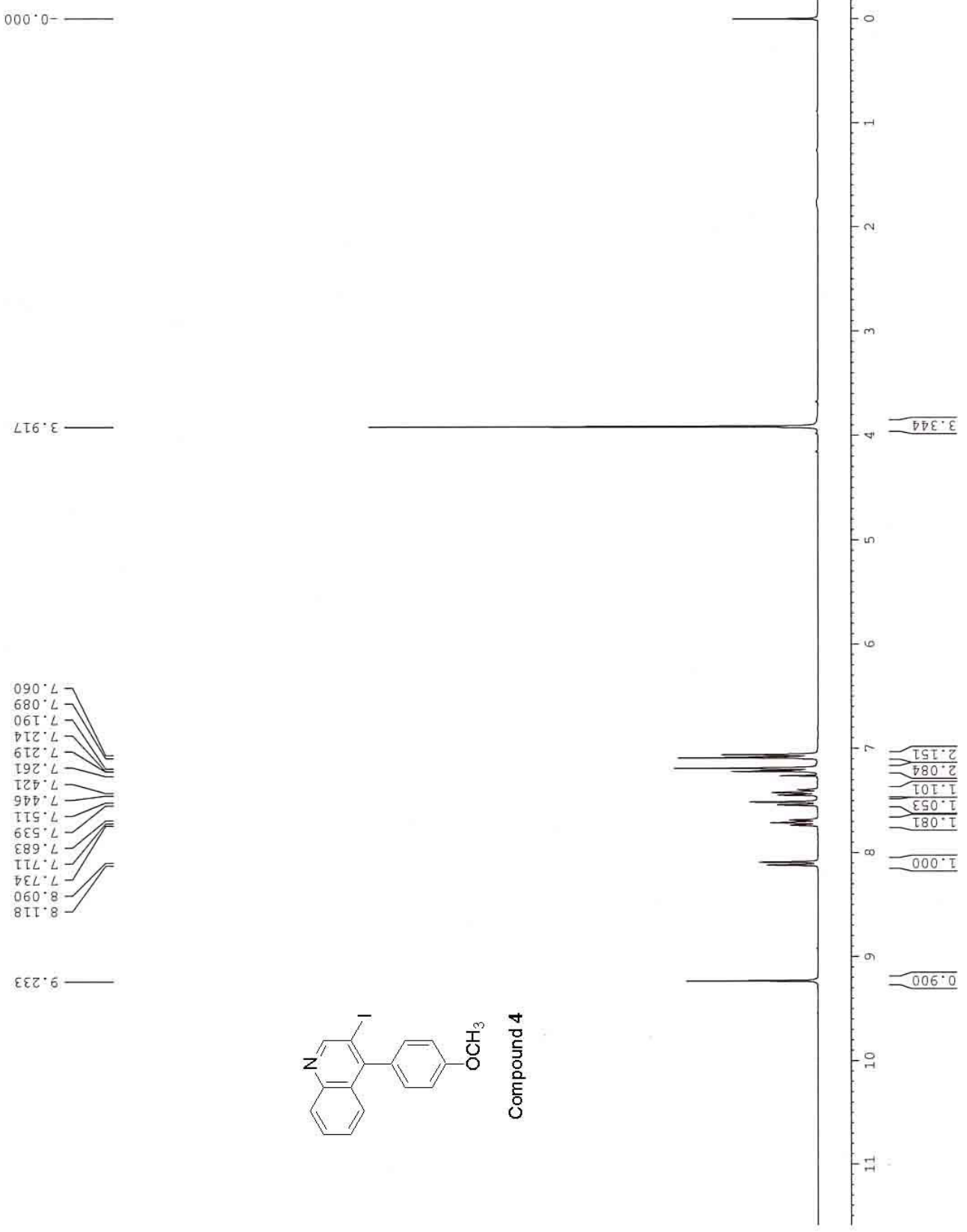



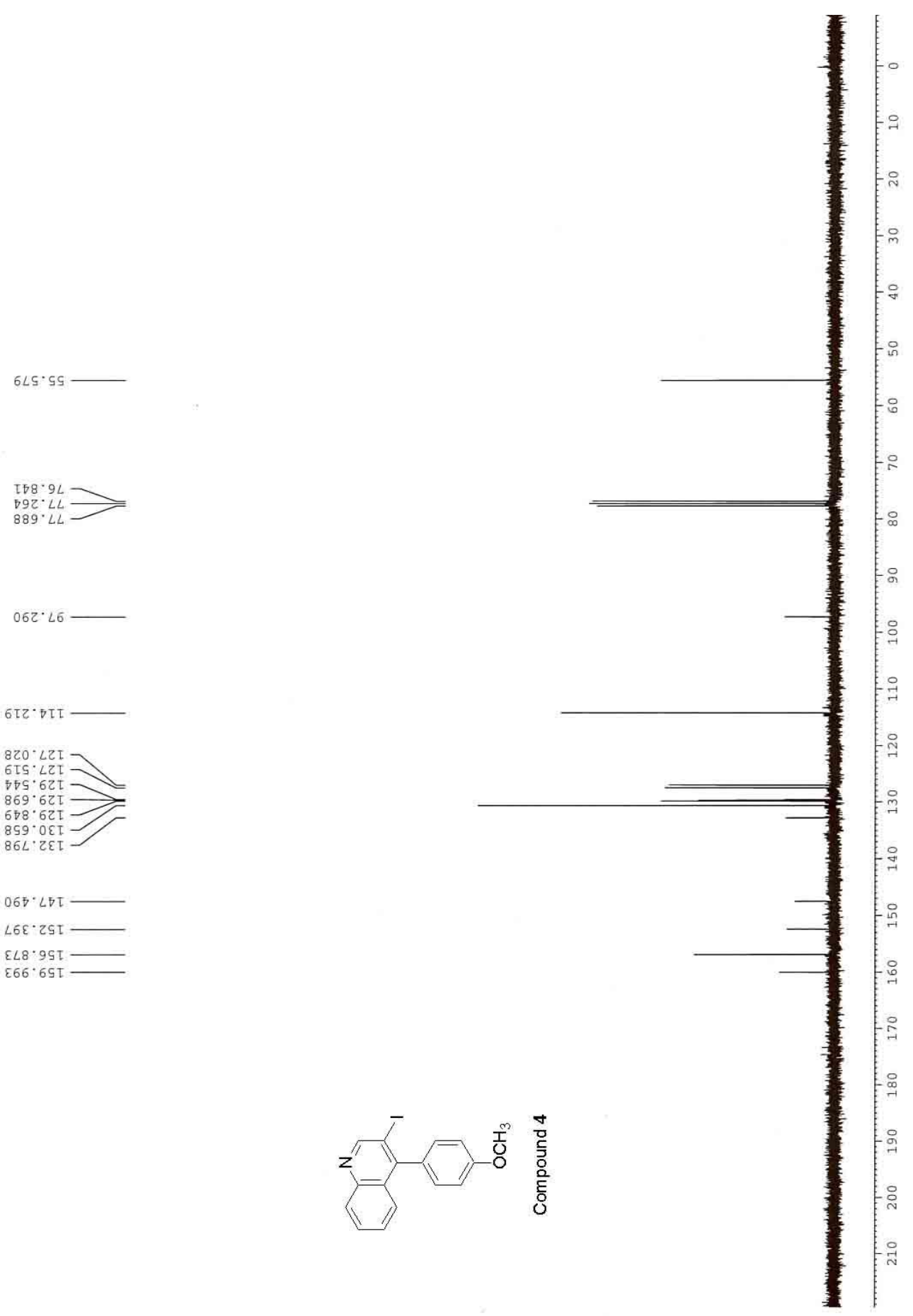

ำ 

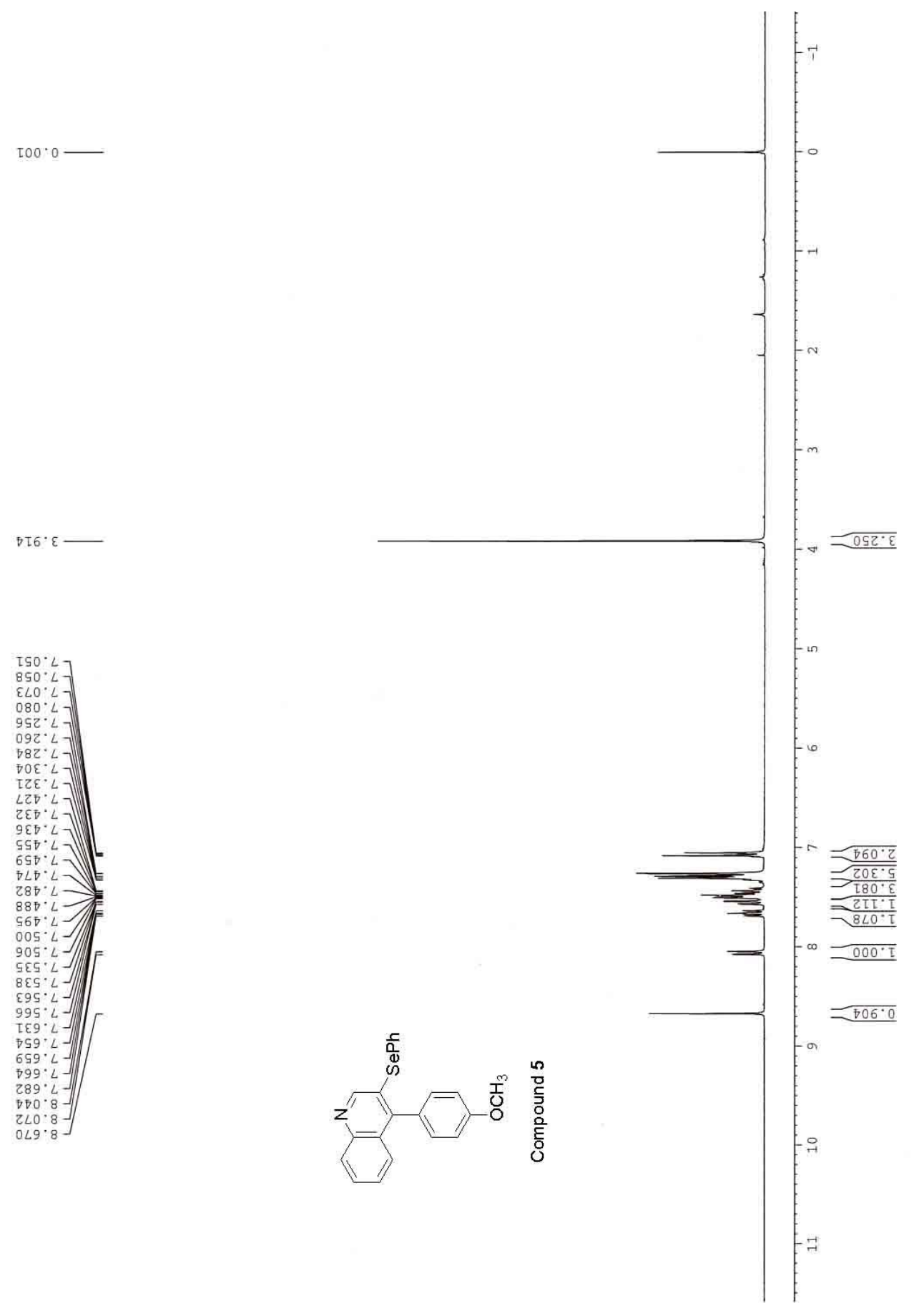

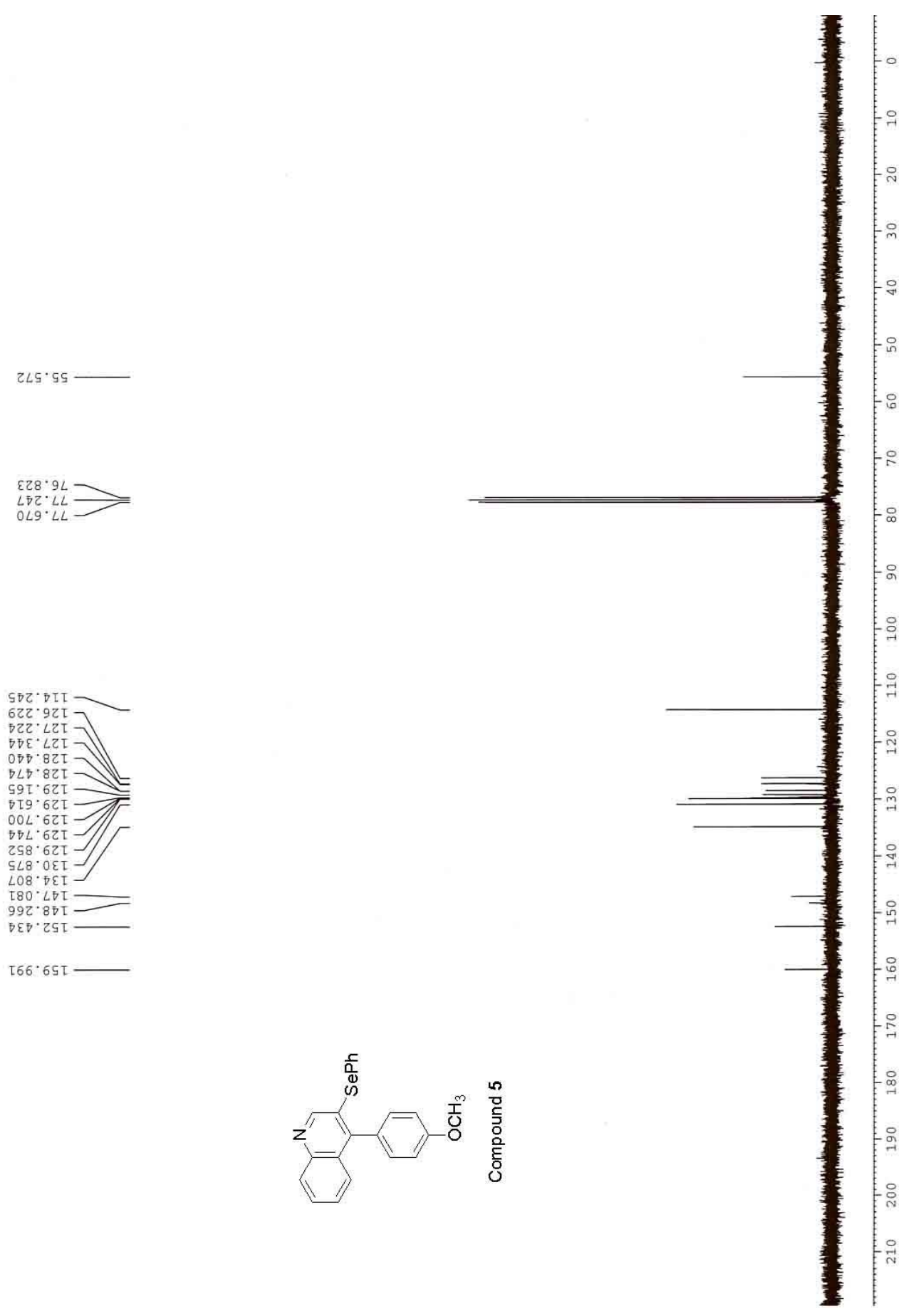

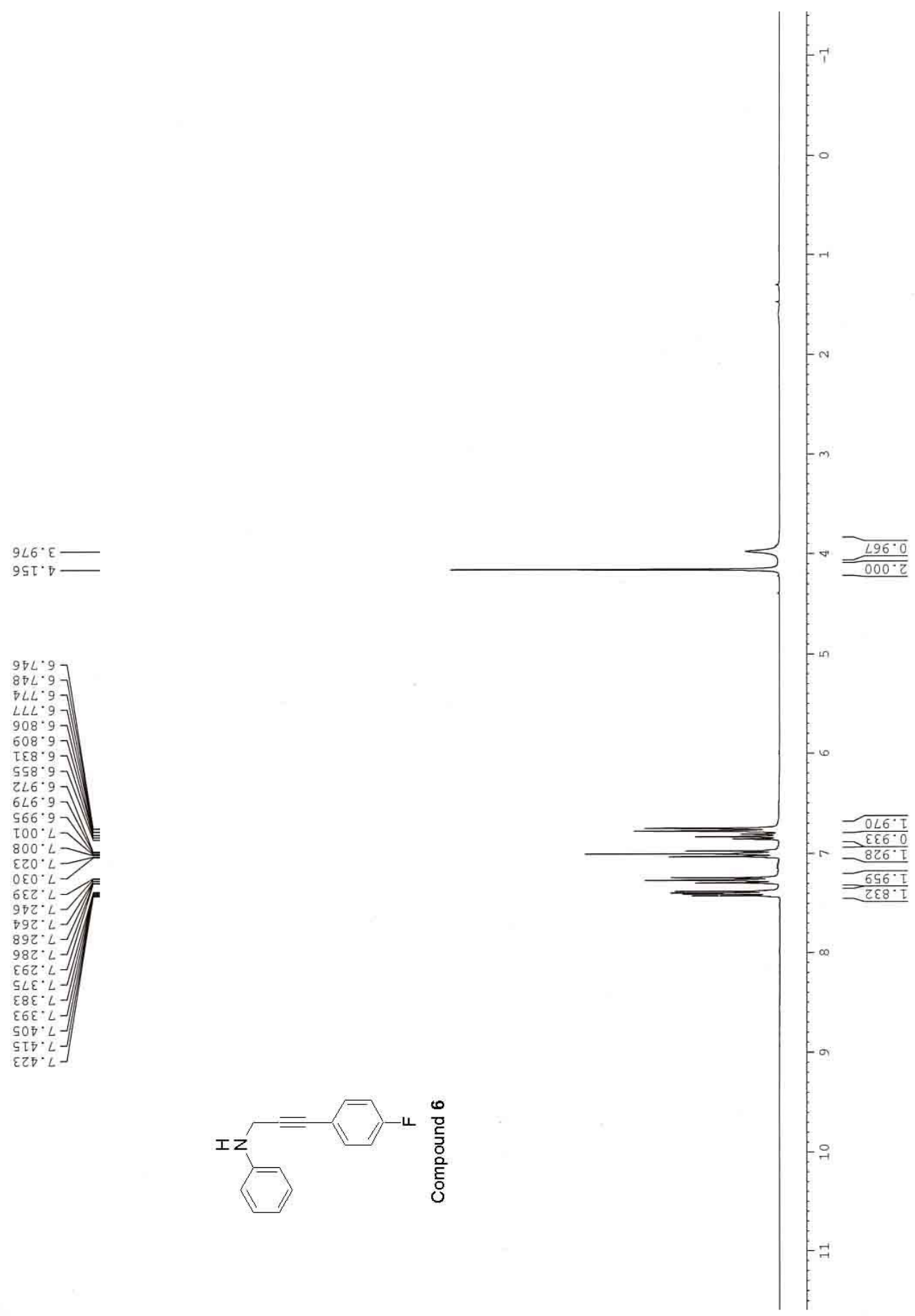
$8 Z L \cdot \nabla \varepsilon$

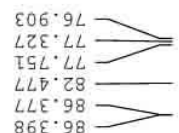

$8 Z 8 \cdot \varepsilon \tau \tau$ I万9. 9 II ปह6. SIT

$98 L^{\circ} 8 \mathrm{II} \longrightarrow$

$\varepsilon \varepsilon Z^{\circ}$. IT

$025 \cdot 62 t$

ป็ $6 \cdot \varepsilon \varepsilon \tau \longrightarrow$

$95 \varepsilon^{\circ} \angle D I-$

IS0. T9T

9รE. च9T

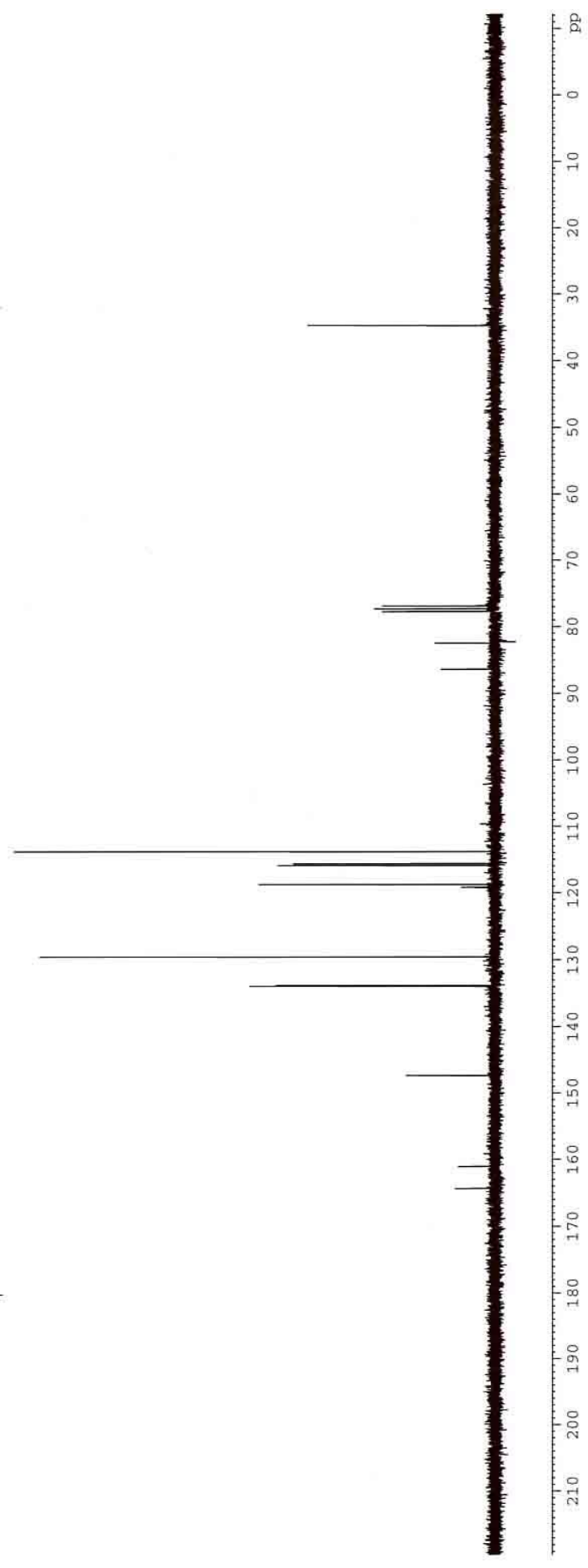

オ 

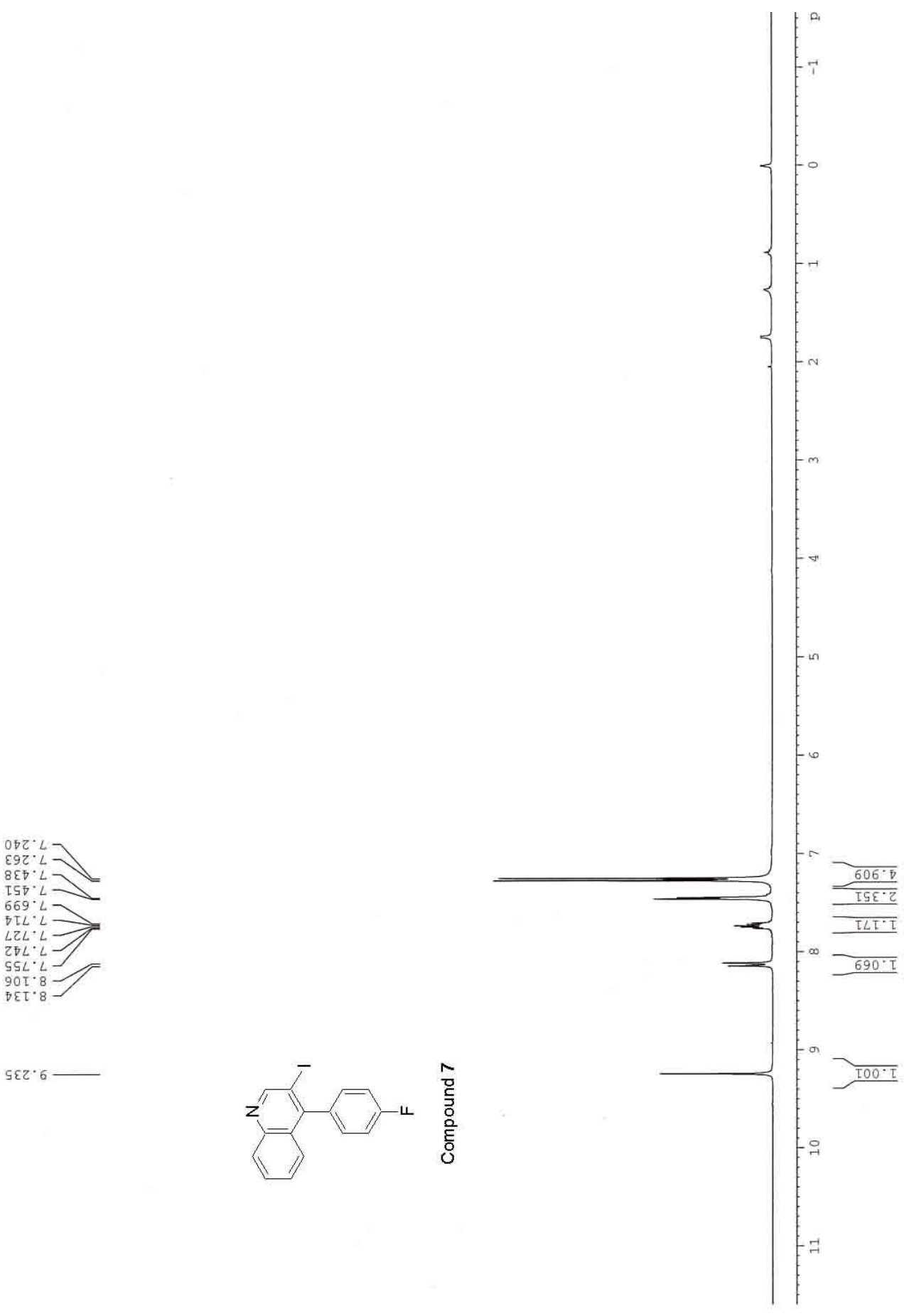

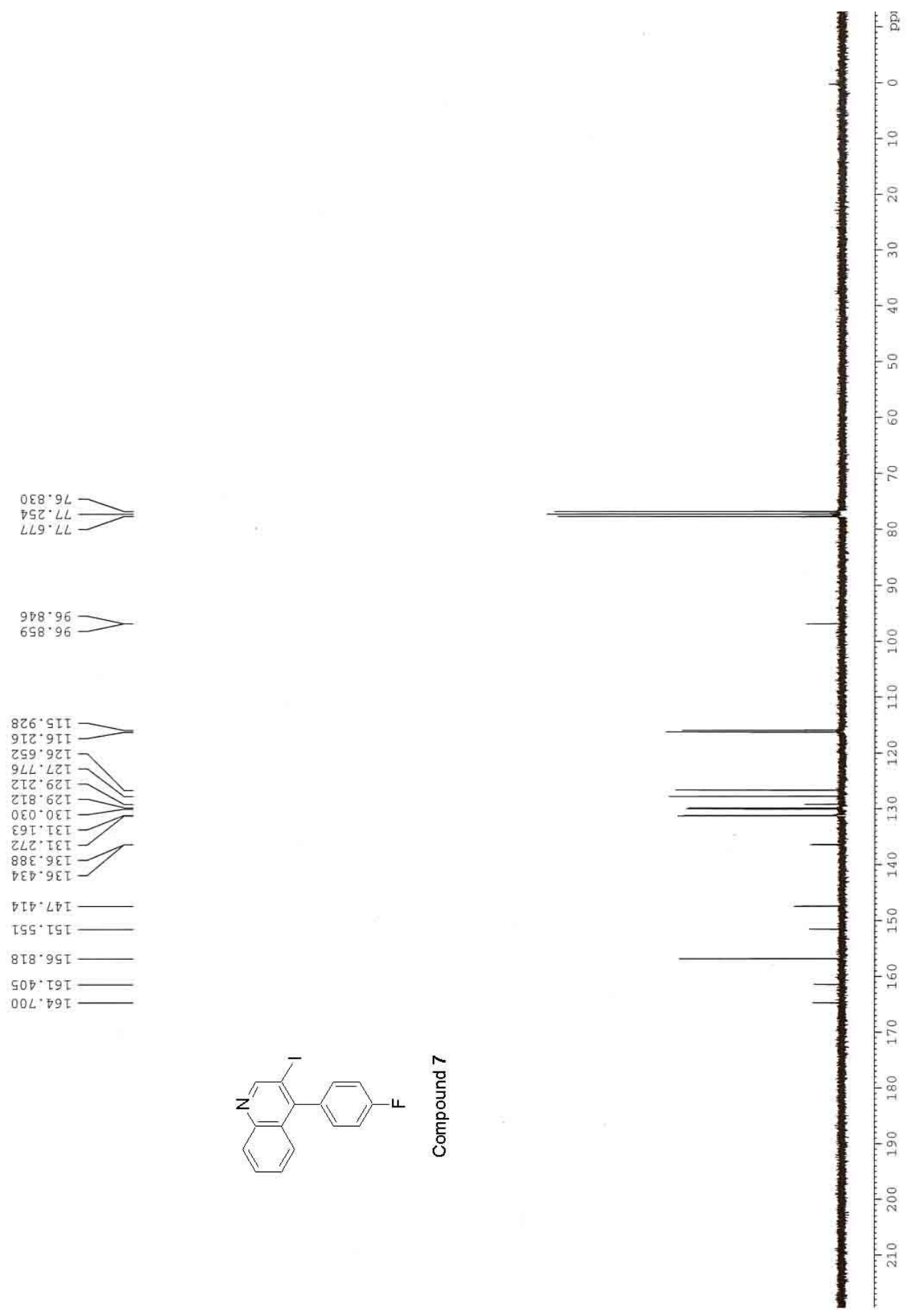

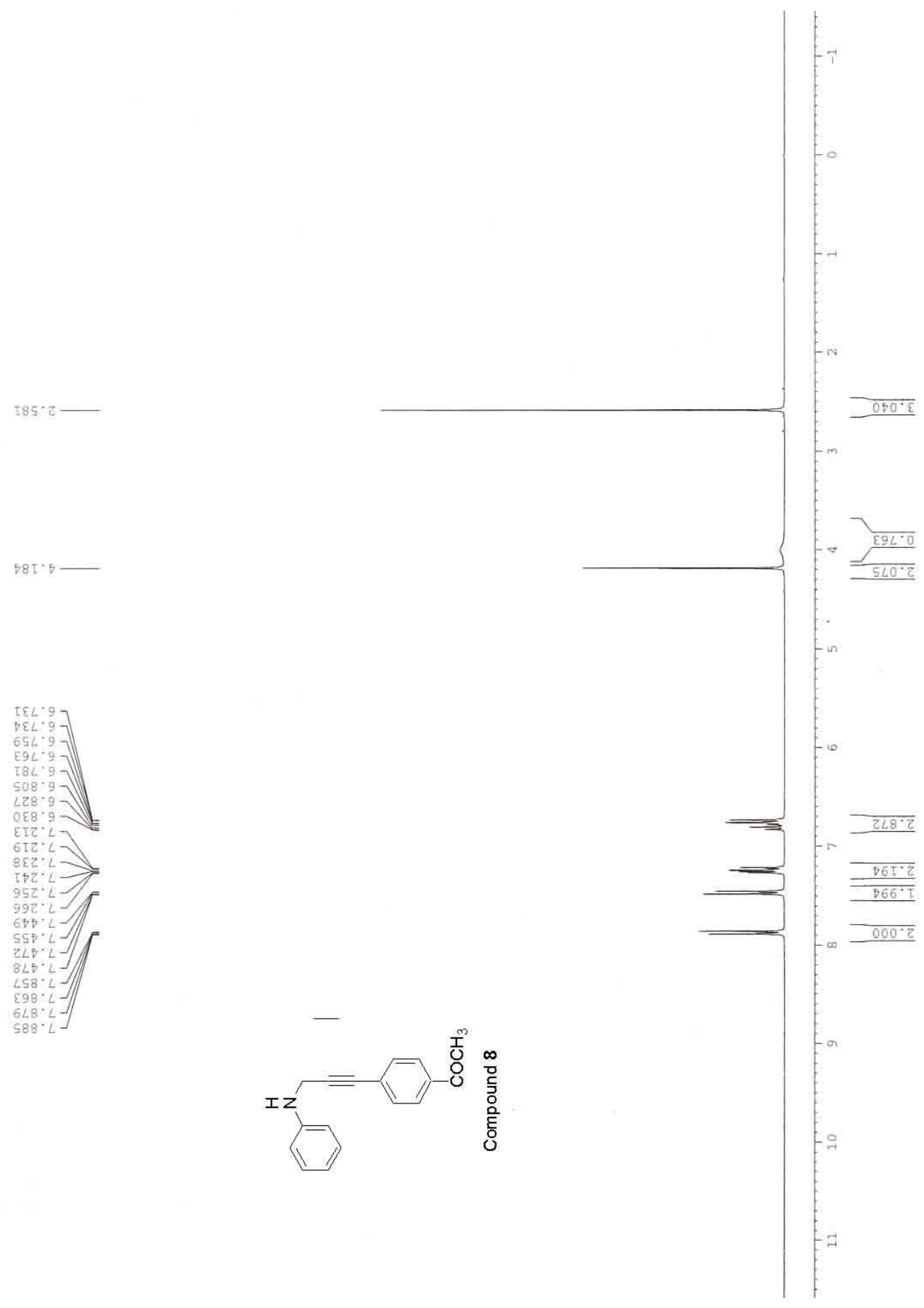

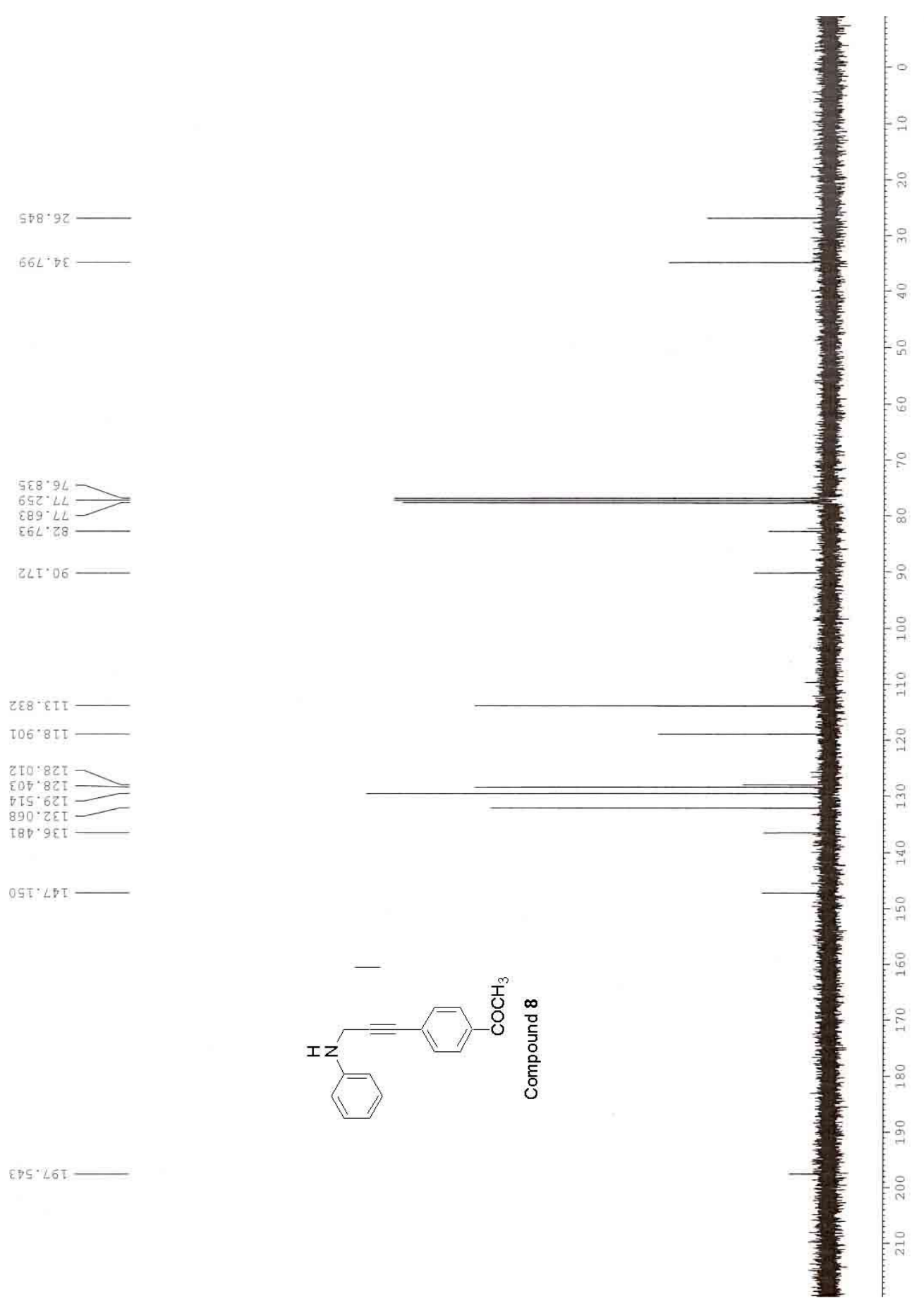


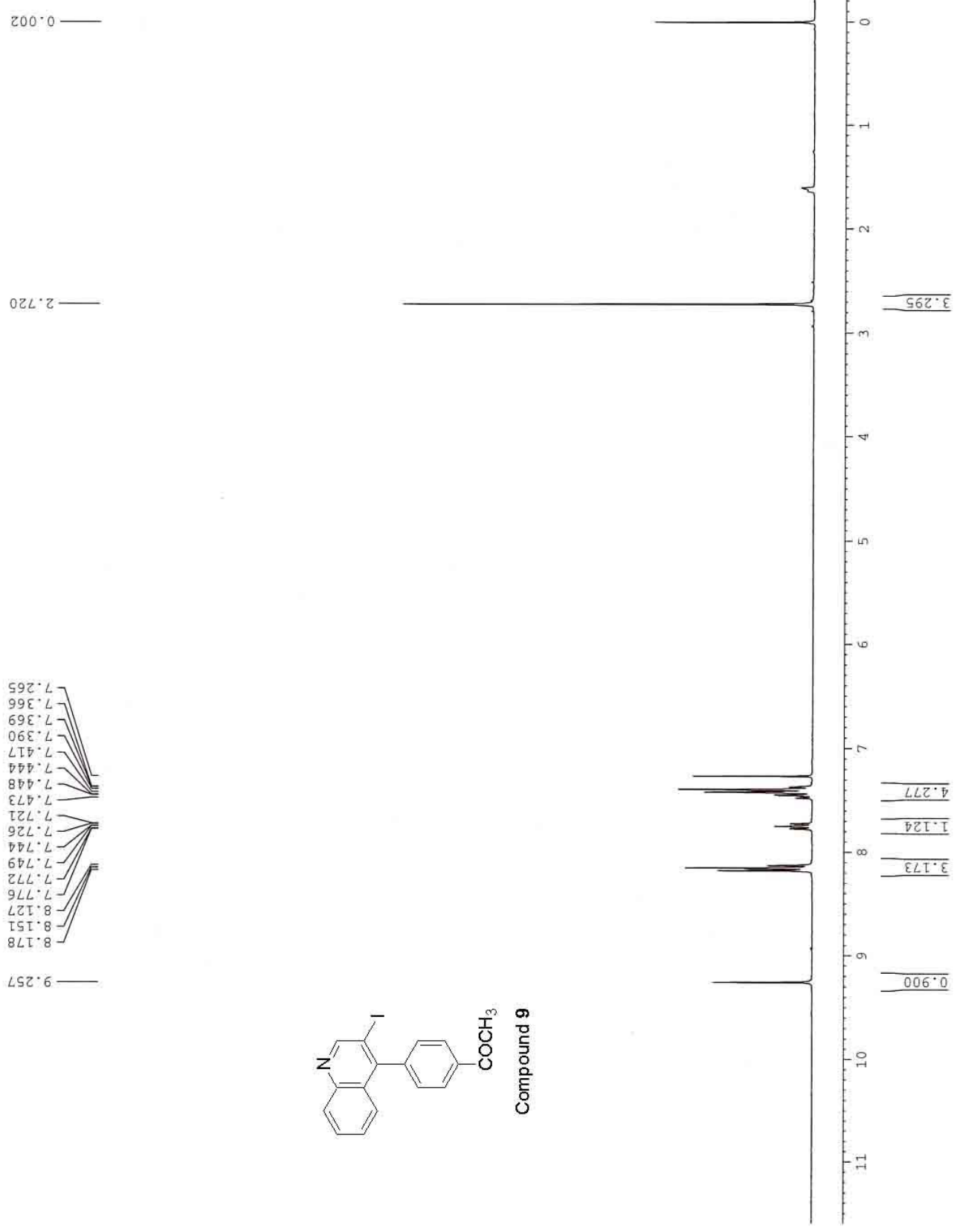




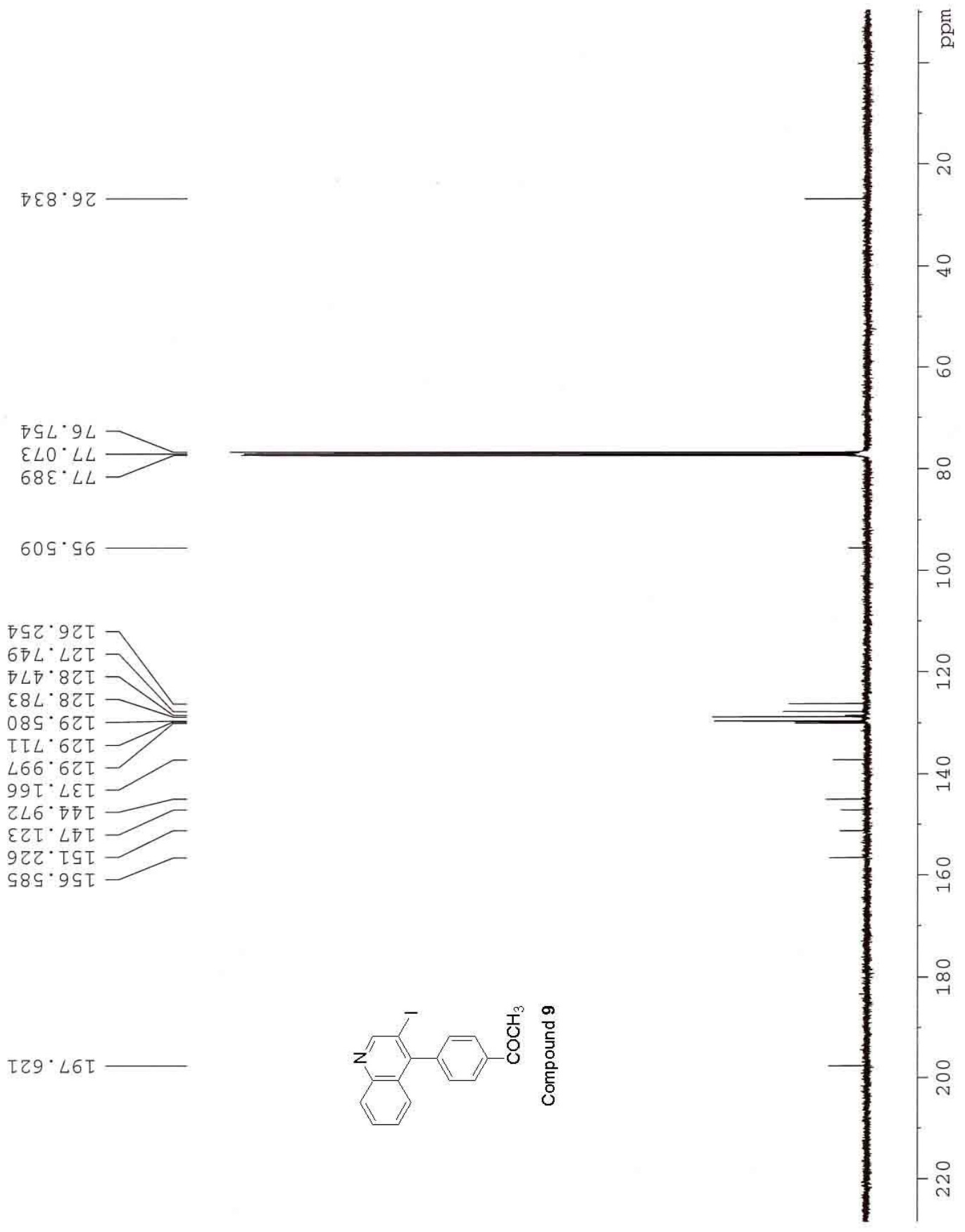



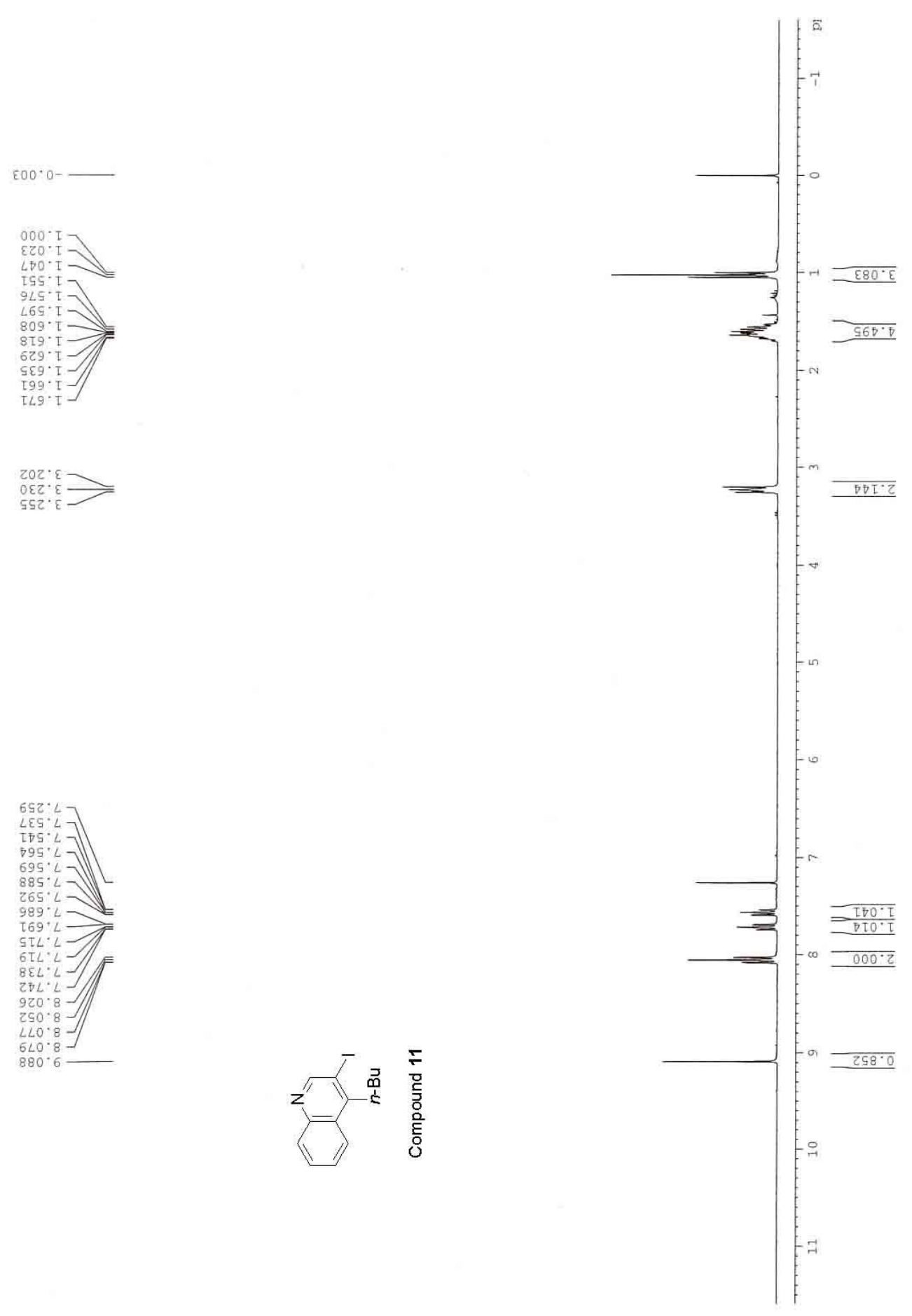


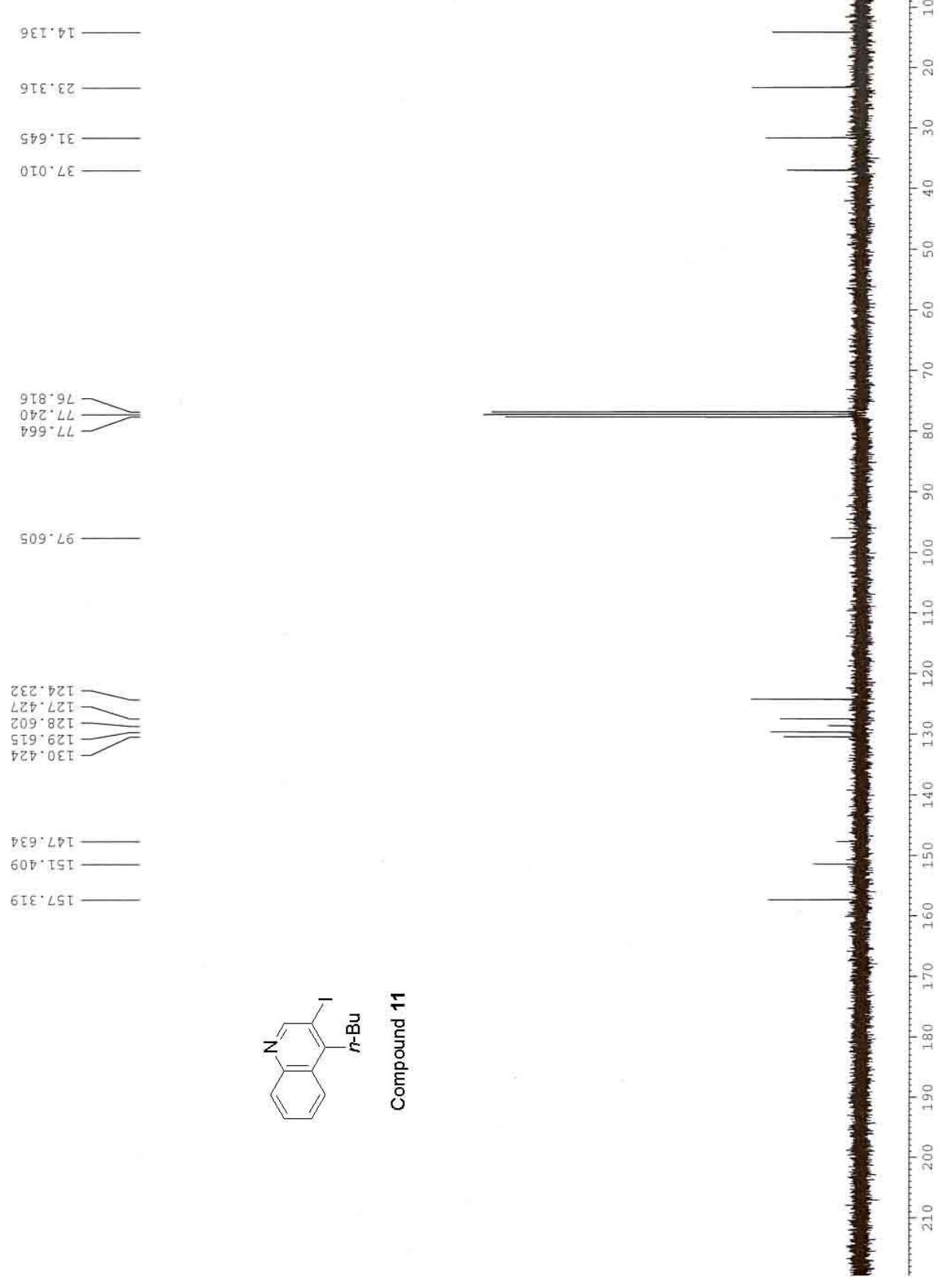




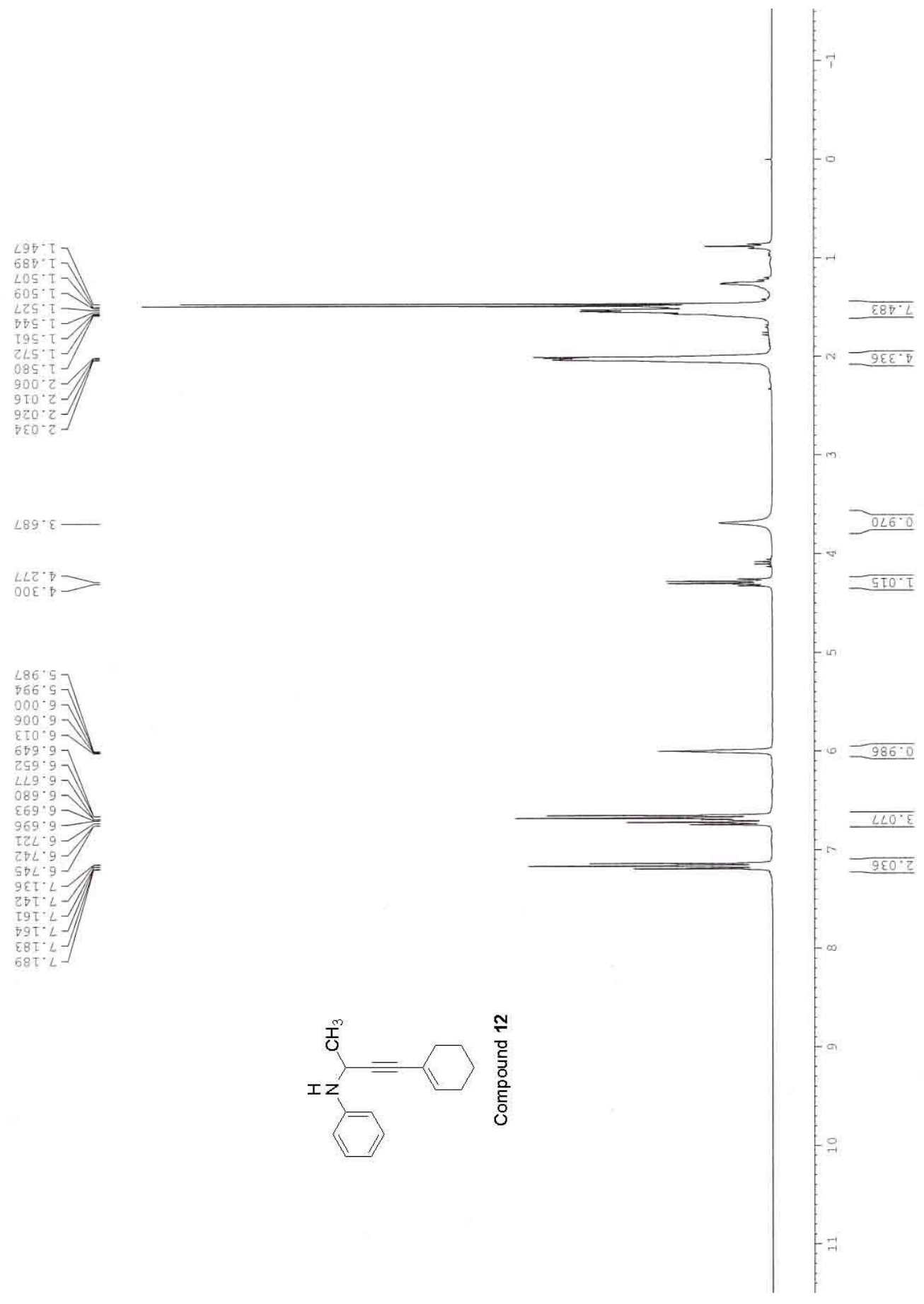




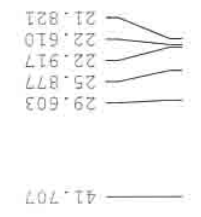

LOL'TH
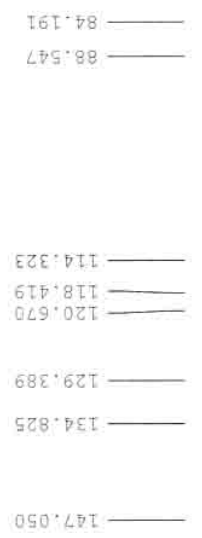

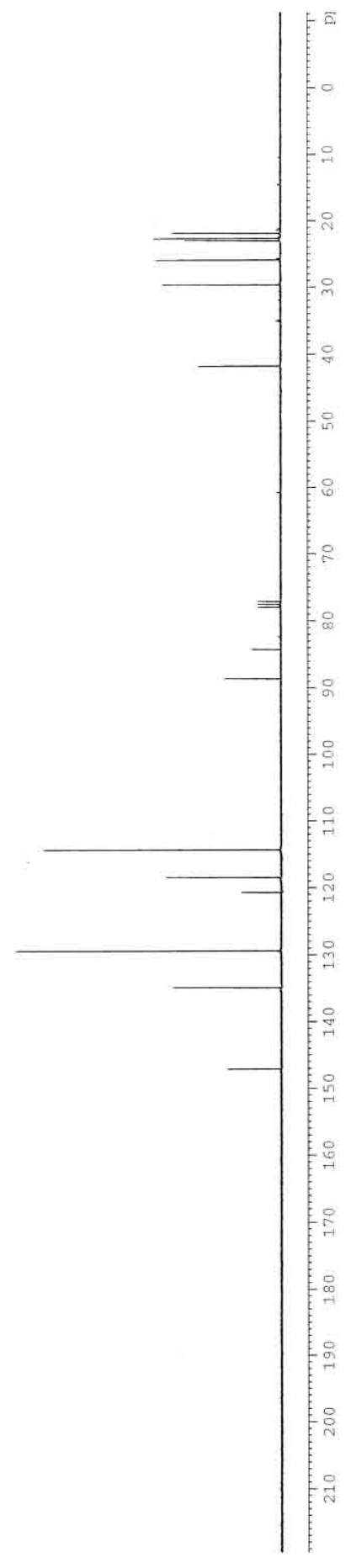

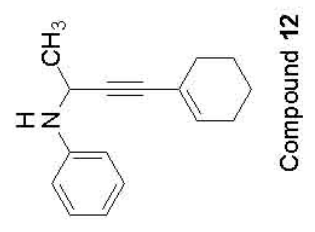



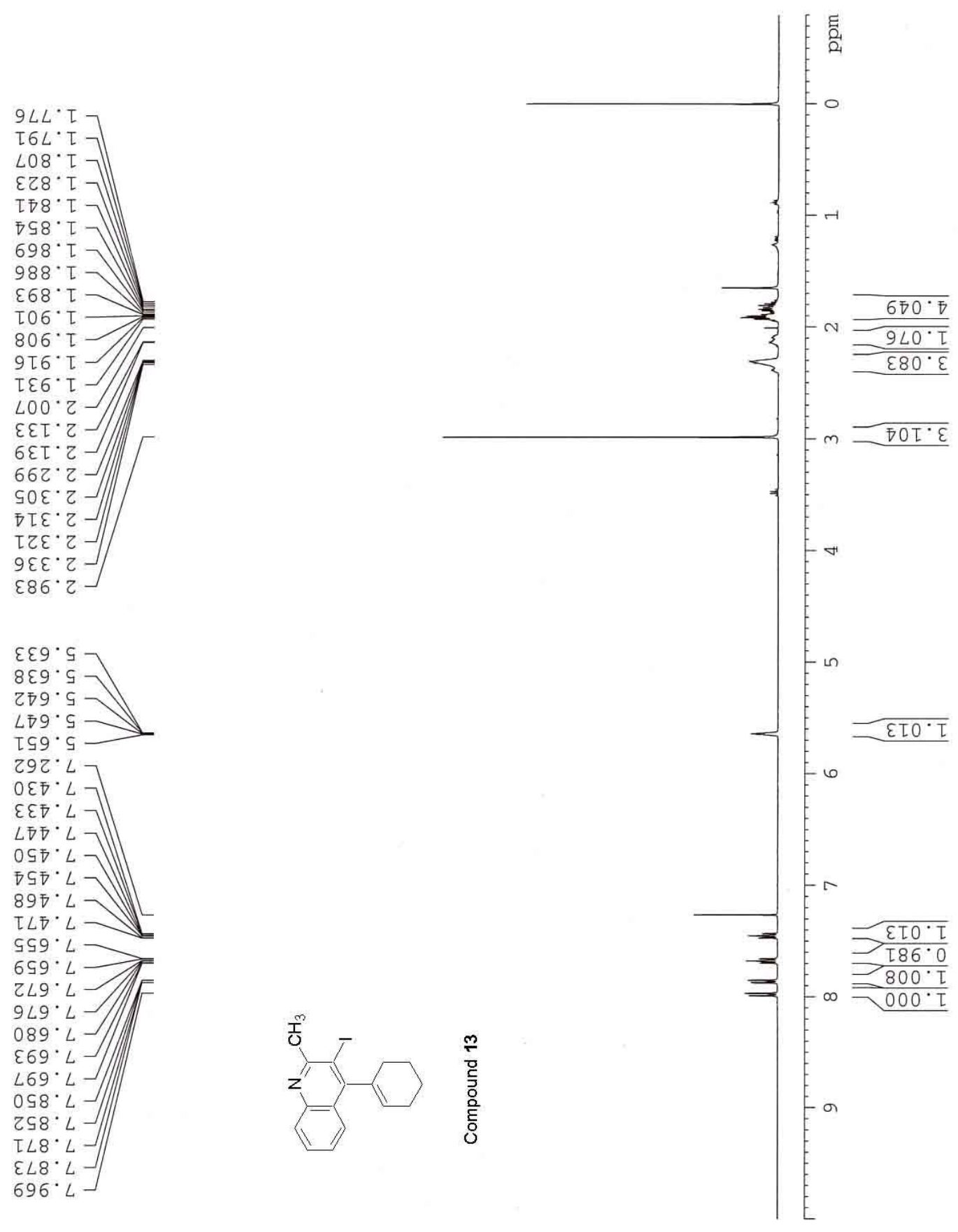
ZL6. IZ

$89 \mathrm{~L} \cdot \mathrm{ZZ} \longrightarrow$

67て.ร乙

$8 L L \cdot 8 Z$

$90 \nabla \cdot \tau \varepsilon=$

$\angle \bar{D} L \cdot 9 L$ $\$ 90^{\circ} \mathrm{LL}$

Z8.

$8 Z 币 \cdot 66$

8L6.ร乙โ

$\angle 9 Z^{\circ} 9 Z I$

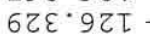

$659^{\circ} 8 \mathrm{ZI}$
$\varepsilon 08^{\circ} 8 \mathrm{ZT}$

ह08.8ZI

$9 \varepsilon \varepsilon \cdot 6 \varepsilon T$

ร6 $6^{\circ} 9 \mp \tau$

$9 L L$ SSI

IZ6.6ST

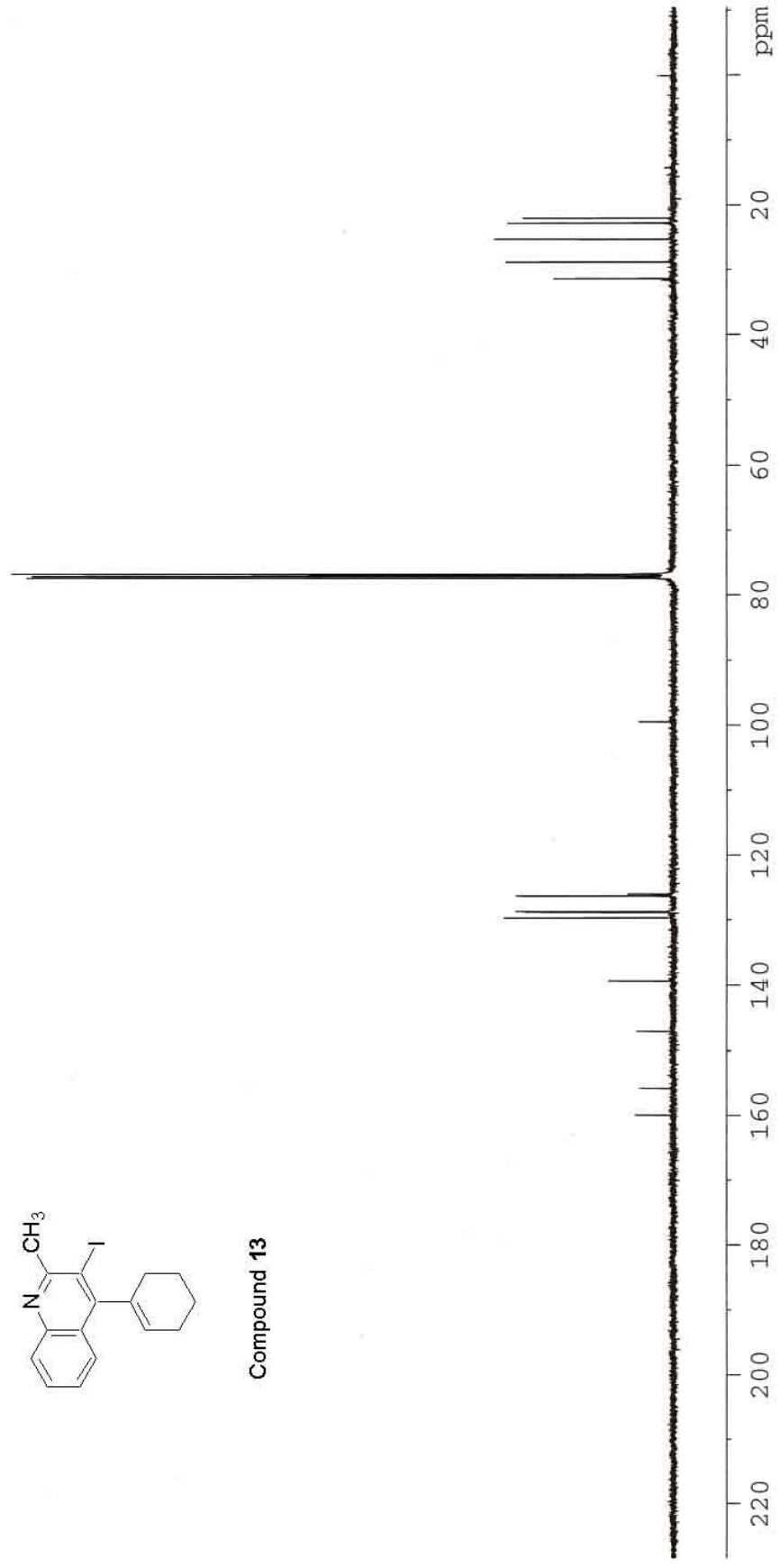


$800 \cdot 0$

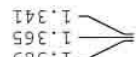

6BE. I

$26 \tau \cdot 0$
$262 \cdot 7$

$9 \tau \varepsilon \cdot \nabla$

$0 \nabla \varepsilon \cdot \nabla$
$\nabla 9 \varepsilon \cdot \nabla$

$895 \div 5$

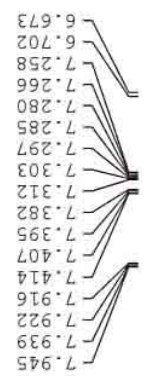

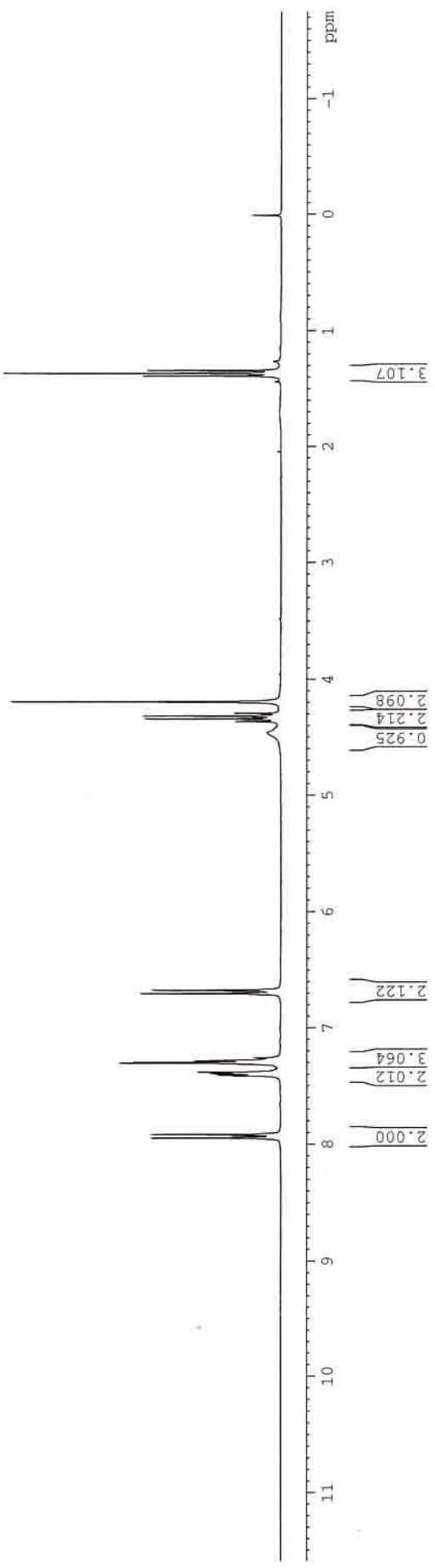

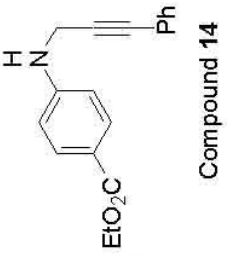



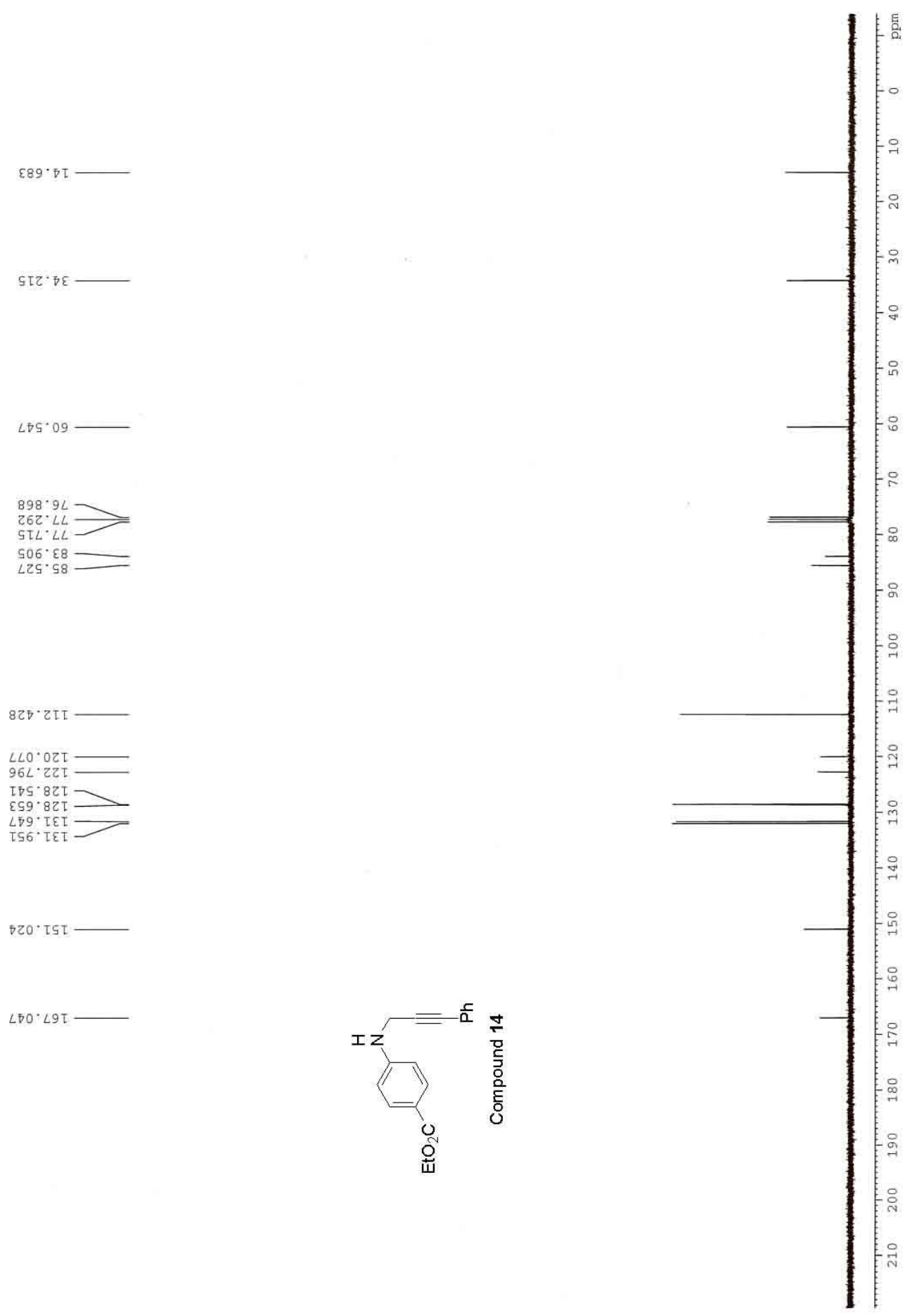
$200 \cdot 0-\longrightarrow$
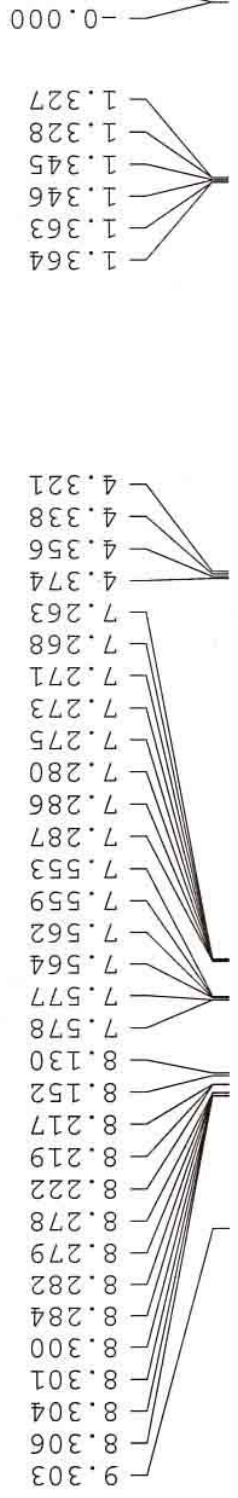

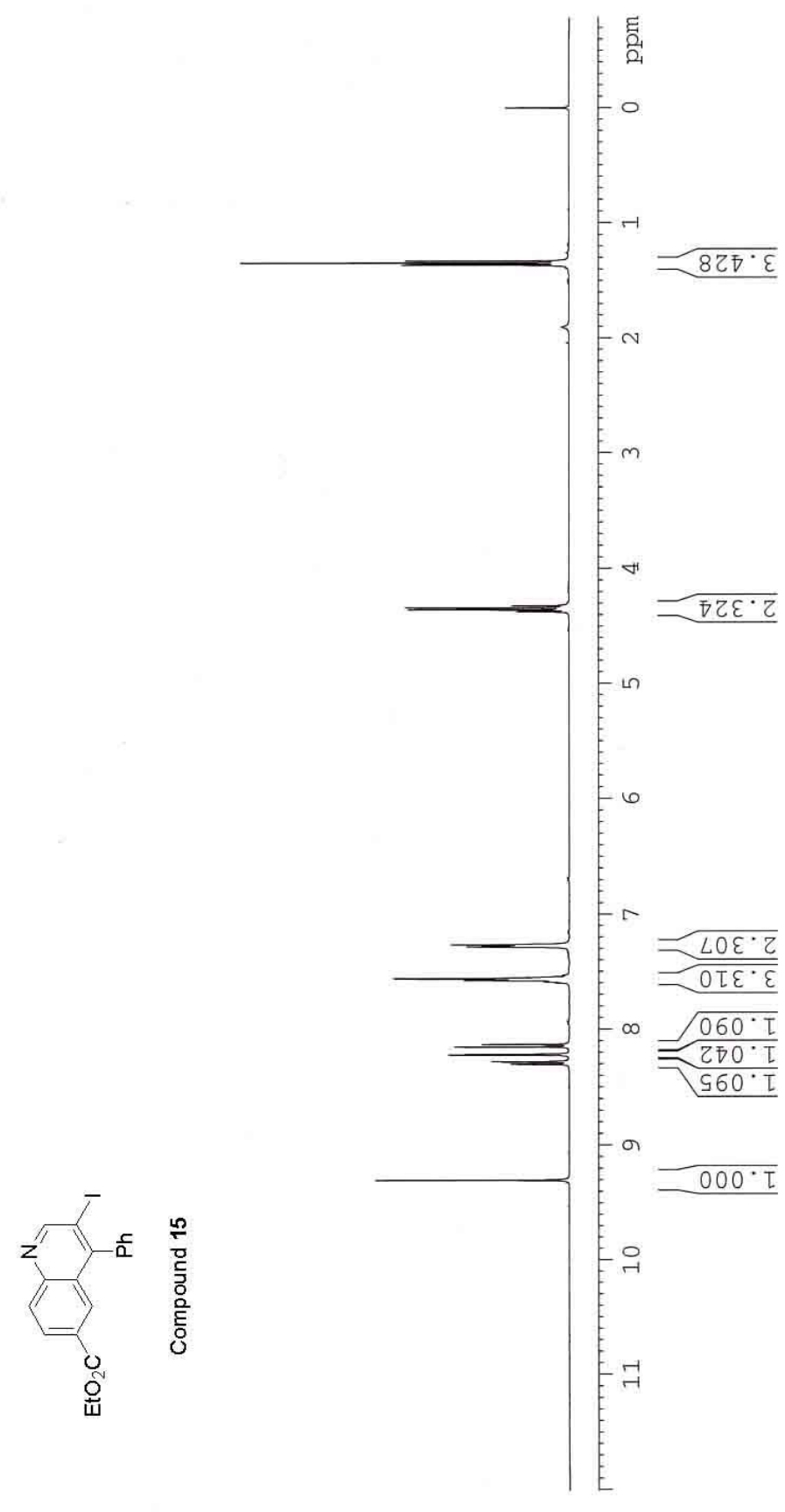


$8 L Z \cdot 7 T$

$$
\text { 9ธฑเป9 }
$$

S $8 L \cdot 9 L$

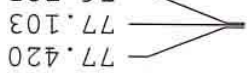

$8 L Z \cdot L 6$

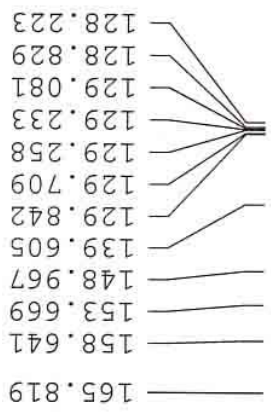

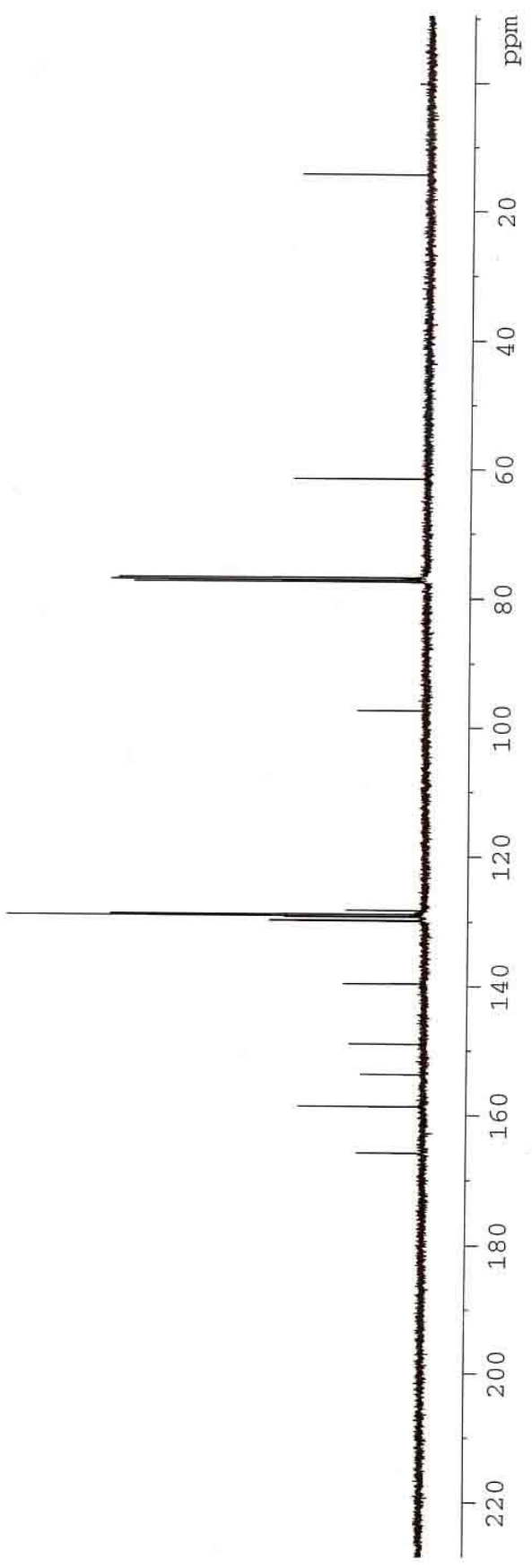

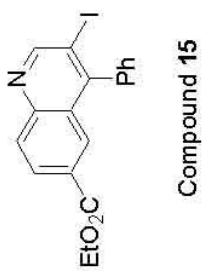



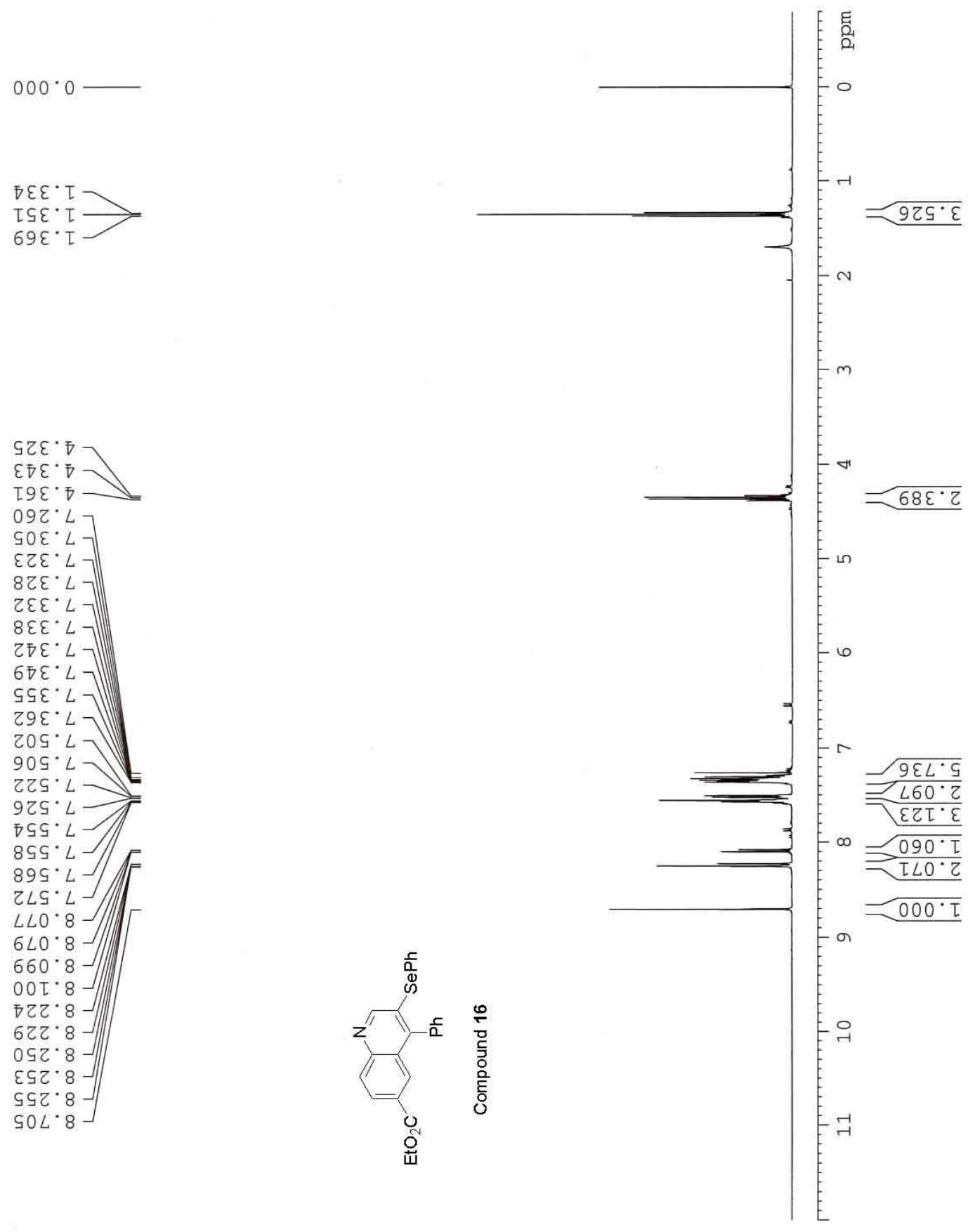


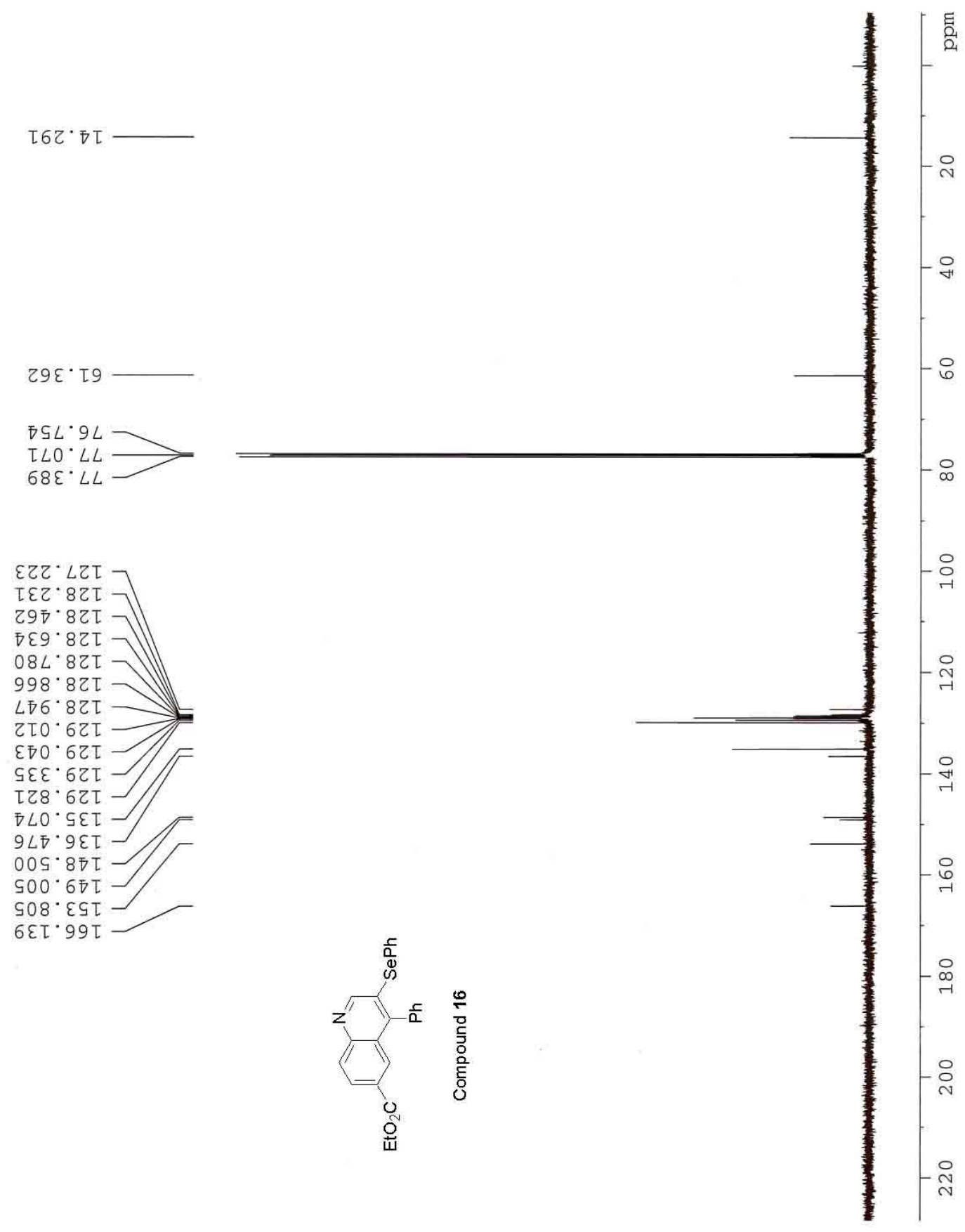



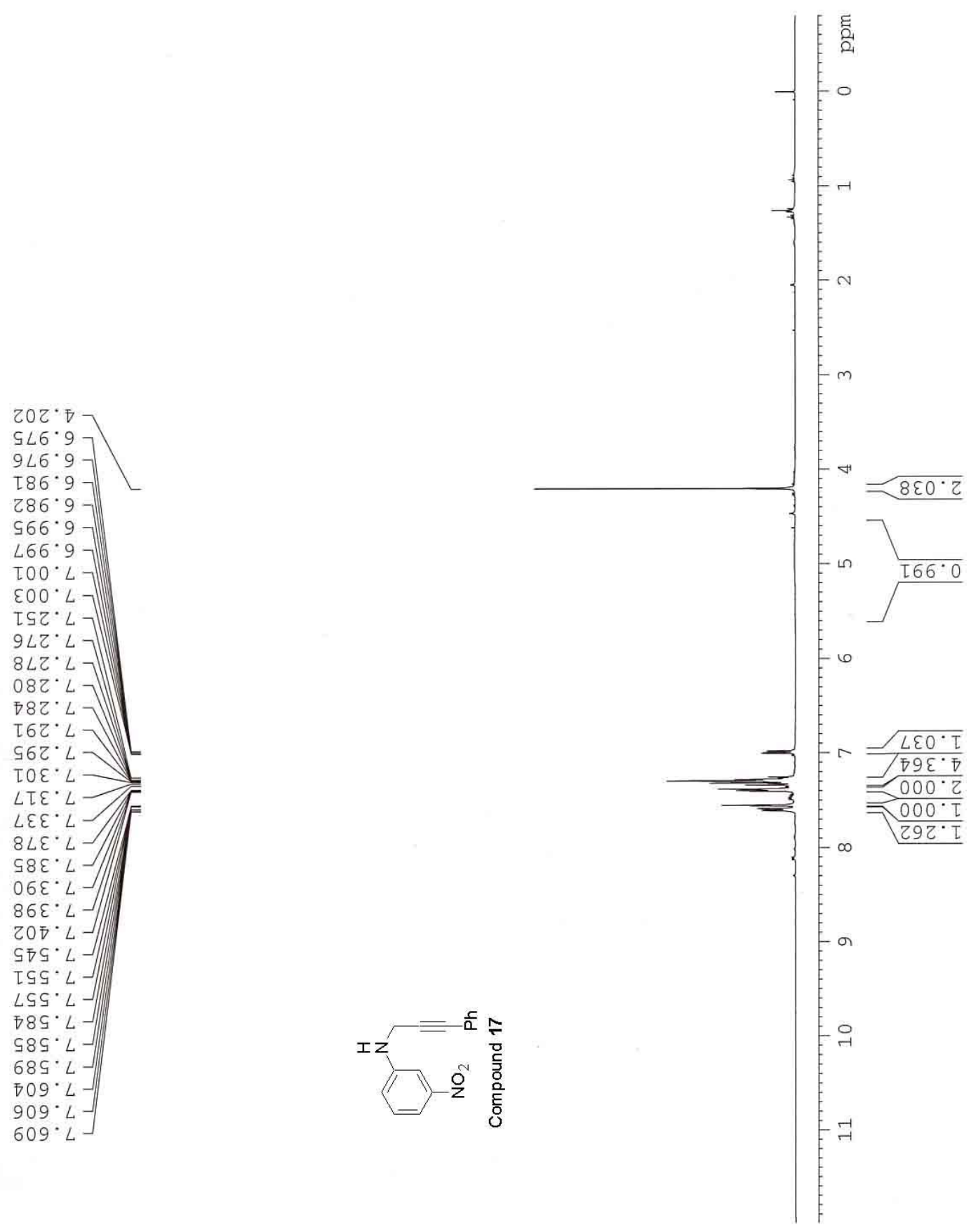

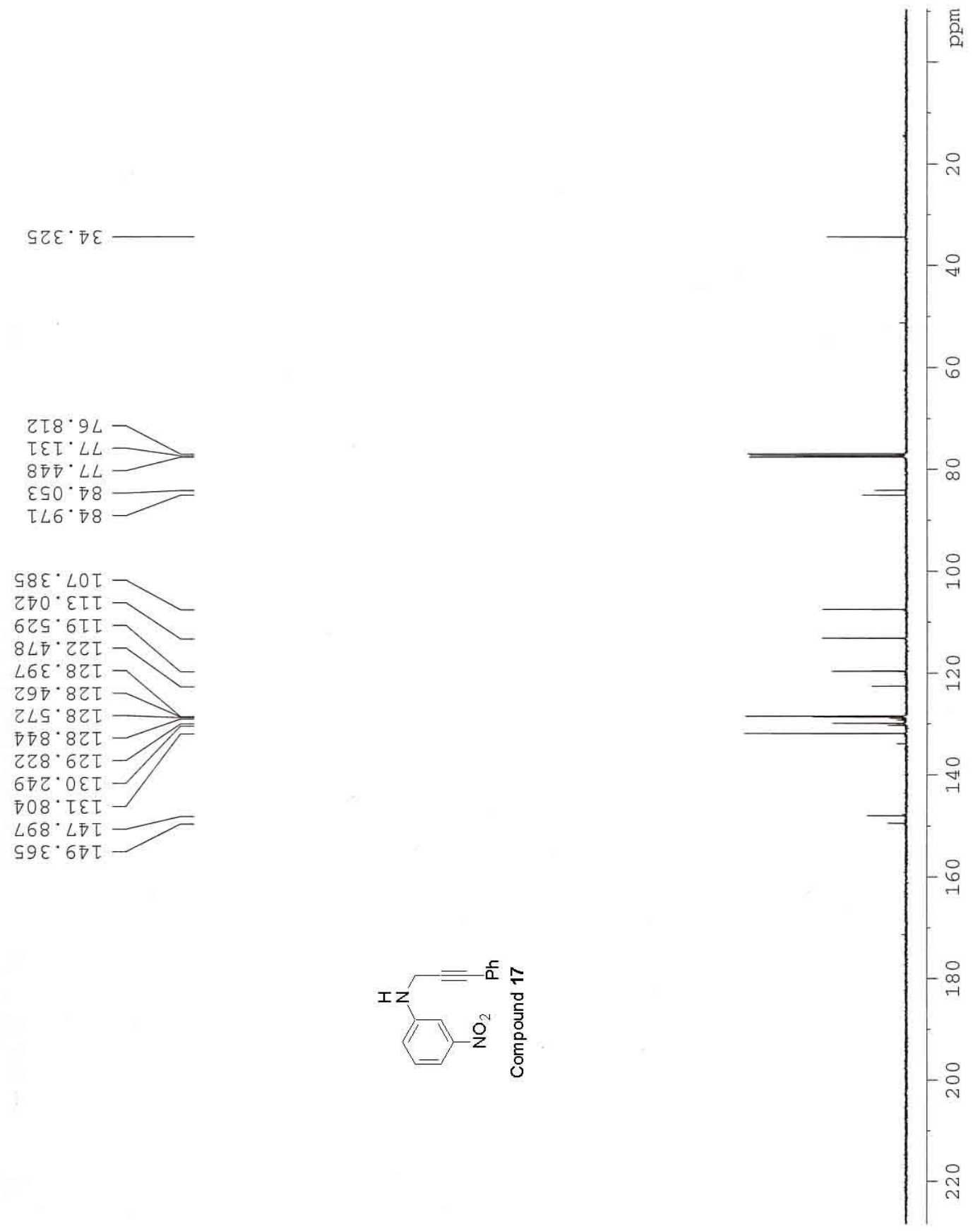

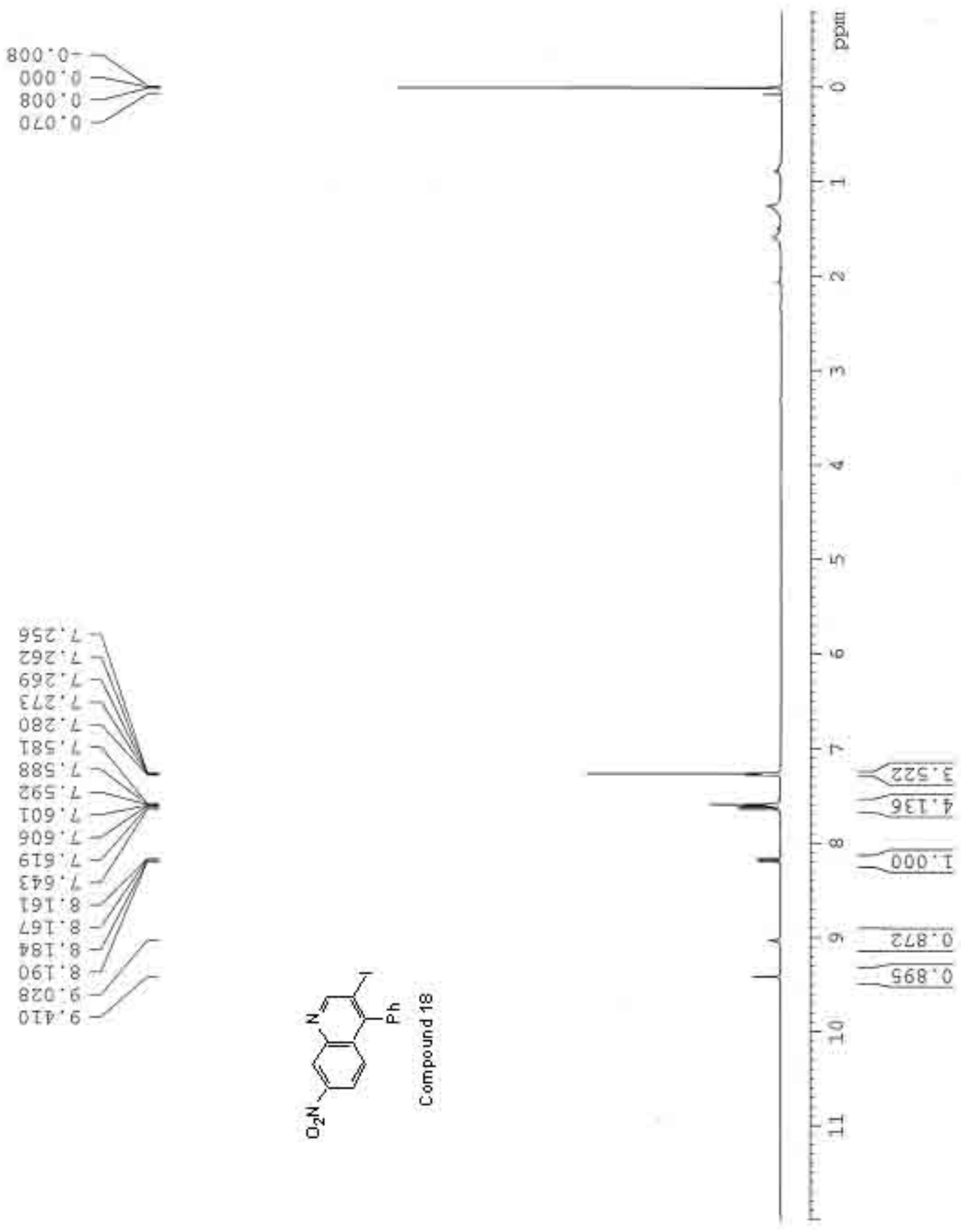


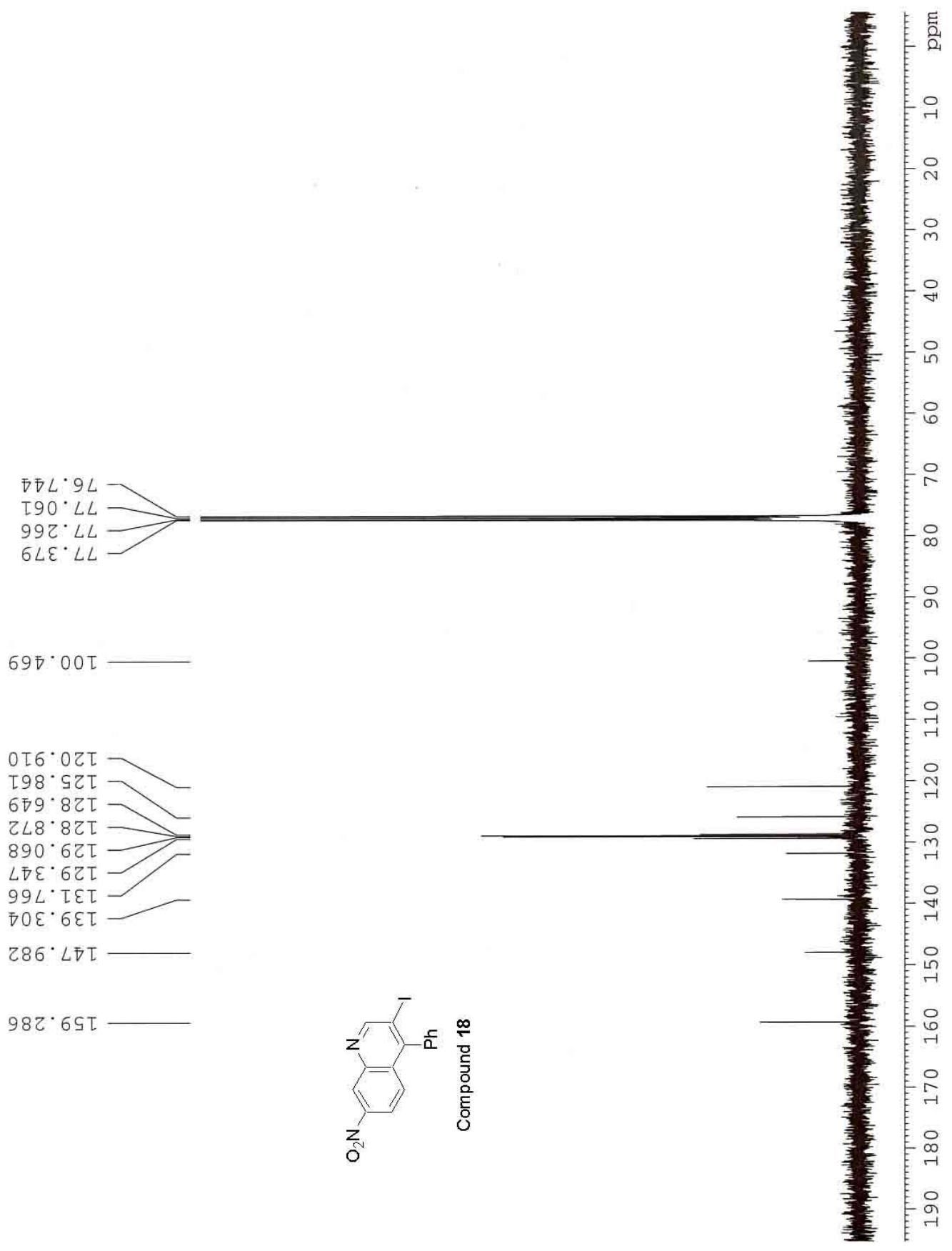


$220 \cdot 0-$
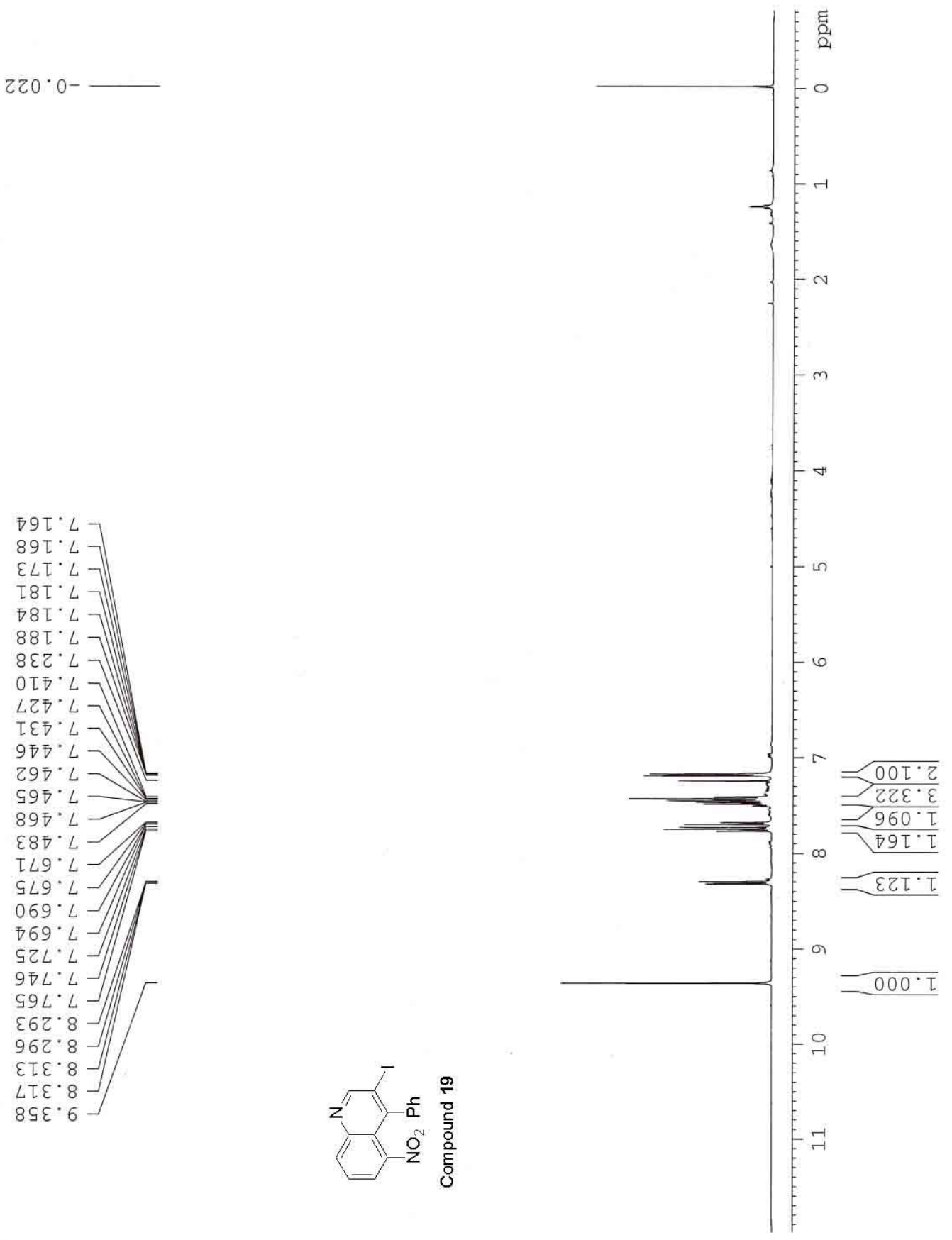


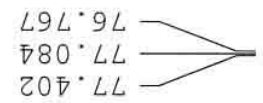

てカர・ZOL

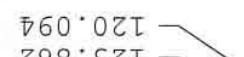
\begin{tabular}{l}
$\mathrm{\nabla} Z 8^{\circ} \cdot \varepsilon \tau \tau$ \\
\hline
\end{tabular} $992 \cdot 82 \mathrm{\longrightarrow}$ † $\cdot 6 Z I$ $\varepsilon 9 \nabla^{\circ} 6 Z \tau$ $8 Z \tau \cdot \nabla \varepsilon L$ Ф乙币. $6 \varepsilon \tau$ $866^{\circ} \angle \nabla T$ $\angle 96^{\circ} 8 \mathrm{DT}$

29.'89I

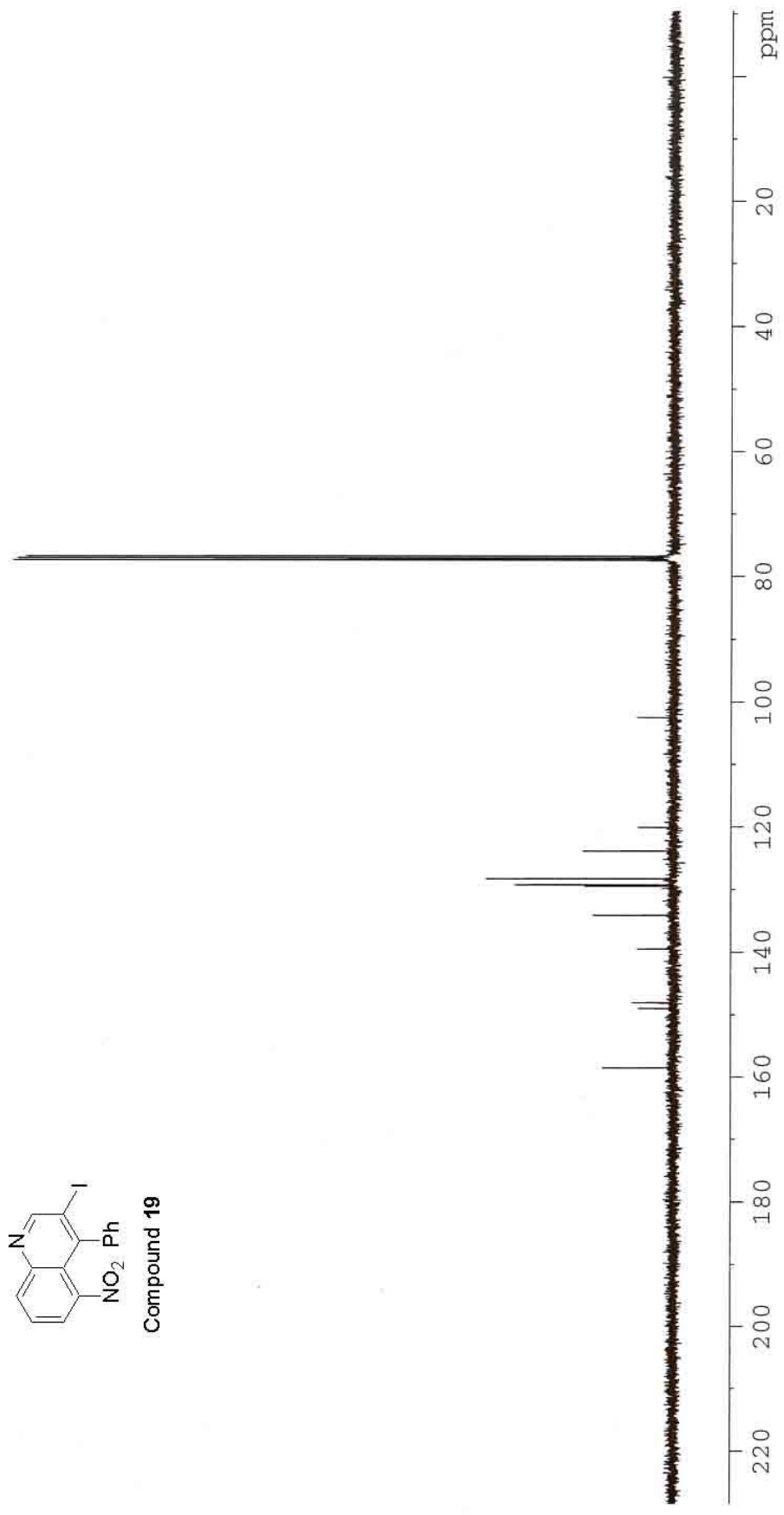


$\varepsilon 20^{\circ} \nabla=$

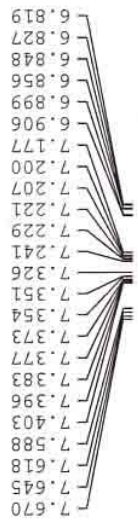

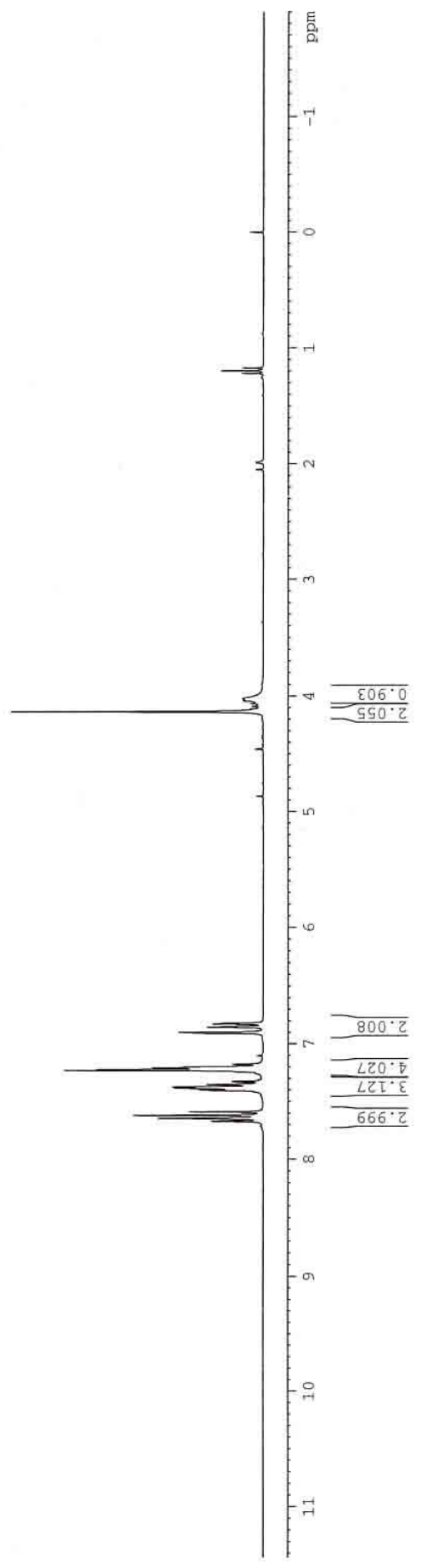

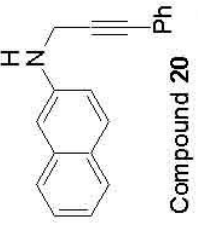



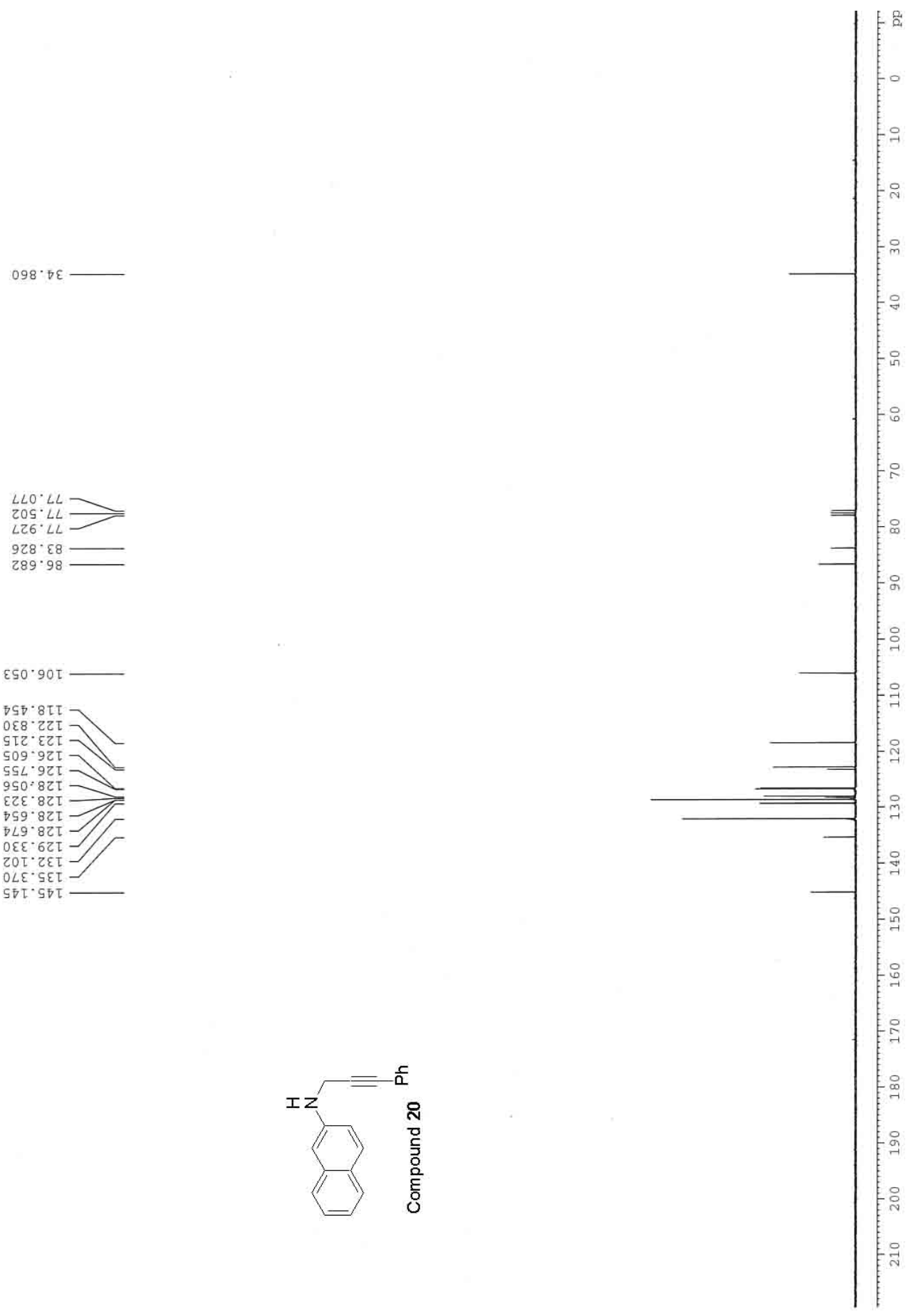

ำ

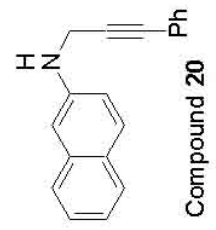




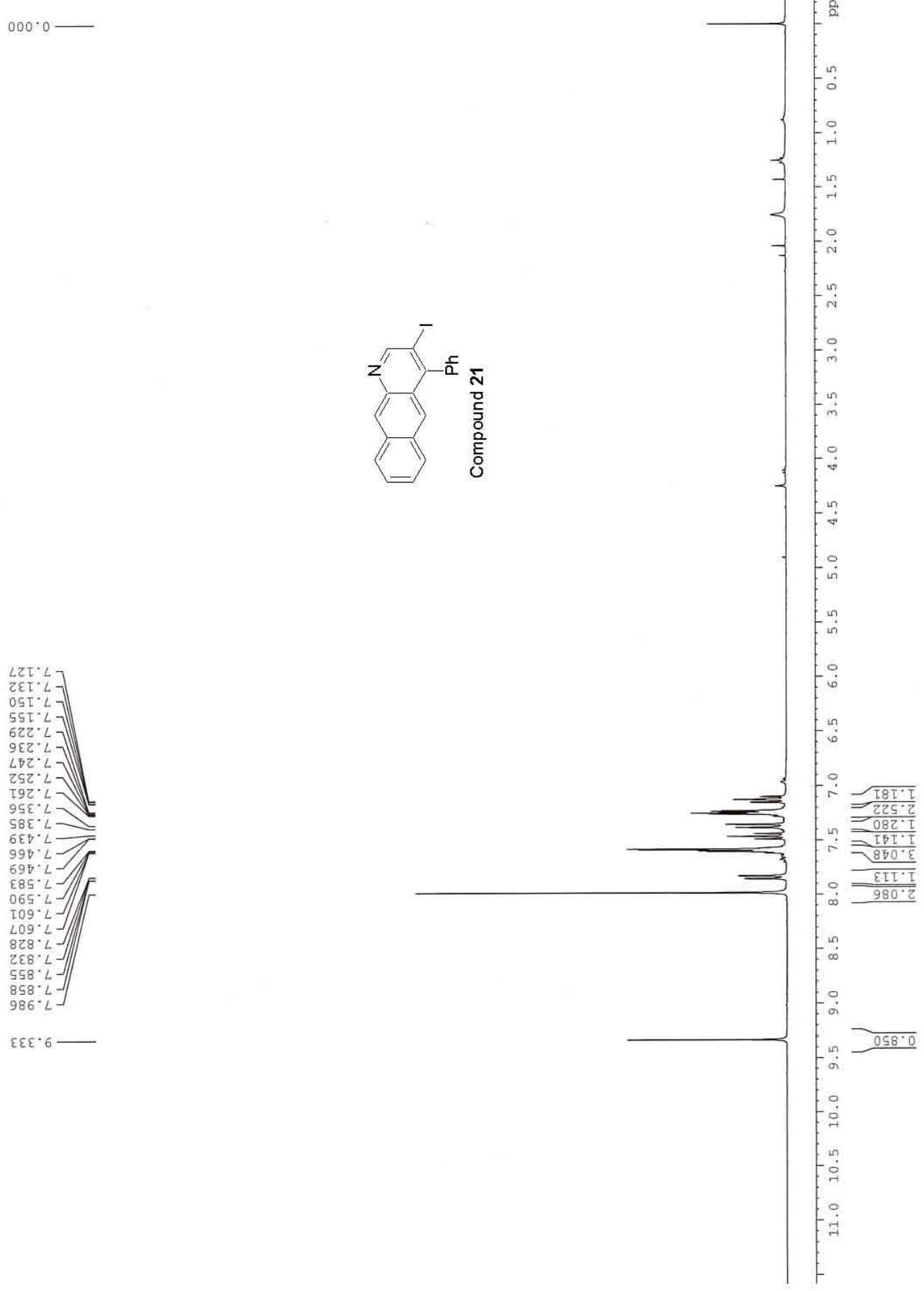




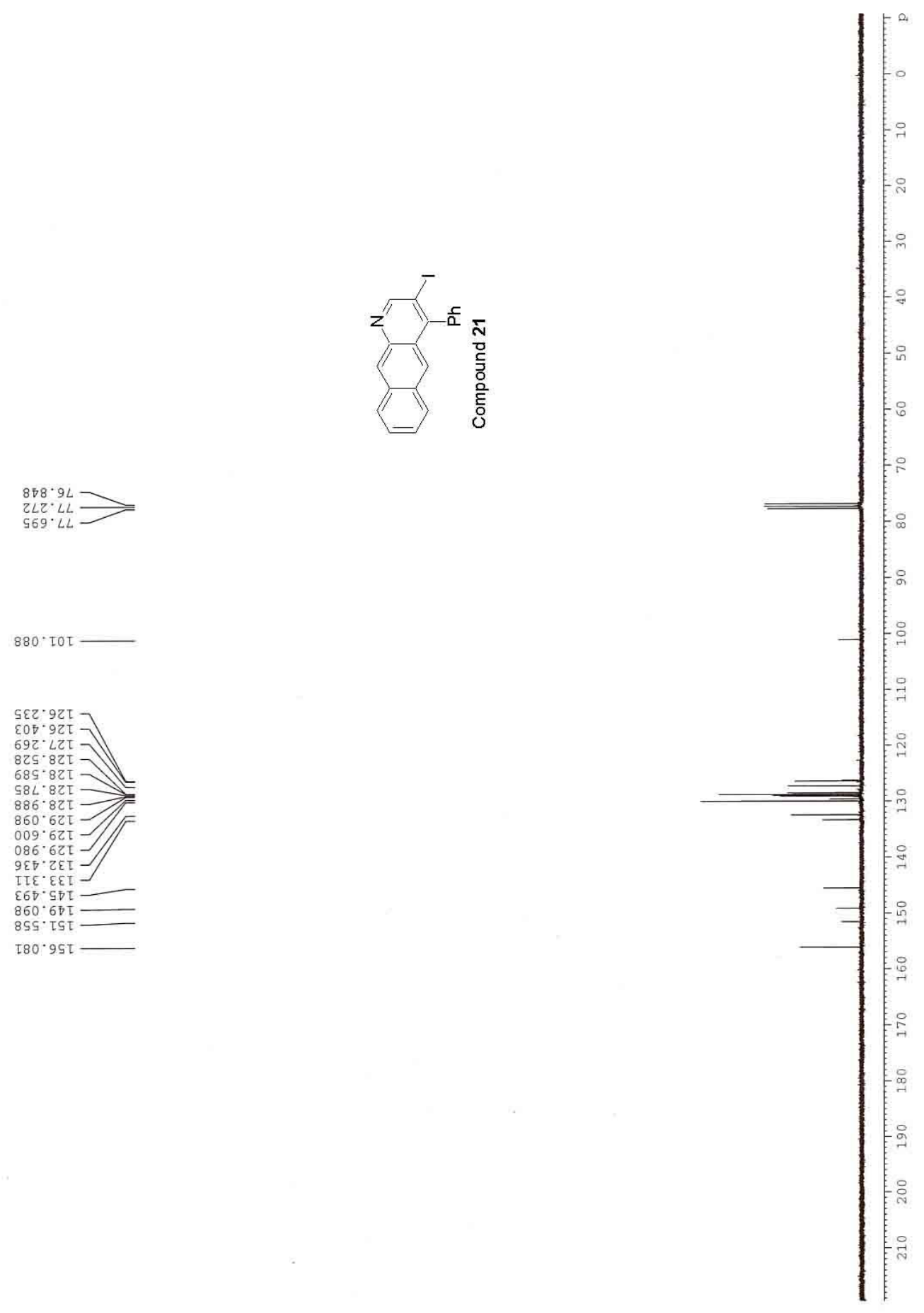




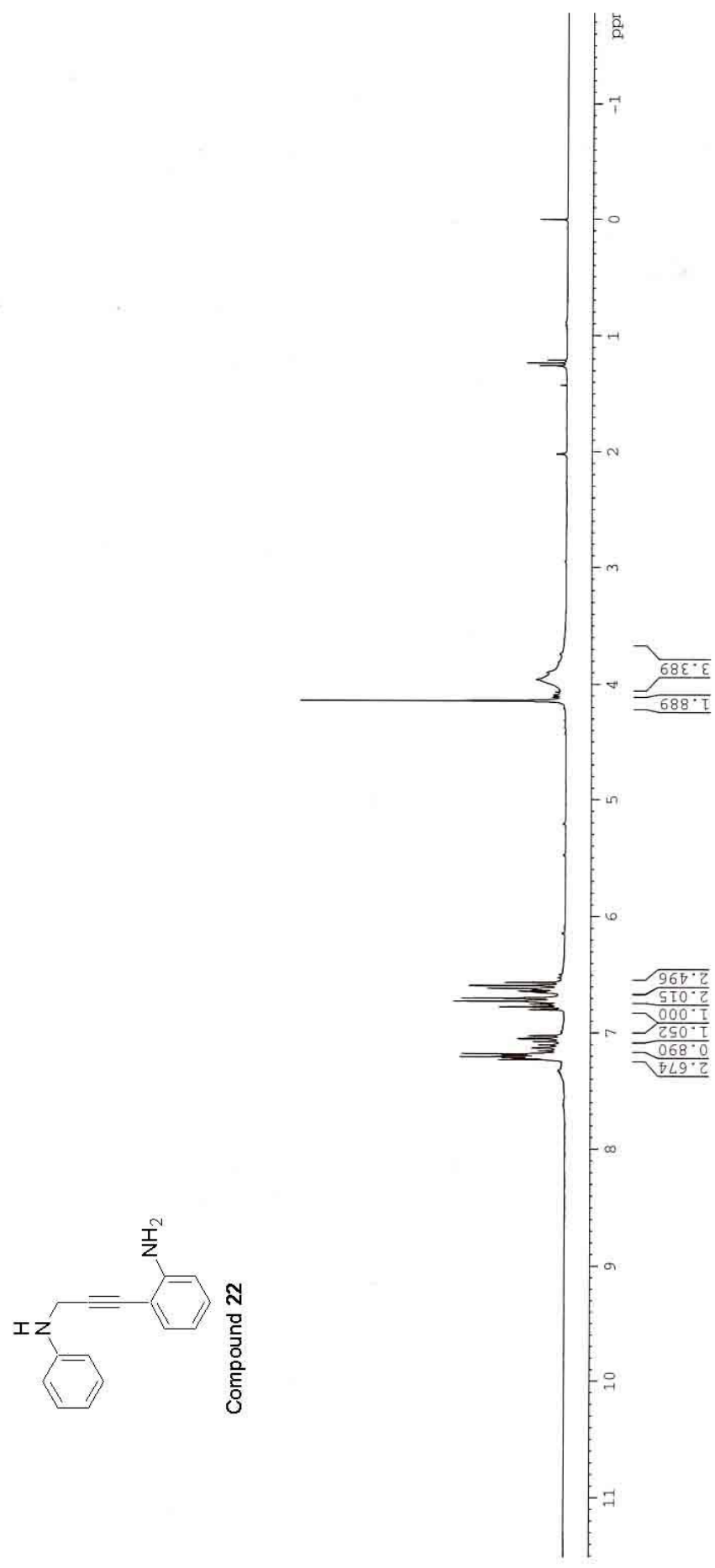



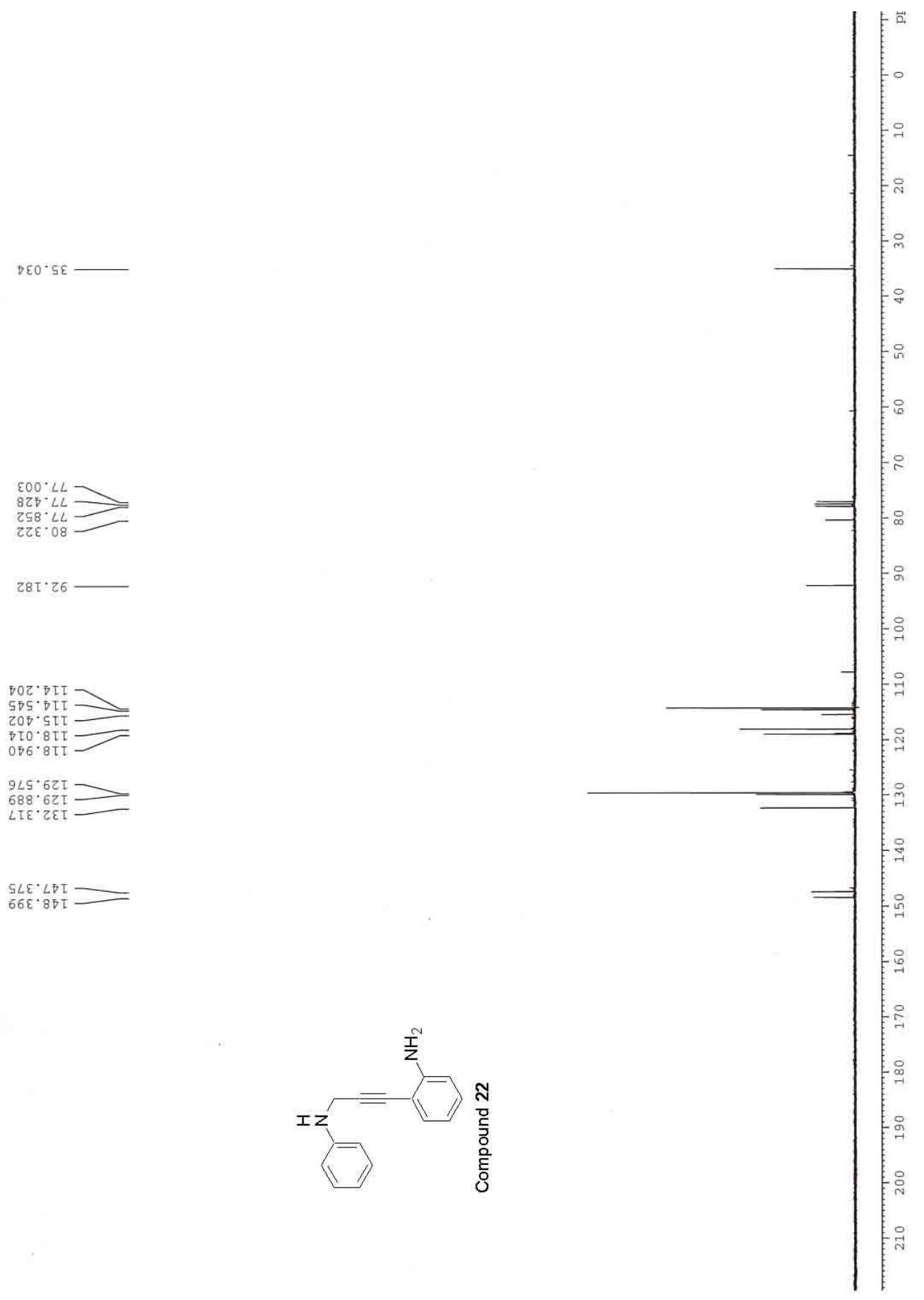

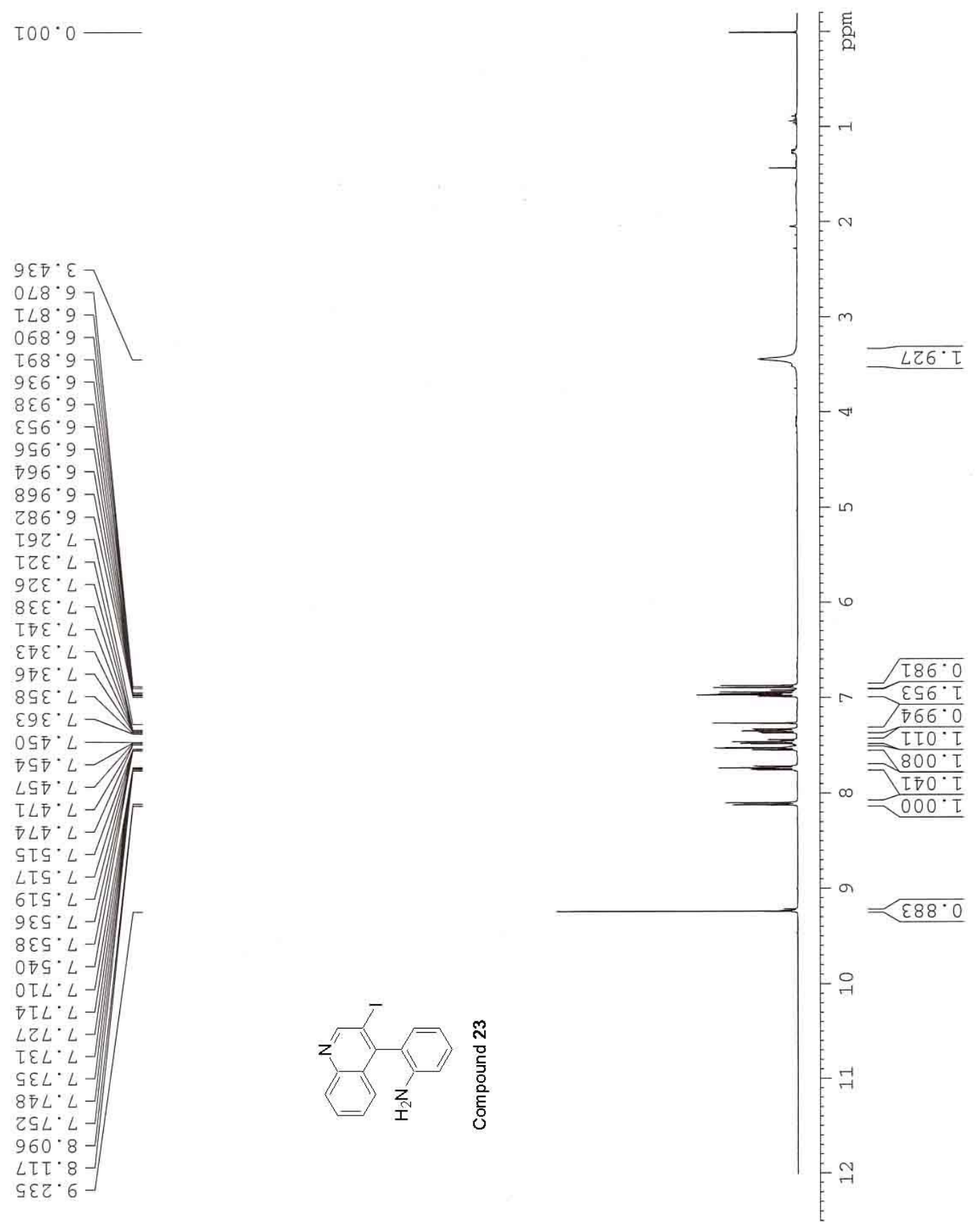

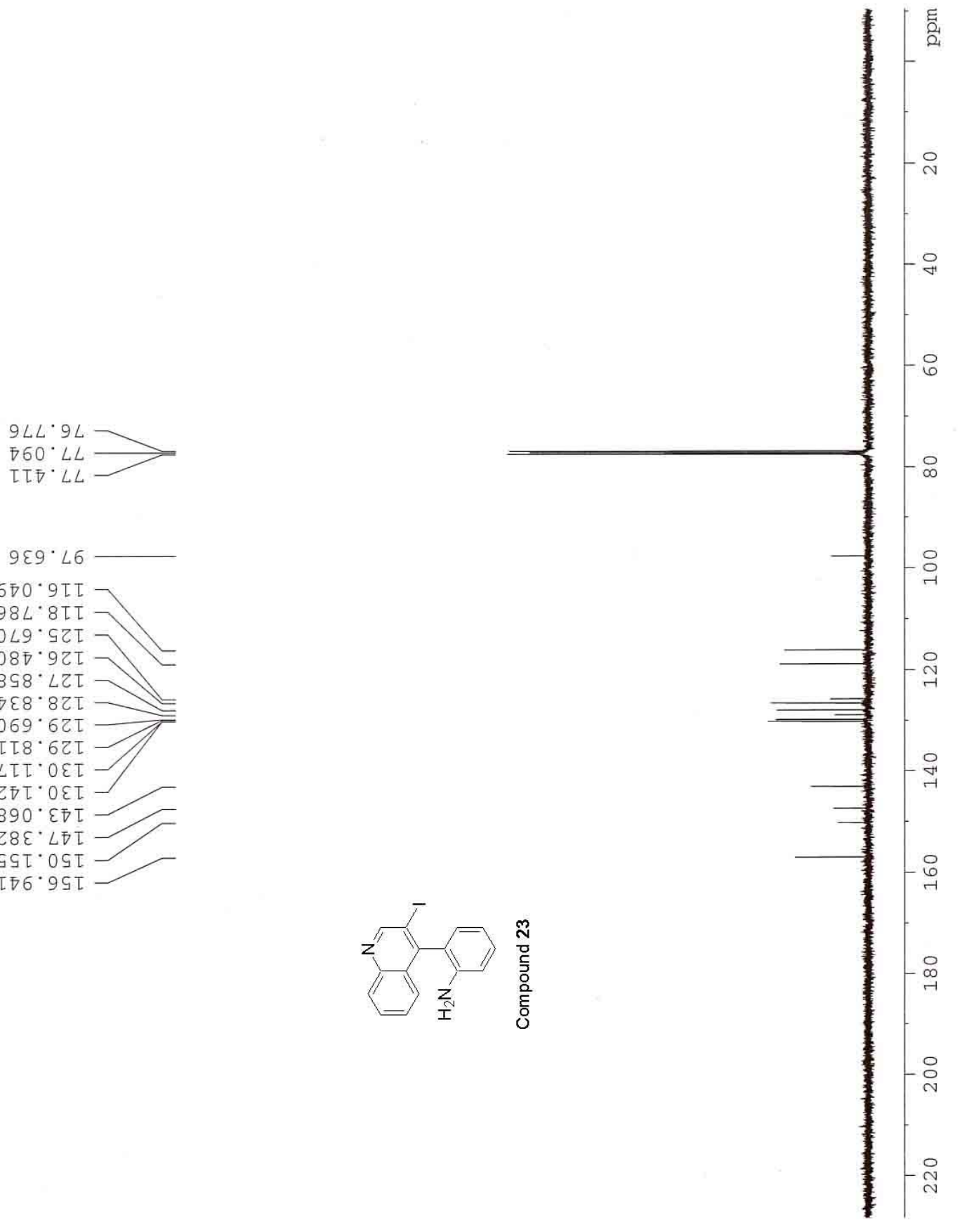
$800 \cdot 0-\square$

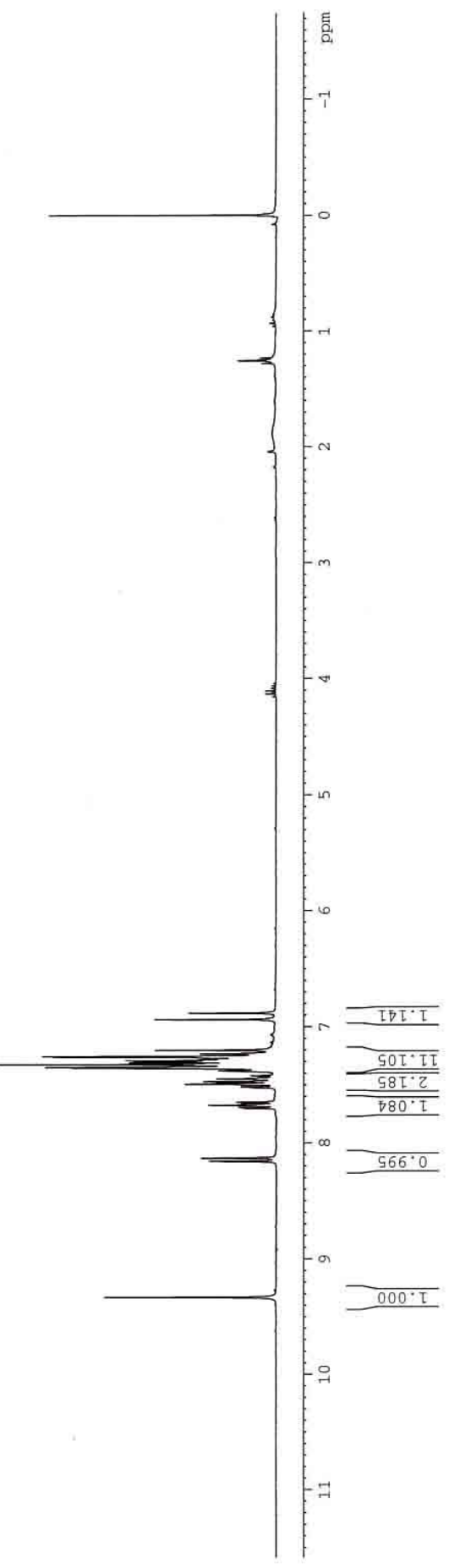



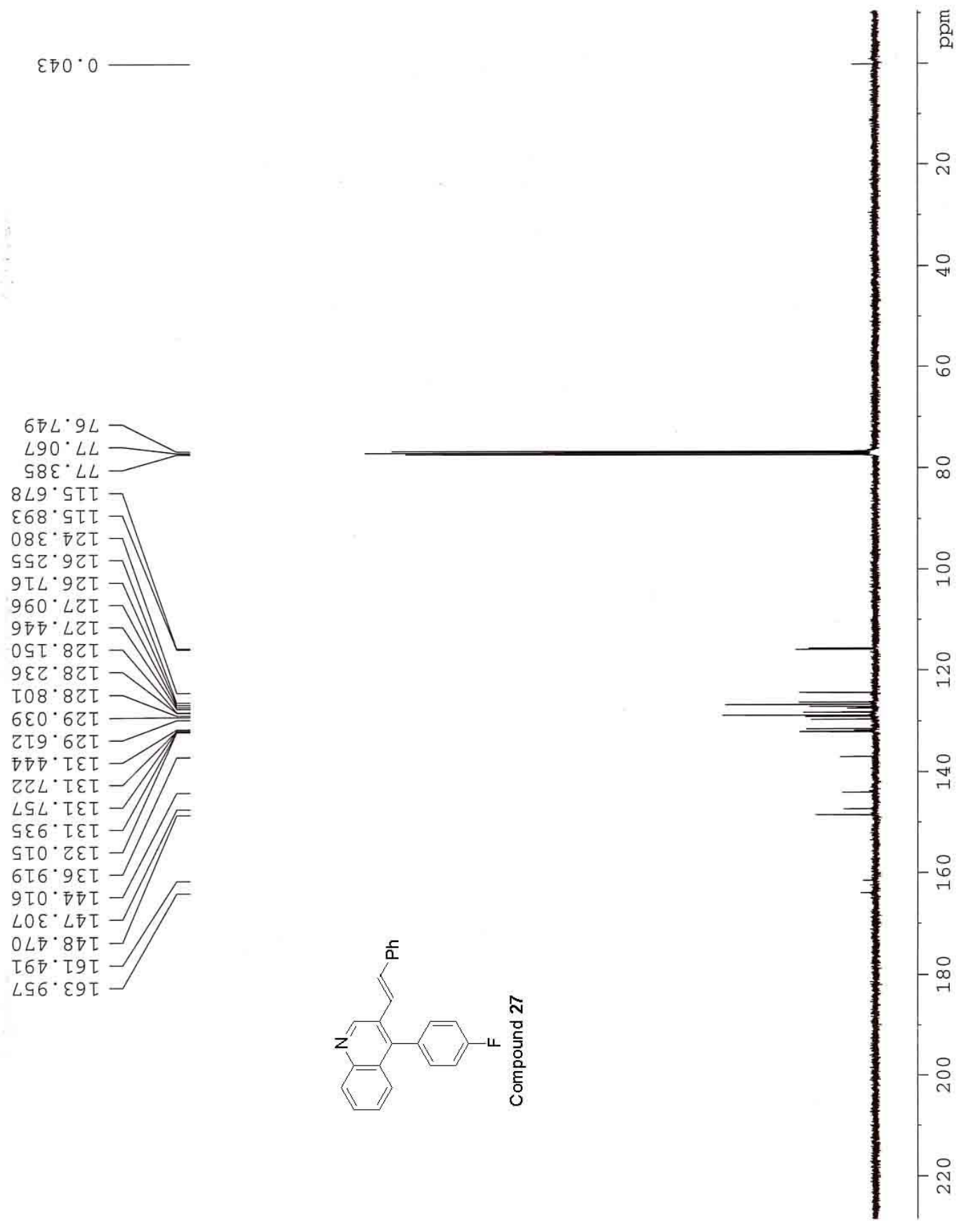

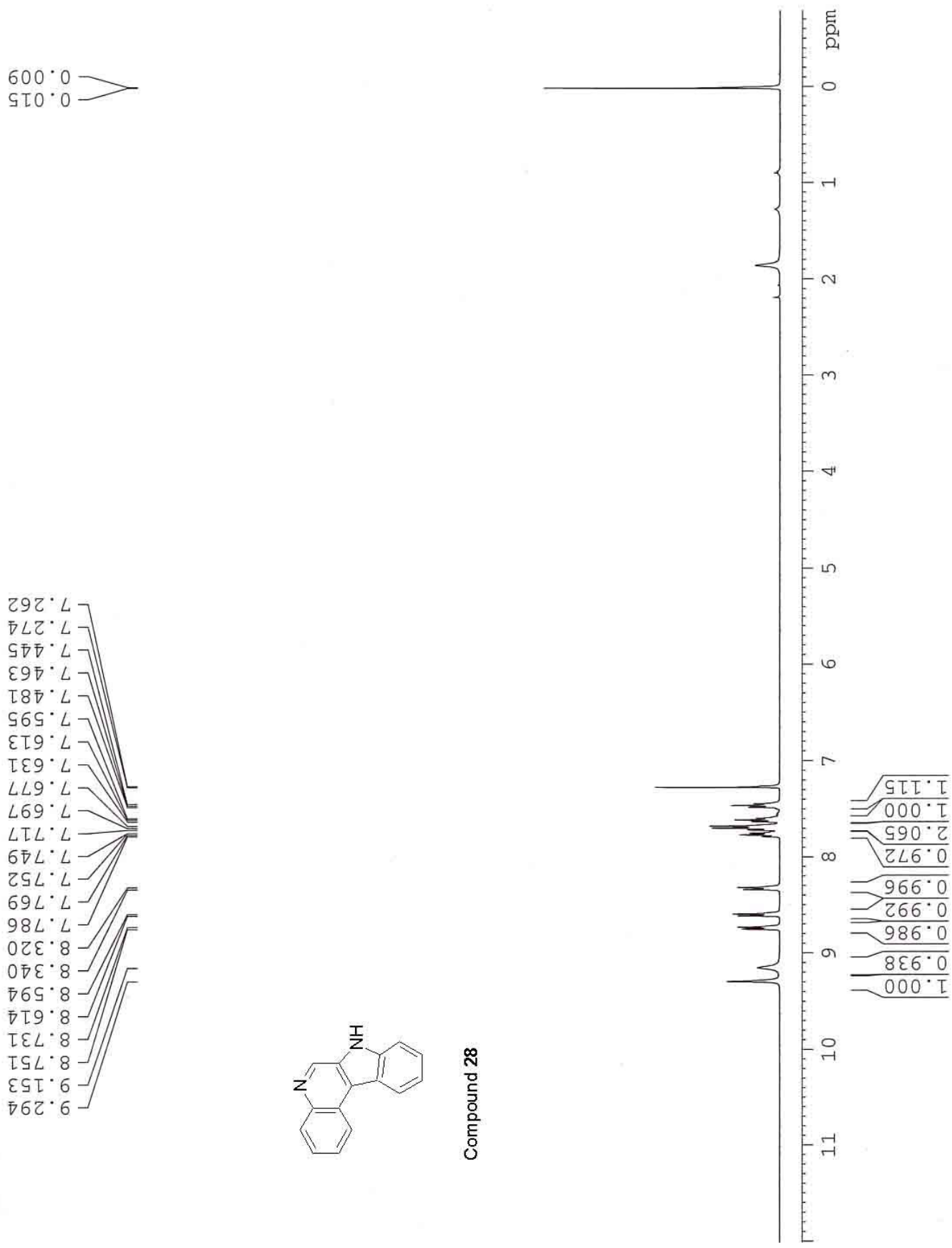

in

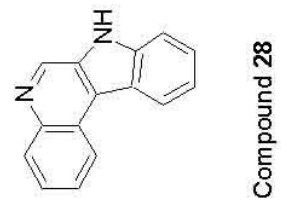




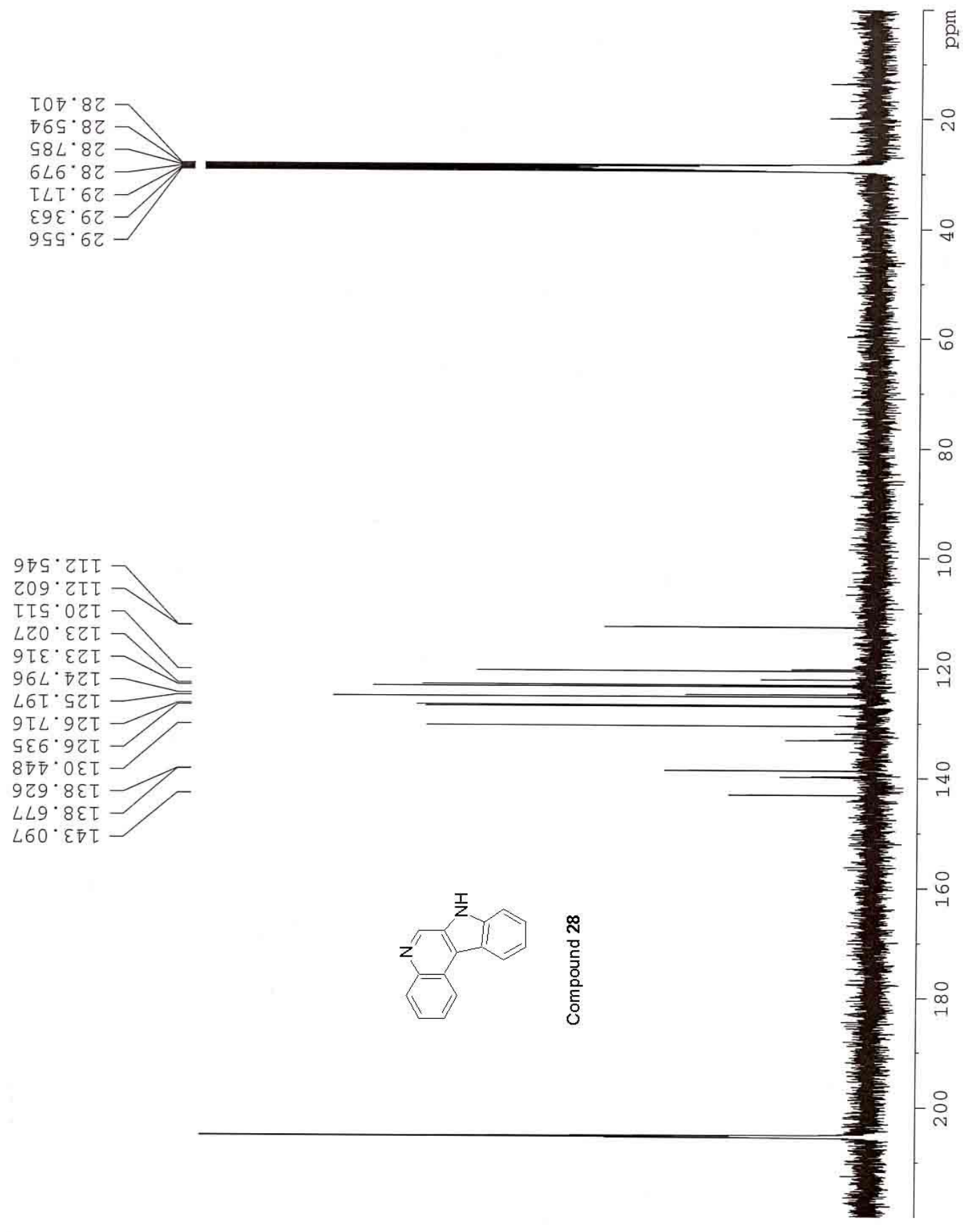

